\title{
CFHTLenS: Galaxy bias as function of scale, stellar mass, and colour ${ }^{\star}$
}

\section{Conflicts with predictions by semi-analytic models}

\author{
Patrick Simon ${ }^{1}$ and Stefan Hilbert ${ }^{2,3}$ \\ 1 Argelander-Institut für Astronomie, Universität Bonn, Auf dem Hügel 71, 53121 Bonn, Germany \\ e-mail: psimon@astro.uni-bonn.de \\ 2 Faculty of Physics, Ludwig-Maximilians University, Scheinerstr. 1, 81679 München, Germany \\ 3 Excellence Cluster Universe, Boltzmannstr. 2, 85748 Garching, Germany
}

Received 8 April 2020 / Accepted 17 November 2020

\begin{abstract}
Galaxy models predict a tight relation between the clustering of galaxies and dark matter on cosmological scales, but predictions differ notably in the details. We used this opportunity and tested two semi-analytic models by the Munich and Durham groups with data from the Canada-France-Hawaii Telescope Lensing Survey (CFHTLenS). For the test we measured the scale-dependent galaxy bias factor $b(k)$ and correlation factor $r(k)$ from linear to non-linear scales of $k \approx 10 \mathrm{~h} \mathrm{Mpc}^{-1}$ at two redshifts $\bar{z}=0.35,0.51$ for galaxies with stellar mass between $5 \times 10^{9}$ and $3 \times 10^{11} h_{70}^{-2} \mathrm{M}_{\odot}$. Our improved gravitational lensing technique accounts for the intrinsic alignment of sources and the magnification of lens galaxies for better constraints for the galaxy-matter correlation $r(k)$. Galaxy bias in CFHTLenS increases with $k$ and stellar mass; it is colour-dependent, revealing the individual footprints of galaxy types. Despite a reasonable model agreement for the relative change with both scale and galaxy properties, there is a clear conflict for $b(k)$ with no model preference: the model galaxies are too weakly clustered. This may flag a model problem at $z \gtrsim 0.3$ for all stellar masses. As in the models, however, there is a high correlation $r(k)$ between matter and galaxy density on all scales, and galaxy bias is typically consistent with a deterministic bias on linear scales. Only our blue and low-mass galaxies of about $7 \times 10^{9} h_{70}^{-2} \mathrm{M}_{\odot}$ at $\bar{z}=0.51$ show, contrary to the models, a weak tendency towards a stochastic bias on linear scales where $r_{\mathrm{ls}}=0.75 \pm 0.14$ (stat.) \pm 0.06 (sys.). This result is of interest for cosmological probes, such as $E_{\mathrm{G}}$, that rely on a deterministic galaxy bias. We provide Monte Carlo realisations of posterior constraints for $b(k)$ and $r(k)$ in CFHTLenS for every galaxy sample in this paper at the CDS.
\end{abstract}

Key words. gravitational lensing: weak - cosmology: observations - large-scale structure of Universe - galaxies: evolution

\section{Introduction}

The broad diversity of galaxy types and their large-scale clustering requires complex and numerically challenging physics that in models is approximated or empirically calibrated. This leaves us today with a variety of modelling techniques that produce a range of theoretical, not always consistent predictions for galaxy statistics (e.g. Vogelsberger et al. 2020; Springel et al. 2018; Lacey et al. 2016; Henriques et al. 2017; Campbell et al. 2018; Saghiha et al. 2017). Of major interest for model tests, due to the key role of dark matter halos for galaxy physics, is the predicted relative clustering of matter and galaxies: the galaxy bias.

Galaxy bias varies with galaxy type (Mo et al. 2010, and references therein), rendering it a type-dependent footprint for the galaxy-matter connection. Motivated by this idea, as a test we compare measurements of these footprints in the CanadaFrance-Hawaii Lensing Survey (CFHTLenS) data (Erben et al. 2013) to predictions by two semi-analytic models (SAMs) for various galaxy types, redshifts, and spatial scales from the linear down to the highly non-linear regime at $k \approx 10 \mathrm{hpc}^{-1}$.

\footnotetext{
* Galaxy bias and galaxy redshift distribution functions are only available at the CDS via anonymous ftp to cdsarc.u-strasbg.fr (130.79.128.5) or via http://cdsarc.u-strasbg.fr/viz-bin/ cat $/ \mathrm{J} / \mathrm{A}+\mathrm{A} / 646 / \mathrm{A} 71$
}

They are the Munich model 'H15' by Henriques et al. (2015) and the Durham model 'L12' by Lagos et al. (2012). These SAMs are different implementations of a technique that populates dark matter halos in cosmological simulations, here the Millennium Simulation (Springel et al. 2005), with model galaxies by emulating known galaxy physics (Cole et al. 2000, for a general overview). Their predictions for the excess surface matter-density around galaxy pairs were already studied in Saghiha et al. (2017) and Simon et al. (2019). Here we focus on galaxy biasing in these SAMs.

Among the many ways to define galaxy bias (see e.g. Desjacques et al. 2018), our model tests employ the definition by Tegmark \& Bromley (1999) in terms of galaxy and matter power spectra for the (comoving) Fourier modes $k$,

$$
b(k):=\sqrt{\frac{P_{\mathrm{g}}(k)}{P_{\mathrm{m}}(k)}} ; \quad r(k):=\frac{P_{\mathrm{gm}}(k)}{\sqrt{P_{\mathrm{m}}(k) P_{\mathrm{g}}(k)}} .
$$

These two biasing functions are measured for a specific galaxy population and redshift. They incorporate the power spectra of fluctuations in the matter density field, $P_{\mathrm{m}}(k)$; in the galaxy density field, $P_{\mathrm{g}}(k)$; and their cross power $P_{\mathrm{gm}}(k)$. In the definition of $P_{\mathrm{g}}(k)$, a Poisson sampling shot-noise is subtracted, which may produce $r(k)>1$ (Peebles 1980; Guzik \& Seljak 2001). The Eq. (1) are sensitive to a stochastic bias; however, restricted 
to two-point statistics, they do not discriminate between a non-linear deterministic bias and a stochastic bias. The only exceptions are linear scales where $k \ll 1 h \mathrm{Mpc}^{-1}$ and density fluctuations $\tilde{\delta}(\boldsymbol{k})$ are small (Somerville et al. 2001; Dekel \& Lahav 1999). For example, on linear scales $r(k)<$ 1 indicates a stochastic scatter $\epsilon_{k}$ in the relation $\tilde{\delta}_{\mathrm{g}}(\boldsymbol{k})=$ $b(k) \tilde{\delta}_{\mathrm{m}}(\boldsymbol{k})+\epsilon_{k}$ between galaxy density contrast, $\tilde{\delta}_{\mathrm{g}}$, and matter density contrast, $\tilde{\delta}_{\mathrm{m}}$, whereas for large density fluctuations $r(k)<1$ can also indicate $\tilde{\delta}_{\mathrm{g}}(\boldsymbol{k})=b_{k}\left[\tilde{\delta}_{\mathrm{m}}(\boldsymbol{k})\right]+\epsilon_{k}$ with a nonlinear mapping $b_{k}[x]$ and smaller (or vanishing) $\epsilon_{k}$. Studies of galaxy clustering show that both non-linearity and stochasticity increase towards smaller scales, and galaxy bias is essentially linear and deterministic $\left(\epsilon_{k}=0\right)$ at $2 \pi k^{-1} \gtrsim 30 h^{-1} \mathrm{Mpc}$ (e.g. Friedrich et al. 2018; Gruen et al. 2018; Dvornik et al. 2018; Di Porto et al. 2016; Patej \& Eisenstein 2016; Kovač et al. 2011; Swanson et al. 2008; Wild et al. 2005; Tegmark \& Bromley 1999). Despite the limitations, these measures of galaxy biasing highlight differences between galaxy populations and their relation to the matter density field, they are easily measured in simulations (Springel et al. 2018; Weinberg et al. 2004), and are hence a useful quantity to test galaxy models.

Perfectly suited to measuring scale-dependent galaxy bias is the analysis of the weak gravitational-lensing effect (Schneider et al. 2006). This exploits small coherent distortions of distant galaxy images (sources) by the large-scale matterdensity field in the foreground. For second-order statistics the analysis correlates shear distortions with lens galaxy positions to obtain, apart from an amplitude normalisation, galaxy bias in projection. This can be normalised or deprojected if the redshift distributions of sources and lens galaxies, as well as the background cosmology, mainly $\Omega_{\mathrm{m}}$, is known (van Waerbeke 1998; Schneider 1998; Hoekstra et al. 2001). Several previous studies have successfully applied this (or a similar) lensing analysis (Dvornik et al. 2018; Prat et al. 2018; Comparat et al. 2013; Jullo et al. 2012; Simon et al. 2007; Pen et al. 2003; Hoekstra et al. 2001), but they neglect the effects of lensing magnification and source intrinsic alignments (e.g. Hirata \& Seljak 2004; Unruh et al. 2020). In particular, intrinsic alignments (IA) have to be included in the lensing signal to reach an accuracy of several per cent for $b(k)$ and $r(k)$, as demonstrated in Simon \& Hilbert (2018, hereafter SH18) by using mock galaxy and shear catalogues that are similar to ours here. We apply the SH18 procedure with some improvements to measure the functions in Eq. (1).

This lensing technique probes galaxy biasing with a minimum of assumptions and is also valid in the non-linear regime. This sets it apart from other techniques that, for instance, only apply in the weakly linear regime; require a theoretical reference model for the clustering of matter as a function of scale; or depend on a detailed physical model for the clustering of matter and galaxies (e.g. Cacciato et al. 2013; Marulli et al. 2013; Dvornik et al. 2018; Heymans et al. 2021). However, our technique employs flexible model functions for $b(k)$ and $r(k)$ to stabilise the deprojection of the projected galaxy bias. Although based on a halo model prescription, their purpose notably is not an accurate interpretation of the projected galaxy bias in terms of a physical model, but rather an accurate deprojection of the model-free biasing functions. The completely generic templates in SH18 could be used equally well, albeit with less accuracy. To highlight our focus on the biasing functions, we refer to the stabilising model functions in the reconstruction as 'templates'.

The outline of this paper is as follows. Section 2 describes our CFHTLenS shear catalogue and the selection of galaxy (foreground) samples for the analysis. For these we estimate the redshift distributions (lenses and sources) and the mean number density of the lenses; both are used in the analysis. For the model predictions, we select similar galaxy samples from the SAMs in Sect. 3, accounting for the stellar-mass errors in CFHTLenS. Section 4 summarises the template-based deprojection technique for $b(k)$ and $r(k)$. Our new analysis improves on that of SH18 with regard to the transformation bias in the aperture statistics (Sect. 4.4), the prior for the template parameters of the biasing functions (Appendix E), and an allowed mismatch between matter and satellite galaxy distribution inside dark matter halos (Sect. 4.6). We present raw (i.e. not normalised) CFHTLenS measurements for the projected galaxy bias already in this section. We compare our main results for $b(k)$ and $r(k)$ to the H15 and L12 predictions in Sect. 5, along with some physical interpretation, and discuss the results in Sect. 6.

The fiducial background cosmology defines the projection of galaxy bias and the lensing kernel; a small additional dependence is introduced by the shape of a fiducial matter power spectrum that impacts the smoothing in the projection. Our results adopt a flat $\Lambda$ cold dark matter $(\Lambda \mathrm{CDM})$ cosmology with a matter density parameter of $\Omega_{\mathrm{m}}=0.306$, a Hubble constant of $H_{0}=$ $h 100 \mathrm{~km} \mathrm{~s}^{-1} \mathrm{Mpc}^{-1}$ and $h=0.683$, a baryon matter density of $\Omega_{\mathrm{b}}=0.0479$, and a primordial spectral index of $n_{\mathrm{s}}=0.965$, in agreement with Planck Collaboration XIII (2016) (Sect. 4.1). Systematic errors in the amplitude of the CFHTLenS biasing functions due to uncertainties in the fiducial cosmological model and galaxy redshift distributions are explored in Sect. 4.9.

\section{Canada-France-Hawaii Lensing Survey}

The CFHTLenS ${ }^{1}$ is a multi-colour lensing survey based on the Wide part of the Canada-France-Hawaii Survey (CFHTLS) taken with the $2 \mathrm{k} \times 4 \mathrm{k}$ MegaPrime camera (MegaCam) between 2003 and 2008 (Erben et al. 2013). The pixel size of the camera CCD chips is 0.187 arcsec. The survey consists of four patches W1 $\left(\sim 63.8 \mathrm{deg}^{2}\right)$, W2 $\left(\sim 22.6 \mathrm{deg}^{2}\right)$, W3 ( 44.2 $\left.\mathrm{deg}^{2}\right)$, and W4 $\left(\sim 23.3 \mathrm{deg}^{2}\right)$ with $154 \mathrm{deg}^{2}$ total sky area. The patches are mosaics of $1711 \times 1 \mathrm{deg}^{2}$ MegaCam fields, observed with the five filters $u^{*} g^{\prime} r^{\prime} i^{\prime} z^{\prime}$ to the limiting AB magnitudes of $z^{\prime} \lesssim$ $23.5 \mathrm{mag}$ and between 24.5 and $25.5 \mathrm{mag}$ for the other filters $(5 \sigma$ detection limit in a 2 arcsec aperture). All filters were obtained under sub-arcsec seeing conditions of which $i^{\prime}$, used for shear estimates, has a median seeing of 0.68 arcsec. After a break of the $i^{\prime}$ filter in 2008, 33 fields were observed with the slightly different $y^{\prime}$ filter. The survey combines weak-lensing data processing with THELI (Erben et al. 2013), shear measurement with lensfit (Miller et al. 2013), and photometric redshift measurement with PSF-matched photometry (Hildebrandt et al. 2012). A full systematic-error analysis of the shear measurements in combination with the photometric redshifts is presented in Heymans et al. (2012), with additional error analyses of the photometric redshift measurements presented in Benjamin et al. (2013).

The survey data product is the object and lensing catalogue $\mathrm{v} 1.1^{2}$, for which galaxies were selected from the $i^{\prime}$-band stacks with the SExtractor software (Bertin \& Arnouts 1996). The revised photometry of these objects yields a root mean square (RMS) uncertainty of $\sigma \approx 0.01-0.03 \mathrm{mag}$ for the photometric calibration in all five bands (Hildebrandt et al. 2012). From

\footnotetext{
1 http://cfhtlens.org

2 http://www.cadc-ccda.hia-iha.nrc-cnrc.gc.ca/en/ community/CFHTLens/query.html
} 
Table 1. Effective survey area $\Omega_{\mathrm{eff}}$ for individual patches and all patches combined (mask $=0$ ).

\begin{tabular}{cccccc}
\hline & W1 & W2 & W3 & W4 & W1 to W4 \\
\hline$\Omega_{\text {eff }} / \mathrm{deg}^{2}$ & 42.42 & 11.72 & 25.43 & 12.55 & 92.12 \\
\hline
\end{tabular}

Notes. Only 129 MegaCam fields that are flagged as suitable for a cosmic-shear analysis are included.

these data, photometric redshifts $z_{\mathrm{p}}$ as galaxy distance estimators were inferred with the Bayesian Photometric Redshift Code (BPZ; Benítez 2000).

From the catalogue we used only the 129 science-ready fields that are considered suitable for cosmic shear studies in Heymans et al. (2012). To estimate the effective unmasked area of this subset of fields, we counted the number of pixels in the mask files of the co-added images with mask $=0$; only source and lens galaxies from these regions are selected for the analysis. Table 1 lists the effective survey area of all patches $\mathrm{W} 1$ to $\mathrm{W} 4$, amounting in total to an effective area of $92.12 \mathrm{deg}^{2}$. We summarise here the details of our lens and source samples.

\subsection{Galaxy samples}

We measure galaxy bias for foreground galaxies that were binned in stellar mass (SM1 to SM6) or colour (RED or BLUE), and additionally in redshift (low- $z$ or high- $z$ ). Table 2 is an overview of all $2 \times 8=16$ samples and their selection criteria.

All samples select only bright objects $\left(17.5 \leq i^{\prime}<22.5\right)$ within regions of mask $=0$. At this brightness the error on the redshift estimators is typically $\sigma_{z}=(1+z) 0.04$ with a few per cent outliers. We used stellar-mass estimates in the CFHTLenS catalogue. These estimators apply stellar-synthesis modelling to the photometric data in the five bands, assuming a Chabrier (2003) initial stellar mass-function, the stellar population synthesis model by Bruzual \& Charlot (2003), the dust extinction law in Calzetti et al. (2000), and a flat $\Lambda$ CDM model with Hubble parameter of $h=0.7$ and $\Omega_{\mathrm{m}}=0.27$ for the luminosity distance, which is similar to our fiducial cosmology in Table 4. For the stellar masses, we assume the RMS error of $0.27 \mathrm{dex}$ in Velander et al. (2014).

As in SH18 we split the galaxy samples into six stellar mass bins between $5 \times 10^{9}$ and $3.2 \times 10^{11} h_{70}^{-2} \mathrm{M}_{\odot}$, and into two colour bins along the line $u^{*}-r^{\prime}=1.93 z_{\mathrm{p}}+1.85$ for the full stellar mass range. We further divided these eight samples in photometric redshift to obtain low $z$ samples with $0.2 \leq z_{\mathrm{p}}<0.44$ $(\bar{z} \approx 0.35)$ and high- $z$ samples with $0.44 \leq z_{\mathrm{p}}<0.6(\bar{z} \approx$ $0.51)$. For each sample, the total number of objects is in the range $10^{3}-10^{5}$, which corresponds to a mean number density of $0.003-0.3 \mathrm{arcmin}^{-2}$ on the sky.

\subsection{Shear catalogues}

As background sources for the lensing analysis, we use resolved galaxies with reliable photo- $z$ estimates and $i^{\prime}<2$ 24.7. Each source $i$ has an estimated $\epsilon_{i}=\epsilon_{i 1}+\mathrm{i} \epsilon_{i 2}$ gravitational shear $\gamma_{i}$ in the source line-of-sight direction and a statistical weight $w_{i}>0$, which depends on the variance of its posterior ellipticity and the ellipticity distribution in the entire source galaxy population. The additional calibration factor $m_{i}>-1$, determined with simulated CFHTLenS images, addresses the multiplicative shear bias. We accounted for the shear calibration in our analysis, equivalent to the procedure described in Miller et al. (2013), by rescaling the source ellipticities $\epsilon_{i} \rightarrow \epsilon_{i}\left(1+m_{i}\right)^{-1}$ and weights $w_{i} \rightarrow w_{i}\left(1+m_{i}\right)$. Furthermore, we subtracted from $\epsilon_{i 2}$ the additive shear bias $c_{2}$, provided by the catalogue.

We then constructed six source samples according to Table 3. For the full analysis, sources are in two samples, BACK low$z$ and BACK high- $z$, which have little redshift overlap with the low $-z$ and high- $z$ foreground samples in Sect. 2.1, but aim at a large number density of sources on the sky. For low $z$ we applied the cut $0.65 \leq z_{\mathrm{p}}<1.3$, and for high- $z$ the cut $0.75 \leq z_{\mathrm{p}}<1.3$. Additionally, for robustness tests of our results later on, we subdivided each source bin into the two complementary photo- $z$ bins BACKa and BACKb. In total, there are roughly $2 \times 10^{6}$ sources in each sample BACK low- $z$ and high- $z$, which corresponds to a number density of $6 \mathrm{arcmin}^{-2}$. However, due to the source weights, the effective source number $N_{\mathrm{eff}}:=\left(\sum_{i} w_{i}\right)^{2} / \sum_{i} w_{i}^{2}$ is roughly $20 \%$ smaller. The mean, $w$-weighted redshift of BACK low- $z$ (high- $z$ ) is $\bar{z}=0.97$ (1.03), and the weighted ellipticity dispersion (i.e. the source shape noise) in all samples is $\left\langle|\epsilon|^{2}\right\rangle^{1 / 2} \approx 0.41$, or 0.29 for each component individually.

\subsection{Redshift distributions}

The average BPZ redshift posterior of the sample galaxies is the estimated redshift distribution of a sample (which is the sum of all posteriors followed by a normalisation). According to Benjamin et al. (2013), these estimates provide a sufficiently accurate model for the actual redshift distribution $p(z)$ of CFHTLenS galaxies at $z<1.3$.

However, the BPZ posterior is binned with bin width $\Delta z=$ 0.05 and therefore artificially broadened. While this has a small effect for cosmic shear or galaxy-galaxy lensing studies, which are mostly sensitive to the mean of the distribution, it is potentially problematic for predictions of the angular clustering of galaxies and for the galaxy-bias normalisation. As correction of the broadening, we deconvolved the binned, average posterior for the lens samples as described in Appendix B. For the source samples, where the broadening is not relevant, we directly used the BPZ posteriors.

The two top panels in Fig. 1 show the deconvolved $p(z)$ for all our lens samples. Despite the same photo- $z$ cuts, the differences in the redshift distributions between the lens samples is evident: bluer samples or samples with less stellar mass tend to be more spread out in redshift. The bottom panel plots the average BPZ posteriors (weighted) of all source samples.

\subsection{Average number densities}

We obtain the mean (comoving) number density of lens galaxies with our modified $V_{\max }$-estimator, described in Appendix C. Originally, $V_{\max }$ assumed an exactly determined maximum (comoving) volume $V_{i}$ inside which a galaxy $i$ is observable under the adopted selection criteria; the estimator of the number density was then $\bar{n}_{\mathrm{g}}=\sum_{i=1}^{n} V_{i}^{-1}$ for all $n$ galaxies in the sample (Takeuchi et al. 2000; Schmidt 1968). In our case with photometric redshifts, however, the volume $V_{i}$ is uncertain. Our modified $V_{\max }$ estimator therefore accounts for this uncertainty, and we find values for $\bar{n}_{\mathrm{g}}$ that are then a few per cent larger than the standard $V_{\max }$ based on the best-fit photo- $z$. Our estimates safely ignore a density correction for inhomogeneous galaxy samples because our broad redshift bins at $z>0.1$ in combination with the $\sim 100 \mathrm{deg}^{2}$ survey area span a sufficiently large and homogeneous volume (e.g. Baldry et al. 2012). 
Table 2. Overview of lens samples and selection criteria in addition to star_flag $<1, \mathrm{~T} \_\mathrm{B}>-99$, Flag $<3$, and $i^{\prime} \in[17.5,22.5)$.

\begin{tabular}{lccc}
\hline \hline Sample & $N_{\mathrm{g}}{ }^{(a)}$ & $\bar{z} \pm \sigma_{z}{ }^{(b)}$ & Selection $^{(c)}$ \\
\hline SM1 low- $z$ & $4.96 \times 10^{4}$ & $0.34 \pm 0.11$ & $z_{\mathrm{p}} \in[0.2,0.44], M_{*} \in[0.5,1]$ \\
SM2 low- $z$ & $3.98 \times 10^{4}$ & $0.34 \pm 0.11$ & $z_{\mathrm{p}} \in[0.2,0.44], M_{*} \in[1,2]$ \\
SM3 low- $z$ & $3.01 \times 10^{4}$ & $0.35 \pm 0.10$ & $z_{\mathrm{p}} \in[0.2,0.44], M_{*} \in[2,4]$ \\
SM4 low- $z$ & $2.02 \times 10^{4}$ & $0.36 \pm 0.08$ & $z_{\mathrm{p}} \in[0.2,0.44], M_{*} \in[4,8]$ \\
SM5 low- $z$ & $9.20 \times 10^{3}$ & $0.37 \pm 0.07$ & $z_{\mathrm{p}} \in[0.2,0.44], M_{*} \in[8,16]$ \\
SM6 low- $z$ & $2.46 \times 10^{3}$ & $0.38 \pm 0.07$ & $z_{\mathrm{p}} \in[0.2,0.44], M_{*} \in[16,32]$ \\
RED low- $z$ & $7.15 \times 10^{4}$ & $0.35 \pm 0.08$ & $z_{\mathrm{p}} \in[0.2,0.44], M_{*} \in[0.5,32], u^{*}-r^{\prime}>1.93 z_{\mathrm{p}}+1.85$ \\
BLUE low- $z$ & $7.98 \times 10^{4}$ & $0.34 \pm 0.12$ & $z_{\mathrm{p}} \in[0.2,0.44], M_{*} \in[0.5,32], u^{*}-r^{\prime} \leq 1.93 z_{\mathrm{p}}+1.85$ \\
SM1 high- $z$ & $4.80 \times 10^{4}$ & $0.51 \pm 0.10$ & $z_{\mathrm{p}} \in[0.44,0.6], M_{*} \in[0.5,1]$ \\
SM2 high- $z$ & $5.74 \times 10^{4}$ & $0.51 \pm 0.09$ & $z_{\mathrm{p}} \in[0.44,0.6], M_{*} \in[1,2]$ \\
SM3 high- $z$ & $5.37 \times 10^{4}$ & $0.52 \pm 0.08$ & $z_{\mathrm{p}} \in[0.44,0.6], M_{*} \in[2,4]$ \\
SM4 high- $z$ & $3.78 \times 10^{4}$ & $0.52 \pm 0.08$ & $z_{\mathrm{p}} \in[0.44,0.6], M_{*} \in[4,8]$ \\
SM5 high- $z$ & $1.68 \times 10^{4}$ & $0.52 \pm 0.07$ & $z_{\mathrm{p}} \in[0.44,0.6], M_{*} \in[8,16]$ \\
SM6 high- $z$ & $3.61 \times 10^{3}$ & $0.52 \pm 0.07$ & $z_{\mathrm{p}} \in[0.44,0.6], M_{*} \in[16,32]$ \\
RED high- $z$ & $8.84 \times 10^{4}$ & $0.51 \pm 0.07$ & $z_{\mathrm{p}} \in[0.44,0.6], M_{*} \in[0.5,32], u^{*}-r^{\prime}>1.93 z_{\mathrm{p}}+1.85$ \\
BLUE high- $z$ & $1.29 \times 10^{5}$ & $0.52 \pm 0.09$ & $z_{\mathrm{p}} \in[0.44,0.6], M_{*} \in[0.5,32], u^{*}-r^{\prime} \leq 1.93 z_{\mathrm{p}}+1.85$ \\
\hline
\end{tabular}

Notes. ${ }^{(a)}$ Total number of objects for W1 to W4 combined (effective $92.12 \mathrm{deg}^{2}$ ). ${ }^{(b)}$ Mean and dispersion of maximum-likelihood redshift distribution. ${ }^{(c)}$ Photometric redshift $z_{\mathrm{p}}$, stellar mass $M_{*}$ in units of $h_{70}^{-2} 10^{10} \mathrm{M}_{\odot}$, apparent AB CFHTLenS magnitudes $u^{*}$ and $r^{\prime}$.

Table 3. Overview of source samples and selection criteria in addition to star_flag $<1$, FLAG $<3$, fitsclass $=0$, and $i^{\prime} \in[17.5,24.7)$.

\begin{tabular}{lccccc}
\hline \hline Sample & $N_{\mathrm{g}}{ }^{(a)}$ & $N_{\mathrm{eff}}{ }^{(b)}$ & $\bar{z} \pm \sigma_{z}{ }^{(c)}$ & $2 \mathrm{D} \sigma_{\epsilon}{ }^{(d)}$ & Selection $^{(e)}$ \\
\hline BACK low- $z$ & $2.36 \times 10^{6}$ & $1.94 \times 10^{6}$ & $0.97 \pm 0.28$ & 0.413 & $z_{\mathrm{p}} \in[0.65,1.30]$ \\
BACKa low- $z$ & $1.29 \times 10^{6}$ & $1.08 \times 10^{6}$ & $0.86 \pm 0.26$ & 0.408 & $z_{\mathrm{p}} \in[0.65,0.90]$ \\
BACKb low- $z$ & $1.06 \times 10^{6}$ & $8.63 \times 10^{5}$ & $1.12 \pm 0.22$ & 0.419 & $z_{\mathrm{p}} \in[0.90,1.30]$ \\
BACK high- $z$ & $1.80 \times 10^{6}$ & $1.47 \times 10^{6}$ & $1.03 \pm 0.25$ & 0.413 & $z_{\mathrm{p}} \in[0.75,1.30]$ \\
BACKa high- $z$ & $8.97 \times 10^{5}$ & $7.43 \times 10^{5}$ & $0.93 \pm 0.23$ & 0.407 & $z_{\mathrm{p}} \in[0.75,0.95]$ \\
BACKb high- $z$ & $9.03 \times 10^{5}$ & $7.32 \times 10^{5}$ & $1.14 \pm 0.21$ & 0.420 & $z_{\mathrm{p}} \in[0.95,1.30]$ \\
\hline
\end{tabular}

Notes. ${ }^{(a)}$ Total number of objects for W1 to W4 combined (effective $\left.92.12 \mathrm{deg}^{2}\right)$. ${ }^{(b)}$ Effective number $\left(\sum_{i} w_{i}\right)^{2} / \sum_{i} w_{i}^{2}$ of sources. ${ }^{(c)}$ Mean and dispersion of binned redshift distribution. ${ }^{(d)}$ Weighted ellipticity dispersion $\left\langle|\epsilon|^{2}\right\rangle^{1 / 2}$ of sources. ${ }^{(e)}$ Photometric redshift $z_{\mathrm{p}}$.

Using the $V_{\max }$ densities for CFHTLenS in Fig. 2 (black data points with error bars) as priors improves the convergence of our Monte Carlo analysis because they break parameter degeneracies in the galaxy-bias templates. The priors are log-normal distributions with RMS errors for $\log _{10} \bar{n}_{\mathrm{g}}$ based on jackknife re-sampling (Knight 1999); we removed one MegaCam field at a time for each jackknife sample. The number densities typically range between $2 \times 10^{-4}-4 \times 10^{-3} h^{3} \mathrm{Mpc}^{-3}$ and have a clear trend towards fewer galaxies for higher stellar masses.

Figure 2 also compares our estimates for $\bar{n}_{\mathrm{g}}$ to measured stellar-mass functions (SMFs) from selected studies, which are shown as lines. For this comparison, we convolved the best-fitting SMFs in Wright et al. (2018), Bielby et al. (2012), Davidzon et al. (2013), and Baldry et al. (2012) with our RMS error of $0.27 \mathrm{dex}$ for stellar-mass estimates (log-normal error model), and we then integrated the convolved functions over the stellar-mass ranges in Table 2. The convolution accounts for the Eddington bias, which softens the drop in galaxy counts beyond the characteristic mass in the Schechter function around $5 \times 10^{10} h_{70}^{-2} \mathrm{M}_{\odot}$ and which suppresses the counts below this cutoff. The top axis of the figure indicates, averaged for low- $z$ and high- $z$, the mean stellar masses of our samples. All SMFs in this comparison make assumptions for the stellar population synthesis and the stellar initial mass-function that are consistent with the CFHTLenS stellar masses, and they employ, with the exception of Wright et al. (2018) and partly Davidzon et al. (2013), the same dust extinction law.

That there are still differences between the surveys is probably a sign of inconsistent stellar-mass estimates or galaxy selections. Closest to our selection is the VIPERS study by Davidzon et al. (2013), which identifies galaxies in the patches W1 and W4 of CFHTLS (at that time $\sim 15 \mathrm{deg}^{2}$ ) with our flux limit $i_{\mathrm{AB}}<22.5$. This sample was, superior to our photometric redshift estimates, followed up with the VIMOS multi-object spectrograph at the ESO VLT to obtain precise estimates of stellar mass and redshift with HYPERZMASS (Bolzonella et al. 2010, 2000). In addition to the $i_{\mathrm{AB}}$-band flux limit, they applied a colour cut to exclude low-redshift objects $(z<0.5)$. The resulting SMF in their lowest redshift bin $z \in[0.5,0.6]$ agrees well with our high- $z$ galaxy counts SM5 and SM6, but is $\sim 40 \%$ lower for the less massive galaxies SM1 to SM3. In another study, Bielby et al. (2012) combine deep CFHTLS $\left(\sim 2 \mathrm{deg}^{2}\right)$ photometry with near-infrared observations by the WIRcam Deep Survey (WIRDS) to attain the galaxy SMF at various redshifts, in particular also around our low $-z$ and high- $z$. Again, there is consistency for SM5 and SM6, but our galaxy counts are lower (higher) for 

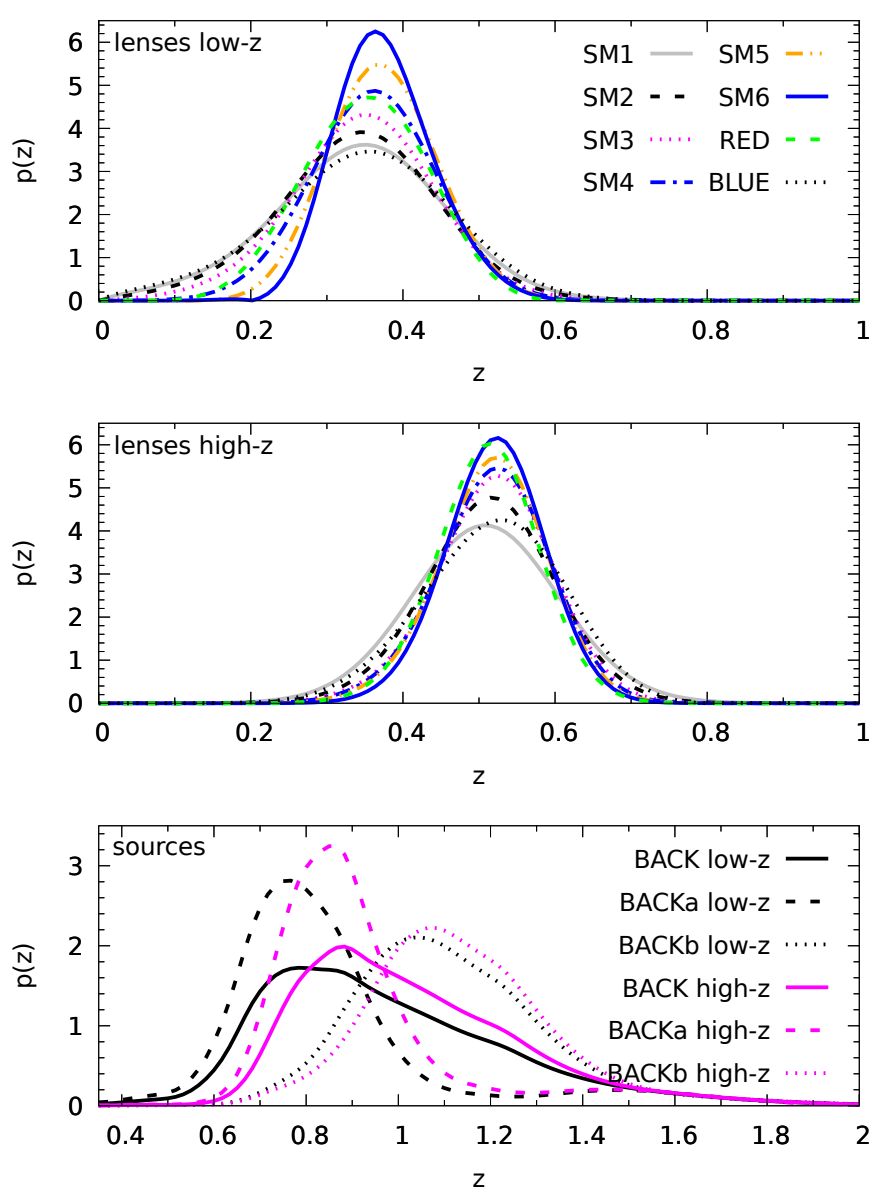

Fig. 1. Redshift distributions $p(z)$ of all galaxy samples. Two top panels: lens samples, low- $z$ and high- $z$, bottom panel: two source samples (solid lines) and their two subsamples (dotted or dashed lines).

low- $z$ (high- $z$ ) in the other stellar-mass bins. This discrepancy for less massive galaxies may be related to their near-infrared colour cuts, which were used to separate stars from galaxies, and the fainter flux-limit of $i=25.5$. Finally, the studies by Wright et al. (2018) and Baldry et al. (2012) are based on the Galaxy And Mass Assembly (GAMA) survey and, in the former case, additionally on the deeper observations in the Cosmos Evolution Survey (COSMOS) and 3D-HST. They consistently find higher galaxy counts for low- $z$, whereas now high- $z$ SM1 to SM4 are in good agreement with our results.

In conclusion, our measurements for $\log _{10} \bar{n}_{\mathrm{g}}$ are broadly supported within $0.2-0.3$ dex by other studies. Mismatches are presumably a mixture of inconsistent selections and stellar mass errors. A clear explanation probably requires a completely consistent selection of galaxies, beyond the scope of this study.

\section{Semi-analytic models of galaxies}

Semi-analytic galaxy models (SAMs) populate matter halos in purely dark matter simulations with galaxies, reducing galaxies and halos to entities with a few global properties that follow a simplified set of physical and empirical laws. By applying prescriptions for a stellar population synthesis and dust extinction the SAM results are, with increased uncertainty, converted into galaxy spectral energy-distributions, and thus mock galaxy catalogues with multi-band photometry. A detailed description of the semi-analytic approach can be found in Lacey et al. (2016),

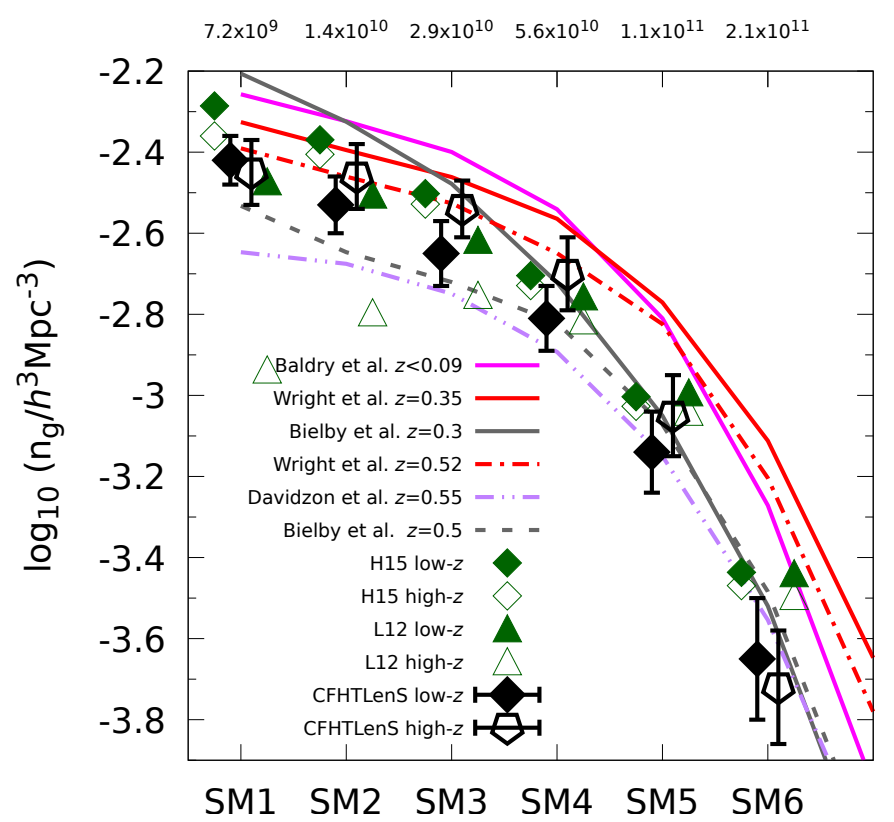

Fig. 2. Comoving number density of galaxies for CFHTLenS (black data points) and the SAMs (green data points) in our stellarmass bins for low- $z$ (filled symbols) and high- $z$ (open symbols). The sample labels SM1 to SM6 are indicated at the bottom; the small numbers at the top are the mean stellar masses inside the bins in units of $h_{70}^{-2} \mathrm{M}_{\odot}$. The coloured lines are the galaxy SMFs by Wright et al. (2018, GAMA/G10-COSMOS/3D-HST), Bielby et al. (2012, WIRDS/CFHTLS), Davidzon et al. (2013, VIPERS/CFHTLS), and Baldry et al. (2012, GAMA). The SMFs are, before integrating over our stellar-mass bins, convolved with a stellar-mass error of $0.27 \mathrm{dex}$ to account for the Eddington bias in our data.

among others. We use the Munich model H15 and the Durham model L12.

\subsection{Simulation and galaxy sample selection}

Our galaxy mocks are based on the Millennium Simulation (Springel et al. 2005). This $N$-body simulation of the dark matter density field has a comoving spatial resolution of $\sim 5 h^{-1} \mathrm{kpc}$ over a cubic volume with $500 h^{-1} \mathrm{Mpc}$ side length, sampled by $\sim 10^{10}$ mass particles. The fiducial cosmology of the simulation $\left(\Omega_{\mathrm{m}}=1-\Omega_{\Lambda}=0.25, \Omega_{\mathrm{b}}=0.045, \sigma_{8}=0.9, h=0.73\right.$, $\left.n_{\mathrm{s}}=1.0\right)$ is different from our fiducial Planck cosmology in Table 4. As shown in Henriques et al. (2017), however, this has only a mild impact on the galaxy clustering because the combination of a higher $\sigma_{8}$ and a lower $\Omega_{\mathrm{m}}$ gives a similar structure growth. We therefore do not expect large differences between the galaxy models and the CFHTLenS galaxy bias from this side.

To compute predictions of the galaxy biasing functions, we combine the galaxy and dark matter power spectra, and their cross-power spectra at the Millennium Simulation snapshots $z=0.3623,0.5086$ for low $-z$ and high- $z$, exactly as described in Sect. 2.3 of SH18. To prepare the various lens samples in these snapshots, we selected SAM galaxies according to Table 2, after adding a random RMS error of 0.27 dex to the SAM stellar masses $\log _{10} M_{*}$ and after converting SDSS magnitudes of the SAMs to CFHTLenS magnitudes according to Sect. 3.2 in Saghiha et al. (2017). By SAM stellar mass we specifically mean that we do not apply BPZ to the SAM photometry to emulate CFHTLenS, rather we utilise the randomised SAM stellar masses. For the colour cut, we identify $z_{\mathrm{p}}$ with the snapshot $z$. 


\subsection{Model differences}

The H15 and L12 models follow somewhat differing SAM approaches and hence produce distinct predictions for the galaxy bias. A major difference is their calibration: H15 fits stellar-mass functions and the red-galaxy fractions as a function of $M_{*}$ to observations for $z \leq 1$; L12 calibrates the model with local luminosity functions (near-infrared and blue bands) and the observed correlation between galactic bulge mass and central black hole mass. In addition, the identification of subhalos in the Millennium Simulation differs and so does the treatment of subhalos and satellite galaxies. L12 satellites, for instance, can merge with a central galaxy while they still have resolved subhalos, whereas H15 satellites have to disrupt their halos first. This directly affects the galaxy clustering on small scales and the radial distribution of satellites inside galaxy clusters (Contreras et al. 2013). Another difference, affecting the photometry of the model galaxies, are the initial mass functions, the implemented models for the stellar population synthesis, and the dust extinction law.

More can be seen in the stellar mass function (SMF) of the models. The green data points in Fig. 2 show the number density of the SAM galaxy samples for the various stellar masses (filled data points: low-z; open data points: high- $z$ ). We obtained these data by dividing the total number of galaxies at the snapshot redshifts by the comoving simulation volume of $\left(500 \mathrm{~h}^{-1} \mathrm{Mpc}\right)^{3}$. Within 0.1 dex these data points closely follow those of CFHTLenS with two exceptions. Firstly, the L12 high$z$ samples (open triangles) have up to 0.5 dex fewer galaxies for SM1 and SM2, due to a strong evolution of the stellar-mass function at low stellar masses. This strong evolution is not present in H15. Secondly, the SAMs predict approximately 0.2 dex more SM6 galaxies for both redshifts.

For the colour-selected samples we find values broadly compatible with CFHTLenS, but also differences between H15 and L12: for RED low- $z$ in H15 (L12), we have $\log _{10}\left(\bar{n}_{\mathrm{g}} / h^{3} \mathrm{Mpc}^{-3}\right)=-2.20(-2.11)$ and for RED high- $z$ $-2.37(-2.37)$. The corresponding figures for BLUE low $z$ and high $-z$ are $-2.02(-2.37)$ and $-1.99(-2.51)$, respectively. L12 thus leans towards a smaller number of blue (SM1 and SM2) galaxies, especially at high-z.

\section{Aperture statistics and reconstruction of biasing functions}

This section briefly summarises the SH18 formalism for galaxybias reconstruction and reports the CFHTLenS aperture statistics to which we apply this technique. The basic idea is to measure the fluctuations of galaxy numbers and lensing convergence inside circular apertures for a range of angular scales. The ratios of their (co-)variances directly probe the projected scaledependent galaxy bias on the sky. By then forward-fitting templates to the observed ratio statistics we reconstruct the average biasing functions $b(k)$ and $r(k)$ for a given fiducial cosmology and galaxy redshift distributions.

\subsection{Fiducial cosmology}

The baseline cosmology in the analysis are the parameters listed in Table 4, taken from the recent cosmic microwave background (CMB) analysis by the Planck Collaboration XIII (2016). In this baseline, we changed the normalisation $\sigma_{8}$ of the matter power spectrum to the smaller value of $\sigma_{8}=0.714$ in order to be consistent with cosmic-shear constraints $\sigma_{8}\left(\Omega_{\mathrm{m}} / 0.27\right)^{0.5} \approx 0.76$ in CFHTLenS (Joudaki et al. 2017; Fu et al. 2014; Heymans et al.
Table 4. Fiducial $\Lambda \mathrm{CDM}$ cosmological model for the galaxy-bias analysis.

\begin{tabular}{ccccccc}
\hline \hline$\Omega_{\mathrm{m}}{ }^{(a)}$ & $\Omega_{\Lambda}{ }^{(b)}$ & $\Omega_{\mathrm{b}}{ }^{(c)}$ & $\sigma_{8}{ }^{(d)}$ & $h^{(e)}$ & $n_{\mathrm{s}}{ }^{(f)}$ & $A_{\mathrm{ia}}{ }^{(g)}$ \\
\hline 0.306 & $1-\Omega_{\mathrm{m}}$ & 0.0479 & 0.714 & 0.683 & 0.965 & -0.48 \\
\hline
\end{tabular}

Notes. The parameters are those from Planck Collaboration XIII (2016) for the TT, TE, and EE CMB power spectrum combined with constraints from baryon acoustic oscillations of galaxies. We changed $\sigma_{8}$ to a lower value, albeit irrelevant for the bias analysis, to be consistent with the cosmic shear constraints in CFHTLenS. ${ }^{(a)}$ Density parameter of dust-like matter. ${ }^{(b)}$ Density parameter of the cosmological constant (chosen for a spatially flat universe). ${ }^{\left({ }^{c}\right)}$ Density parameter of baryonic matter. ${ }^{(d)}$ Normalisation of the linear matter power spectrum. ${ }^{(e)}$ Hubble parameter $H_{0}$ in units of $100 \mathrm{~km} \mathrm{~s}^{-1} \mathrm{Mpc}^{-1} \cdot{ }^{(f)}$ Spectral index of the primordial matter power spectrum. ${ }^{(g)}$ Correlation amplitude of the intrinsic alignment of source galaxies, taken from the CFHTLenS analysis by Heymans et al. (2013).

2013). For the following analysis of the ratio aperture-statistics, however, this smaller value has no practical relevance. This is reflected by the insensitivity of the ratio statistics with respect to any rescaling of the matter power spectrum amplitude.

Nevertheless, the ratio statistics depends weakly on the shape of the matter power spectrum, if it is not a power law in $k$ (van Waerbeke 1998). In our analysis, the power spectrum $P_{\mathrm{m}}(k, \chi)$ at comoving distance $\chi$ is the updated Halofit (Takahashi et al. 2012; Smith et al. 2003), as it is implemented in the latest version of $\mathrm{Nicea}^{3}$ (Kilbinger et al. 2009). In addition, we modify the power spectrum $P_{\mathrm{m}}(k, \chi) \mapsto F(k, \chi) P_{\mathrm{m}}(k, \chi)$ in the non-linear regime by the transfer function $F(k, \chi)$ in Chisari et al. (2018) to correct for AGN feedback, star formation, and adiabatic contraction of matter halos due to gas cooling (Schneider \& Teyssier 2015). Since $F(k, \chi)$ is not well known, we randomly vary $F(k, z)-1$ later on to include systematic errors by baryons.

\subsection{Aperture statistics}

This section is a summary of the relation between power spectra and aperture statistics. For a circular aperture of size $\theta_{\text {ap }}$ at position $\boldsymbol{\theta}$, the density fluctuation of galaxy numbers inside the aperture is

$\mathcal{N}\left(\theta_{\mathrm{ap}} ; \boldsymbol{\theta}\right)=\int \mathrm{d}^{2} \theta^{\prime} U\left(\left|\boldsymbol{\theta}^{\prime}\right| ; \theta_{\mathrm{ap}}\right) \kappa_{\mathrm{g}}\left(\boldsymbol{\theta}^{\prime}+\boldsymbol{\theta}\right)$.

This aperture number count convolves the galaxy numberdensity contrast $\kappa_{\mathrm{g}}(\boldsymbol{\theta})=N(\boldsymbol{\theta}) / \bar{N}_{\mathrm{g}}-1$ of galaxy density $N(\boldsymbol{\theta})$ and mean density $\bar{N}_{\mathrm{g}}$ on the sky with the compensated polynomial filter

$$
\begin{aligned}
& U\left(\theta ; \theta_{\mathrm{ap}}\right)=\frac{1}{\theta_{\mathrm{ap}}^{2}} u\left(\theta \theta_{\mathrm{ap}}^{-1}\right) ; \\
& u(x)=\frac{9}{\pi}\left(1-x^{2}\right)\left(\frac{1}{3}-x^{2}\right) \mathrm{H}(1-x),
\end{aligned}
$$

where $\mathrm{H}(x)$ is the Heaviside step function. The discreteness of galaxies at positions $\boldsymbol{\theta}_{i}$ (i.e. $N(\boldsymbol{\theta})=\sum_{i} \delta_{\mathrm{D}}\left(\boldsymbol{\theta}-\boldsymbol{\theta}_{i}\right)$ for Dirac delta functions $\left.\delta_{\mathrm{D}}(\boldsymbol{\theta})\right)$ gives rise to shot-noise in $\mathcal{N}\left(\theta_{\mathrm{ap}} ; \boldsymbol{\theta}\right)$, which is corrected for in the statistical moments of $\mathcal{N}$ below. The aperture

\footnotetext{
https://github.com/CosmoStat/nicaea
} 
number count $\mathcal{N}$ compares to the aperture mass (i.e. the fluctuation of the lensing convergence $\kappa(\boldsymbol{\theta})$ inside the same aperture):

$M_{\mathrm{ap}}\left(\theta_{\mathrm{ap}} ; \boldsymbol{\theta}\right)=\int \mathrm{d}^{2} \theta^{\prime} U\left(\left|\boldsymbol{\theta}^{\prime}\right| ; \theta_{\mathrm{ap}}\right) \kappa\left(\boldsymbol{\theta}^{\prime}+\boldsymbol{\theta}\right)$.

Our statistical analysis utilises second-order moments of the joint distribution of $\mathcal{N}$ and $M_{\text {ap }}$ over all positions $\boldsymbol{\theta}$. These second-order moments are measures of angular power spectra $P(\ell)$

$$
\begin{aligned}
& \left\langle\mathcal{N}^{2}\right\rangle_{\mathrm{th}}\left(\theta_{\mathrm{ap}} ; b\right)=2 \pi \int_{0}^{\infty} \mathrm{d} \ell \ell P_{\mathrm{n}}(\ell ; b)\left[I\left(\ell \theta_{\mathrm{ap}}\right)\right]^{2}, \\
& \left\langle\mathcal{N} M_{\mathrm{ap}}\right\rangle_{\mathrm{th}}\left(\theta_{\mathrm{ap}} ; b, r\right)=2 \pi \int_{0}^{\infty} \mathrm{d} \ell \ell P_{\mathrm{n} \kappa}(\ell ; b, r)\left[I\left(\ell \theta_{\mathrm{ap}}\right)\right]^{2}, \\
& \left\langle M_{\mathrm{ap}}^{2}\right\rangle_{\mathrm{th}}\left(\theta_{\mathrm{ap}}\right)=2 \pi \int_{0}^{\infty} \mathrm{d} \ell \ell P_{\kappa}(\ell)\left[I\left(\ell \theta_{\mathrm{ap}}\right)\right]^{2},
\end{aligned}
$$

for the angular band-pass filter

$$
I(x)=\frac{12}{\pi} \frac{\mathrm{J}_{4}(x)}{x^{2}} .
$$

We estimate these statistics from observations (see Sect. 4.4), and denote them just by $\left\langle\mathcal{N}^{2}\left(\theta_{\mathrm{ap}}\right)\right\rangle,\left\langle\mathcal{N} M_{\mathrm{ap}}\left(\theta_{\mathrm{ap}}\right)\right\rangle$, and $\left\langle M_{\mathrm{ap}}^{2}\left(\theta_{\mathrm{ap}}\right)\right\rangle$ without the subscript 'th' (for theory) and arguments ' $b$ ' or ' $b, r$ '.

To model the aperture statistics, we use Eqs. (5)-(7) and the following three power-spectra models: $P_{n}(\ell ; b), P_{n k}(\ell ; b, r)$, and $P_{K}(\ell)$. In the models the sources have the probability distribution $p_{\mathrm{s}}(\chi) \mathrm{d} \chi$ in comoving distance $\chi$, and lens galaxies have the distribution $p_{\mathrm{d}}(\chi) \mathrm{d} \chi$. The specific biasing functions of the lens galaxies are given by $b(k)$ and $r(k)$ as indicated by the arguments ' $b$ ' or ' $b, r$ '. There is the angular galaxy power-spectrum

$P_{n}(\ell ; b)=\int_{0}^{\chi_{\mathrm{h}}} \frac{\mathrm{d} \chi p_{\mathrm{d}}^{2}(\chi)}{f_{K}^{2}(\chi)} b^{2}\left(k_{\ell}^{\chi}\right) P_{\mathrm{m}}\left(k_{\ell}^{\chi} ; \chi\right)$,

the galaxy-convergence cross-power

$$
\begin{aligned}
P_{n \kappa}(\ell ; b, r)= & \frac{3 H_{0}^{2} \Omega_{\mathrm{m}}}{2 c^{2}} \\
& \int_{0}^{\chi \mathrm{h}} \frac{\mathrm{d} \chi p_{\mathrm{d}}(\chi) g_{\mathrm{s}}(\chi)}{a(\chi) f_{K}(\chi)} b\left(k_{\ell}^{\chi}\right) r\left(k_{\ell}^{\chi}\right) P_{\mathrm{m}}\left(k_{\ell}^{\chi} ; \chi\right),
\end{aligned}
$$

and the convergence power-spectrum

$P_{\kappa}(\ell)=\frac{9 H_{0}^{4} \Omega_{\mathrm{m}}^{2}}{4 c^{4}} \int_{0}^{\chi_{\mathrm{h}}} \frac{\mathrm{d} \chi g_{\mathrm{s}}^{2}(\chi)}{a^{2}(\chi)} P_{\mathrm{m}}\left(k_{\ell}^{\chi} ; \chi\right)$,

where $f_{K}(\chi)$ is the comoving angular diameter distance,

$g_{\mathrm{s}}(\chi):=\int_{\chi}^{\chi_{\mathrm{h}}} \mathrm{d} \chi^{\prime} p_{\mathrm{s}}\left(\chi^{\prime}\right) \frac{f_{K}\left(\chi^{\prime}-\chi\right)}{f_{K}\left(\chi^{\prime}\right)}$

is a lensing kernel, $k_{\ell}^{\chi}:=(\ell+0.5) / f_{K}(\chi)$ is a wave number at $\chi$, $a(\chi)$ is the scale factor, $\chi_{\mathrm{h}}$ is the maximum distance to a source, and $\mathrm{J}_{n}(x)$ is the $n$ th-order Bessel function of the first kind.

\subsection{Intrinsic alignment and magnification bias}

We add corrections to the theoretical power spectra, Eqs. (5)(7), to account for the intrinsic source alignments (IAs) and the magnification of lenses. To this end, we employ the approximate 'non-linear linear' model for IA (Hirata \& Seljak 2004; Bridle \& King 2007; Joachimi et al. 2011). Specifically, instead of $P_{K}(\ell)$ in the integral Eq. (7), we integrate over

$P_{\kappa}^{\prime}(\ell)=P_{\kappa}(\ell)+P_{\kappa}^{\mathrm{II}}(\ell)+P_{\kappa}^{\mathrm{GI}}(\ell)$,

where the additional intrinsic-intrinsic (II) and shear-intrinsic (GI) terms are ${ }^{4}$

$$
\begin{aligned}
P_{\kappa}^{\mathrm{II}}(\ell) & =\int_{0}^{\chi_{\mathrm{h}}} \frac{\mathrm{d} \chi p_{\mathrm{s}}^{2}(\chi)}{f_{K}^{2}(\chi)} F_{\mathrm{ia}}^{2}(\chi) P_{\mathrm{m}}\left(k_{\ell}^{\chi} ; \chi\right) ; \\
P_{\kappa}^{\mathrm{GI}}(\ell) & =\frac{3 H_{0}^{2} \Omega_{\mathrm{m}}}{c^{2}} \int_{0}^{\chi \mathrm{h}} \frac{\mathrm{d} \chi p_{\mathrm{s}}(\chi) g_{\mathrm{s}}(\chi)}{a(\chi) f_{K}(\chi)} F_{\mathrm{ia}}(\chi) P_{\mathrm{m}}\left(k_{\ell}^{\chi} ; \chi\right),
\end{aligned}
$$

and

$$
F_{\mathrm{ia}}(\chi) \approx-2.4 \times 10^{-2}\left(\frac{A_{\mathrm{ia}}}{3.0}\right)\left(\frac{\Omega_{\mathrm{m}}}{0.3}\right)\left(\frac{D_{+}(\chi)}{0.5}\right)^{-1}
$$

uses the linear growth factor of structure $D_{+}(\chi)$, normalised by $D_{+}(\chi=0)$ (Peebles 1980). The parameter $A_{\text {ia }}$ defines the amplitude of the intrinsic alignments in the model. In our Bayesian analysis we use as prior $A_{\mathrm{ia}}=-0.48_{-0.87}^{+0.75}$ the constraints from Heymans et al. (2013) (68\% credible interval, hereafter CI).

Likewise, we correct for the magnification of the lens number density by foreground structure in the power spectrum $P_{n \kappa}(\ell ; b, r)$, Eq. (6), through

$P_{n \kappa}^{\prime}(\ell ; b, r)=P_{n \kappa}(\ell ; b, r)+P_{n \kappa}^{\mathrm{GI}}(\ell ; b, r)+P_{n \kappa}^{\mathrm{mb}}(\ell)$

with

$$
P_{n \kappa}^{\mathrm{GI}}(\ell ; b, r)=\int_{0}^{\chi_{\mathrm{h}}} \frac{\mathrm{d} \chi p_{\mathrm{s}}(\chi) p_{\mathrm{d}}(\chi)}{f_{K}^{2}(\chi)} b\left(k_{\ell}^{\chi}\right) r\left(k_{\ell}^{\chi}\right) F_{\mathrm{ia}}(\chi) P_{\mathrm{m}}\left(k_{\ell}^{\chi} ; \chi\right)
$$

and

$$
P_{n \kappa}^{\mathrm{mb}}(\ell)=-\frac{9 H_{0}^{4} \Omega_{\mathrm{m}}^{2}}{2 c^{4}} \int_{0}^{\chi_{\mathrm{h}}} \mathrm{d} \chi \frac{g_{\mathrm{s}}(\chi) g_{\mathrm{d}}(\chi)}{a^{2}(\chi)} P_{\mathrm{m}}\left(k_{\ell}^{\chi} ; \chi\right),
$$

where

$g_{\mathrm{d}}(\chi):=\int_{\chi}^{\chi_{\mathrm{h}}} \mathrm{d} \chi^{\prime} p_{\mathrm{d}}\left(\chi^{\prime}\right) \frac{f_{K}\left(\chi^{\prime}-\chi\right)}{f_{K}\left(\chi^{\prime}\right)}$

is the lensing kernel of the foreground structure (Unruh et al. 2020; Hilbert et al. 2009; Hartlap 2009). The expression $P_{n \kappa}^{\mathrm{mb}}(\ell)$ assumes a volume-complete sample of lens galaxies which is approximately correct for our stellar-mass limited samples. The flux limit of $i^{\prime}<22.5$ mostly affects the samples SM1 and SM2 for low $z$ and high- $z$ (Sect. 3.2 in Simon et al. 2013). The estimated magnification bias for $\left\langle\mathcal{N} M_{\text {ap }}\right\rangle$ can reach levels of several per cent for lenses with low clustering amplitude and high redshifts $(z \sim 0.5)$ and should therefore be modelled. The impact of magnification on $\left\langle\mathcal{N}^{2}\right\rangle$, on other hand, is negligible but nevertheless included here (using Eq. (36) in SH18).

4 In Simon \& Hilbert (2018) there is a missing minus sign for $F_{\text {ia }}(\chi)$ in their Eq. (31) and a typo in the pre-factor of $P_{n k}^{\mathrm{mb}}(\ell) \equiv P_{n k}^{(2)}(\ell)$ in their Eq. (34), which have both been corrected here. The pre-factor of the first integral in their Eq. (36) should be $9 H_{0}^{4} c^{-4} \Omega_{\mathrm{m}}^{2}$. 


\subsection{Estimators and data covariance}

The second-order moments of the aperture statistics are linear transformations of the angular correlations functions for the lens clustering $\omega(\theta)$, mean tangential shear around lenses $\bar{\gamma}_{\mathrm{t}}(\theta)$, and the cosmic shear $\xi_{ \pm}(\theta)$, which all can be obtained relatively straightforwardly from observational data (e.g. Simon et al. 2007). To perform this transformation we measure with Athena ${ }^{5}$ the correlation functions for angular separations between 9 arcsec and $3 \mathrm{deg}$ in 8000 bins in the four patches W1 to W4 and combine the measurements (Kilbinger et al. 2014). The result is statistics for $n=8$ aperture radii $\theta_{\text {ap }}$ between 2 and 90 arcmin, equally spaced on a logarithmic scale.

The largest aperture size is our choice to retain a reasonably large number of jackknife samples; the resampling scheme removes subfields that stretch the full angular range of the correlation functions. The aim of the smallest aperture size, on the other hand, is to keep the bias in the estimated statistics below $10 \%$ at a few arcmin. The bias arises due to the blending of galaxy images which compromises lensfit below 9 arcsec, our smallest galaxy separation, and hence produces a gap in the correlation functions (Miller et al. 2013; Heymans et al. 2012). Previous studies set the correlation signal to zero inside the gap and produced a 'transformation bias' in the aperture statistics. We follow a revised, even though not perfect, approach with less transformation bias.

Specifically, we extrapolate the correlations below 9 arcsec in two different ways, depending on which correlation function is used. For $\bar{\gamma}_{\mathrm{t}}(\theta)$ and $\xi_{ \pm}(\theta)$, which are respectively converted into $\left\langle\mathcal{N} M_{\mathrm{ap}}\right\rangle$ and $\left\langle M_{\mathrm{ap}}^{2}\right\rangle$, we extend the lowest angular bin to 1 arcsec, which means that we assume a constant signal equal to that at 9 arcsec. For $\omega(\theta)$, which is converted into $\left\langle\mathcal{N}^{2}\right\rangle$, we fit a power law $\omega(\theta) \propto \theta^{-\delta}$ to the data points between $9 \operatorname{arcsec} \leq \theta \leq 1 \mathrm{arcmin}$, and we then extrapolate the best fit to $0.1 \operatorname{arcsec} \leq \theta \leq 9 \operatorname{arcsec}$ in the transformation. A power-law correlation function for galaxy clustering is a sensible approximation on small scales (e.g. Zehavi et al. 2005). To quantify the remaining transformation bias in the estimated aperture statistics after application of these extrapolations, we use mock galaxy (SM1-SM6, RED, and BLUE low- $z$ and high- $z$ ) and shear catalogues that were constructed from the $\mathrm{H} 15$ data and previously used for the work in SH18. With these mocks we compare the estimated aperture statistics (with extrapolation) to the true aperture statistics (with no truncation at 9 arcsec). In summary, the transformation bias for the aperture moments $\left\langle\mathcal{N} M_{\mathrm{ap}}\right\rangle$ and $\left\langle M_{\mathrm{ap}}^{2}\right\rangle$ is typically below $1 \%$ at $\theta_{\text {ap }} \approx 2 \operatorname{arcmin}$. For $\left\langle\mathcal{N}^{2}\right\rangle$ it is about $10 \%$ at $\theta_{\text {ap }} \approx 2 \operatorname{arcmin}$ (biased low), but quickly falls below a few per cent beyond several arcmin.

For estimates of the errors in the aperture statistics, we perform jackknife resampling of the data (Singh et al. 2017; Norberg et al. 2009; Knight 1999). The resulting jackknife covariance matrix,

$\mathrm{C}=\frac{n_{\mathrm{jk}}-1}{n_{\mathrm{jk}}} \sum_{i=1}^{n_{\mathrm{jk}}}\left(\boldsymbol{d}_{i}-\overline{\boldsymbol{d}}\right)\left(\boldsymbol{d}_{i}-\overline{\boldsymbol{d}}\right)^{t} ; \overline{\boldsymbol{d}}=\frac{1}{n_{\mathrm{jk}}} \sum_{i=1}^{n_{\mathrm{jk}}} \boldsymbol{d}_{i}$,

for $i=1 \ldots n_{\mathrm{jk}}$ jackknife samples,

$d_{i}=\left(d_{i}^{(1)}, d_{i}^{(2)}, d_{i}^{(3)}\right)$,

comprises the error covariances between different angular scales and types of the aperture statistics, namely $d_{i j}^{(1)}=\left\langle\mathcal{N}^{2}\right\rangle_{i}\left(\theta_{\mathrm{ap}, j}\right)$,

\footnotetext{
5 http://www. cosmostat. org/software/athena
}

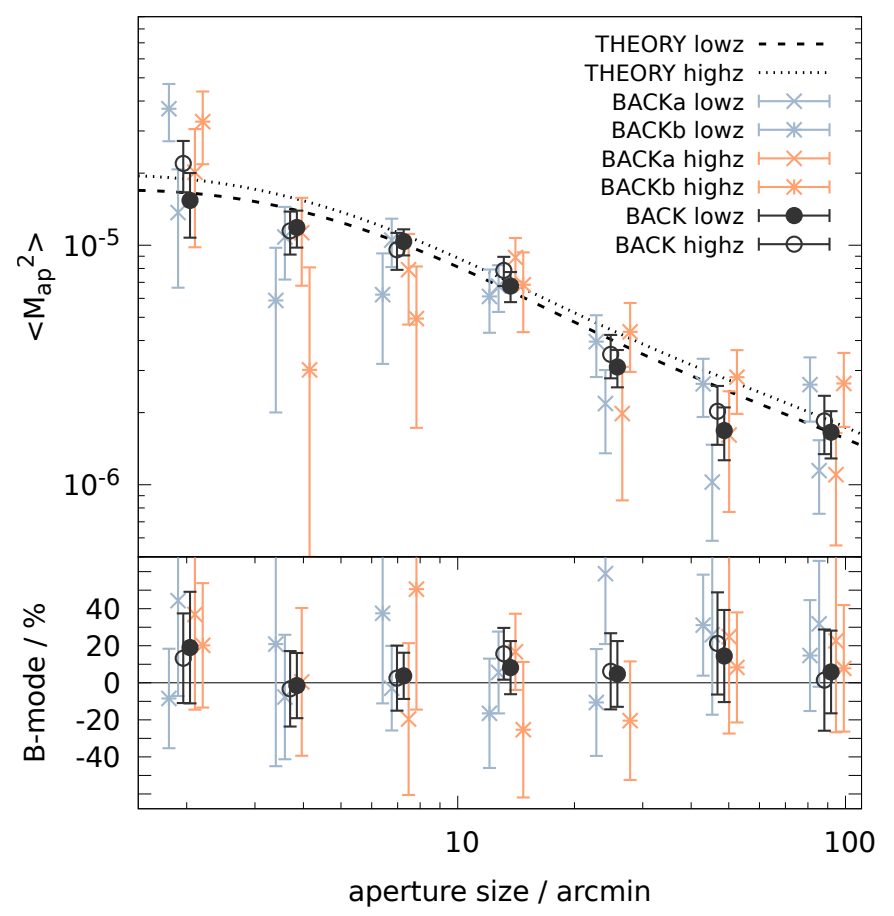

Fig. 3. Variance $\left\langle M_{\mathrm{ap}}^{2}\right\rangle$ as function of aperture size for our source samples. Top panel: E-mode and the predictions (lines) for the BACK low- $z$ and BACK high- $z$ data points (solid and open circles). The crosses and stars correspond to the (noisier) signals of the four subdivisions $a$ and $b$ of the source samples. Bottom panel: B-mode relative to the E-mode signal (in percentage). This plot uses seven evenly spaced aperture radii instead of the eight used in the analysis.

$d_{i j}^{(2)}=\left\langle\mathcal{N} M_{\mathrm{ap}}\right\rangle_{i}\left(\theta_{\mathrm{ap}, j}\right)$, and $d_{i j}^{(3)}=\left\langle M_{\mathrm{ap}}^{2}\right\rangle_{i}\left(\theta_{\mathrm{ap}, j}\right)$. For each jackknife sample, we remove two adjacent MegaCam pointings from one field, redo the measurement, and finally combine the results from all four patches W1 to W4 into $\boldsymbol{d}_{i}$. In three samples, where the fields have an odd number of pointings, we remove three pointings. In total, we thereby obtain $n_{\mathrm{jk}}=63$ jackknife samples to estimate the error covariance.

Figure 3 shows our measurements for $\left\langle M_{\mathrm{ap}}^{2}\right\rangle$ and the jackknife error; the top panel is the E-mode signal, the bottom panel the B-mode signal, which is consistent with zero, relative to the E-mode. In our analysis the measurements for BACK low$z$ and BACK high- $z$ are plotted as circles. The noisier data of the source-sample subdivisions $a$ and $b$ are depicted as crosses and stars. Importantly, the circle data points are the weighted average of crosses, stars, and the cross-correlation signal of the subdivisions (not shown here). The dashed and dotted lines are predictions based the cosmology in Table 4.

\subsection{Ratio statistics and projected galaxy bias}

The ratio statistics

$$
\begin{aligned}
& \hat{b}_{2 \mathrm{D}}\left(\theta_{\mathrm{ap}}\right)=\sqrt{\frac{\left\langle\mathcal{N}^{2}\right\rangle\left(\theta_{\mathrm{ap}}\right)}{\left\langle M_{\mathrm{ap}}^{2}\right\rangle\left(\theta_{\mathrm{ap}}\right)}} ; \\
& \hat{r}_{2 \mathrm{D}}\left(\theta_{\mathrm{ap}}\right)=\frac{\left\langle\mathcal{N} M_{\mathrm{ap}}\right\rangle\left(\theta_{\mathrm{ap}}\right)}{\sqrt{\left\langle\mathcal{N}^{2}\right\rangle\left(\theta_{\mathrm{ap}}\right)\left\langle M_{\mathrm{ap}}^{2}\right\rangle\left(\theta_{\mathrm{ap}}\right)}}
\end{aligned}
$$

is the observed projection of the biasing functions $b(k)$ and $r(k)$ on the sky. These raw data are plotted in Fig. 4, as function of aperture size, for CFHTLenS with the bias factor $\hat{b}_{2 \mathrm{D}}$ in 

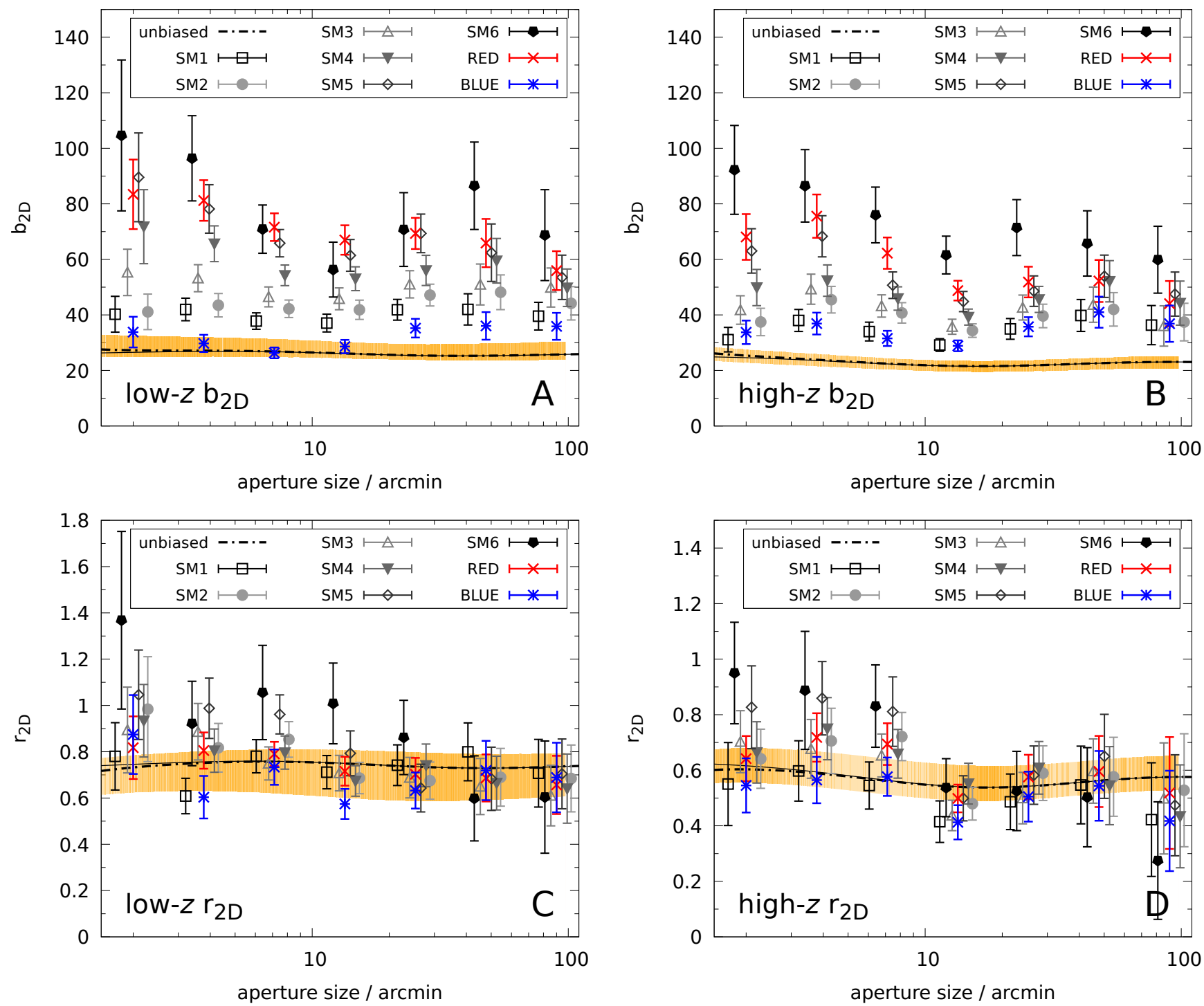

Fig. 4. Observed ratio statistics with jackknife errors for the CFHTLenS lens samples in the low- $z$ bin (panels $A$ and $C$ ) and the high- $z$ bin (panels $B$ and $D$ ). The source samples are BACK low- $z$ and BACK high- $z$, respectively. The data points are slightly shifted along the $x$-axis for better visibility. The dashed lines are predictions for unbiased galaxies (the galaxy-bias normalisation) with SM3 $p_{\mathrm{d}}(z)$. The shaded regions bracket the prediction variations using the $p_{\mathrm{d}}(z)$ of the other lens samples. Top panels: relative clustering $\hat{b}_{2 \mathrm{D}}\left(\theta_{\mathrm{ap}}\right)$ of galaxies and matter. Bottom panels: correlation $\hat{r}_{2 \mathrm{D}}\left(\theta_{\mathrm{ap}}\right)$ between the (projected) galaxy and matter distribution. This plot uses seven evenly spaced aperture sizes instead of the eight used in the analysis.

the top panels $\mathrm{A}$ and $\mathrm{B}$, and the correlation coefficient $\hat{r}_{2 \mathrm{D}}$ in the bottom panels $\mathrm{C}$ and $\mathrm{D}$. The error bars indicate symmetric jackknife errors, whereas the analysis below accounts for asymmetric errors in the ratio statistics through a more complex likelihood model. The likelihood model also accounts for the correlation between the data points at different aperture sizes, encoded in the jackknife covariance. Moreover, statistical errors for different lens samples are correlated because they share the same shear data.

The lines and shaded areas inside the panels are predictions for unbiased galaxies and the fiducial cosmology in Table 4. They are traditionally used as normalisation for the projected galaxy bias to correct, among other things, for the distinct radial projection kernels of galaxies and matter (Hoekstra et al. 2002). Specifically, the dashed lines are the normalisation for SM3 low$z$ (left panels) and high- $z$ (right panels). The normalisation varies weakly with aperture size, and therefore essentially rescales the amplitude of the inferred galaxy bias. This is also the case for the other samples. Across all lens samples in the same photo$z$ bin, however, the normalisation amplitude varies by about $10 \%$ within the shaded regions owing to differences among the $p_{\mathrm{d}}(z)$ (Fig. 1). The predictable uncertainties in the normalisation amplitude for a specific galaxy sample are estimators for the systematic error in the reconstructed biasing functions. This is exploited below.

Coming back to the CFHTLenS data points, our samples are usually more strongly clustered than unbiased galaxies (i.e. $\hat{b}_{2 \mathrm{D}}$ is larger). In addition, our galaxy samples are strongly correlated with the matter-density field, which means that $\hat{r}_{2 \mathrm{D}}$ is consistent with unbiased galaxies. Inside large apertures the correlation is similar for all lens samples, whereas there is some up-scatter below 20 arcmin. This hints at sample differences in the galaxy biasing at small scales. To investigate this in detail we deproject the aperture statistics. 
Table 5. Template parameters.

\begin{tabular}{lc}
\hline \hline Param & Description \\
\hline$b_{\mathrm{ls}}$ & Large-scale bias factor \\
$r_{\mathrm{ls}}$ & Large-scale correlation factor \\
$b(m)$ & Mean biasing function (interp.) \\
$V(m)$ & Normalised excess-variance (interp.) \\
$m_{\text {piv }}$ & $\left\langle N \mid m_{\text {piv }}\right\rangle=1 ;$ pivotal halo mass \\
$f_{\text {cen }}$ & Halo fraction open for central galaxies \\
$\zeta$ & Concentration parameter of satellites \\
\hline
\end{tabular}

Notes. The HOD functions $b(m)$, related to $\langle N \mid m\rangle$, and $V(m)$, related to $\langle N(N-1) \mid m\rangle$, cover the mass range $10^{4}-10^{16} h^{-1} \mathrm{M}_{\odot}$ and are interpolated with 22 points each.

\subsection{Template models for deprojection}

To reconstruct spatial biasing functions from the projected ratio statistics in Fig. 4 we fit the models

$$
\begin{aligned}
& \hat{b}_{2 \mathrm{D}}\left(\theta_{\mathrm{ap}} ; b\right)=\sqrt{\frac{\left\langle\mathcal{N}^{2}\right\rangle_{\mathrm{th}}\left(\theta_{\mathrm{ap}} ; b\right)}{\left\langle M_{\mathrm{ap}}^{2}\right\rangle_{\mathrm{th}}\left(\theta_{\mathrm{ap}}\right)}} ; \\
& \hat{r}_{2 \mathrm{D}}\left(\theta_{\mathrm{ap}} ; b, r\right)=\frac{\left\langle\mathcal{N} M_{\mathrm{ap}}\right\rangle_{\mathrm{th}}\left(\theta_{\mathrm{ap}} ; b, r\right)}{\sqrt{\left\langle\mathcal{N}^{2}\right\rangle_{\mathrm{th}}\left(\theta_{\mathrm{ap}} ; b\right)\left\langle M_{\mathrm{ap}}^{2}\right\rangle_{\mathrm{th}}\left(\theta_{\mathrm{ap}}\right)}}
\end{aligned}
$$

to the measurements by using templates for $b(k)$ and $r(k)$. Templates are useful here because, due to the smoothing in the projections (24), a deprojection into $b(k)$ and $r(k)$ for a given $\hat{b}_{2 \mathrm{D}}$ and $\hat{r}_{2 \mathrm{D}}$ without regularisation is unstable. We denote the templates $b(k ; \boldsymbol{\Theta})$ and $r(k ; \boldsymbol{\Theta})$ to indicate the set $\boldsymbol{\Theta}$ of parameters they are based on.

We employ templates that are thoroughly tested with mock galaxy survey data, similar to our CFHTLenS data, in SH18. They are based on the halo model in Seljak (2000) but with more freedom for the halo-occupation distribution (HOD) of galaxies and a model-free galaxy bias on linear scales. This halo model ignores relevant physics, such as halo exclusion or assembly bias or galactic subhalos, and it has a crude treatment of central galaxies. Nevertheless, the templates produce an accurate deprojection of the biasing functions which is our primary interest here. We outline the template construction in Appendix D and restrict ourselves here to a brief description of the template parameters, listed in Table 5.

The templates have 49 free parameters $\boldsymbol{\Theta}=$ $\left(\ldots, b_{i}, \ldots, V_{i}, \ldots, \zeta, m_{\mathrm{piv}}, b_{\mathrm{ls}}, r_{\mathrm{ls}}, f_{\mathrm{cen}}\right)$. The large number of parameters is due to the fine sampling of the HOD mean number of galaxies, $\langle N \mid m\rangle$, and galaxy pairs, $\langle N(N-1) \mid m\rangle$, with 22 interpolation points each that cover the broad halo mass range $10^{4} \leq m \leq 10^{16} h^{-1} \mathrm{M}_{\odot}$. Our intention is to keep the HOD as flexible as possible and to avoid a model bias in the deprojection with a too restrictive HOD. The meaning of the parameters is as follows:

- The two parameters $b_{\mathrm{ls}}=b(k \rightarrow 0)$ and $r_{\mathrm{ls}}=r(k \rightarrow 0)$ quantify the linear stochastic bias on large (linear) scales. They are completely independent from the galaxy bias on small scales, i.e. independent from the template HOD;

- The parameter $f_{\text {cen }} \in[0,1]$ is the fraction of halos that permit a central galaxy in the selected galaxy population. This fraction is not to be confused with the fraction of central galaxies in a sample;

- A concentration parameter $\zeta \neq 1$ indicates biased satellite galaxies that have, averaged over all halos, a different distri- bution from the dark matter inside halos. For $\zeta>1$ satellites have a more diffuse distribution, and for $\zeta<1$ a more concentrated distribution;

- The pivotal mass $m_{\text {piv }}$ defines the halo mass scale, where $\left\langle N \mid m_{\text {piv }}\right\rangle=1$ and $N$ comprises both central and satellite galaxies;

- The mean number of galaxies in a halo of mass $m,\langle N \mid m\rangle=$ $b(m) m /\left(b\left(m_{\text {piv }}\right) m_{\text {piv }}\right)$, is expressed by the mean biasing function $b(m)$ and its value at $m_{\text {piv }}$. The $b(m)$ is linearly interpolated on a logarithmic $m$-scale with 22 interpolation points, and it has to satisfy the normalisation condition in Eq. (D.12).

- The mean number of galaxy pairs, expressed by the normalised excess variance $V(m)$ via $\langle N(N-1) \mid m\rangle=$ $\langle N \mid m\rangle[\langle N \mid m\rangle+V(m)]$, is sensitive to the variance of galaxy numbers inside halos, which means for $V(m)=0$ that galaxy numbers have a Poisson variance, for $V(m)>0$ a superPoisson variance, and for $V(m)<0$ a sub-Poisson variance. Similar to $b(m), V(m)$ is interpolated with 22 points. To be physically meaningful, the function $V(m)$ has to be larger than $\max \{-1,-\langle N \mid m\rangle\}$, which is accounted for in the template fitting.

Unbiased galaxies have $b_{\mathrm{ls}}=r_{\mathrm{ls}}=\zeta=1, f_{\text {cen }}=0, b(m)=1$, and $V(m)=0$ for all halo masses $m$.

The template parameter space is highly degenerate, which may pose numerical problems when sampling the space during our deprojection technique. Therefore, we also fit the relation

$\bar{n}_{\mathrm{g}}(\boldsymbol{\Theta})=\int_{0}^{\infty} \mathrm{d} m n(m)\langle N \mid m\rangle=\frac{\Omega_{\mathrm{m}} \bar{\rho}_{\text {crit }}}{m_{\text {piv }} b\left(m_{\text {piv }}\right)}$

to our observed $\bar{n}_{\mathrm{g}}$ in Fig. 2 to better confine $m_{\text {piv }}$ and to break parameter degeneracies ${ }^{6}$. Here, $\bar{\rho}_{\text {crit }}=3 H_{0}^{2} /\left(8 \pi G_{\mathrm{N}}\right)$ denotes the critical matter density at $z=0$. Equation (25) follows from the definition of $b(m)$ and the condition Eq. (D.12).

\subsection{Bayesian reconstruction of biasing functions}

We forward-fit in a Bayesian analysis the templates $b(k ; \boldsymbol{\Theta})$ and $r(k ; \boldsymbol{\Theta})$ to the ratio statistics (Eq. (23)); the vector $\boldsymbol{\Theta}$ comprises all template parameters in Table 5. For the likelihood function of the data we assume a Gaussian multivariate error distribution of the data,

$\boldsymbol{d}=\left(\ldots,\langle\mathcal{N}\rangle\left(\theta_{\mathrm{ap}, i}\right), \ldots,\left\langle\mathcal{N} M_{\mathrm{ap}}\right\rangle\left(\theta_{\mathrm{ap}, i}\right), \ldots,\left\langle M_{\mathrm{ap}}^{2}\right\rangle\left(\theta_{\mathrm{ap}, i}\right), \ldots\right)$,

and a lognormal error distribution of the galaxy number density $\bar{n}_{\mathrm{g}}$. Specifically, the probability distribution function $p_{\delta}$ of statistical errors $\delta \boldsymbol{d}$ and $\delta_{n}$ in $\boldsymbol{d}$ and $\log _{10} \bar{n}_{\mathrm{g}}$, respectively, is

$$
-2 \ln p_{\delta}\left(\delta \boldsymbol{d}, \delta_{n}\right)=\mathrm{const}+n_{\mathrm{jk}} \ln \left(1+\frac{\delta \boldsymbol{d}^{\mathrm{T}} \mathrm{C}^{-1} \delta \boldsymbol{d}}{n_{\mathrm{jk}}-1}\right)+\frac{\delta_{n}^{2}}{\sigma_{\operatorname{logn}}^{2}},
$$

for the error covariance $C$ of $\boldsymbol{d}$ (Sect. 4.4) and an error variance $\sigma_{\log n}$ of $\log _{10} \bar{n}_{\mathrm{g}}$ (error bars in Fig. 2). This noise model also marginalises over statistical errors in our estimate of $C$, which is constructed from $n_{\mathrm{jk}}$ jackknife samples (Sellentin \& Heavens 2016). As discussed in SH18, the posterior density of the template parameters given the ratio statistics in Eq. (23) is then a

\footnotetext{
6 An alternative route without the extra parameter $m_{\text {piv }}$ would be to use the relation $\langle N \mid m\rangle=m b(m) \bar{n}_{\mathrm{g}} \bar{\rho}_{\mathrm{m}}^{-1}, \bar{\rho}_{\mathrm{m}}=\Omega_{\mathrm{m}} \bar{\rho}_{\text {crit }}$, and the measured $\bar{n}_{\mathrm{g}}$ with marginalisation over the statistical error in $\bar{n}_{\mathrm{g}}$.
} 
marginalisation of $p_{\delta}$ with respect to $\boldsymbol{x}:=\left(\ldots,\left\langle M_{\mathrm{ap}}^{2}\right\rangle\left(\theta_{\mathrm{ap}, i}\right), \ldots\right)$,

$$
\begin{array}{r}
p\left(\boldsymbol{\Theta} \mid \boldsymbol{d}, \bar{n}_{\mathrm{g}}\right) \propto p_{\Theta}(\boldsymbol{\Theta}) \int \mathrm{d} \boldsymbol{x} p_{\delta}(\boldsymbol{d}-\boldsymbol{m}(\boldsymbol{\Theta}, \boldsymbol{x}), \\
\left.\log _{10} \frac{\bar{n}_{\mathrm{g}}}{\bar{n}_{\mathrm{g}}(\boldsymbol{\Theta})}\right) p_{x}(\boldsymbol{x}),
\end{array}
$$

where $\boldsymbol{m}(\boldsymbol{\Theta}, \boldsymbol{x})=\left(\boldsymbol{m}^{(1)}, \boldsymbol{m}^{(2)}, \boldsymbol{m}^{(3)}\right)$ and

$m_{i}^{(1)}=\hat{b}_{2 \mathrm{D}}^{2}\left(\theta_{\mathrm{ap}, i} ; b\right) x_{i} ;$

$m_{i}^{(2)}=\hat{b}_{2 \mathrm{D}}\left(\theta_{\mathrm{ap}, i} ; b\right) \hat{r}_{2 \mathrm{D}}\left(\theta_{\mathrm{ap}, i} ; b, r\right) x_{i}$;

$m_{i}^{(3)}=x_{i}$.

The integral kernel $p_{\delta}(\ldots)$ inside (28) is the likelihood of $\boldsymbol{d}$ given $\boldsymbol{\Theta}$, while $p_{\Theta}(\boldsymbol{\Theta})$ and $p_{x}(\boldsymbol{x})$ are prior densities for $\boldsymbol{\Theta}$ and $x_{i}$. The prior densities are detailed in the following section.

Since we seek constraints on the biasing functions rather than $\boldsymbol{\Theta}$, we convert the posterior density of $\boldsymbol{\Theta}$ into a posterior distribution for $b(k)$ and $r(k)$ by drawing $\boldsymbol{\Theta}_{i} \sim p\left(\boldsymbol{\Theta} \mid \boldsymbol{d}, \bar{n}_{\mathrm{g}}\right)$ from (28) in a Monte Carlo process and by computing the mean and variance of $\left\{b\left(k ; \boldsymbol{\Theta}_{i}\right)\right\}$ and $\left\{r\left(k ; \boldsymbol{\Theta}_{i}\right)\right\}$ for a range of $k$.

\subsection{Bayesian priors}

For the prior densities $p_{\Theta}(\Theta)=\prod_{i} p_{\Theta_{i}}\left(\Theta_{i}\right)$ and $p_{x}(\boldsymbol{x})=$ $\prod_{i} p_{x_{i}}\left(x_{i}\right)$ in Eq. (28), we assert, similar to the analysis in SH18, uniform top-hat priors and the boundaries $V(m) \in$ $[\max \{-1,-\langle N \mid m\rangle\}, 1], \zeta \in[0.1,2], \log _{10}\left(m_{\text {piv }} h \mathrm{M}_{\odot}^{-1}\right) \in[4,16]$, $b_{\mathrm{ls}} \in[0,5], r_{\mathrm{ls}} \in[0,2]$, and $f_{\text {cen }} \in[0,1]$.

An exception here is the prior density for $b_{i}:=b\left(m_{i}\right)$, which compared to SH18 now has the less informative density $p_{b_{i}}\left(b_{i}\right) \propto$ $1 / b_{i}$ or, equivalently, $p_{b_{i}}\left(\ln b_{i}\right)=$ const. This change is needed to avoid a bias in the $r(k)$ reconstruction for $k \gtrsim 3 h^{-1} \mathrm{Mpc}^{-1}$ due to the noisier CFHTLenS data (for more details on this problem, see Appendix E). Likewise, the prior density for $x_{i}$ is now $p_{x}(\boldsymbol{x}) \propto 1 / \prod_{i} x_{i}$.

\subsection{Systematic error of the galaxy-bias amplitude}

Errors in the fiducial cosmology or the redshift distributions of lenses and sources (i.e. in the projection kernel) cause systematic errors in the amplitude of the reconstructed biasing functions $b(k)$ and $r(k)$. Since changes in the projection kernel produce variations in the amplitudes of $\hat{b}_{2 \mathrm{D}}$ and $\hat{r}_{2 \mathrm{D}}$ with only weak dependence on angular scale, the $k$-dependence of errors in the deprojected biasing functions is also weak, and the amplitude errors in the deprojection are of similar magnitude. We therefore estimate errors in $b(k)$ and $r(k)$ by propagating variations in the lensing kernel parameters to $\hat{b}_{2 \mathrm{D}}$ and $\hat{r}_{2 \mathrm{D}}$ for unbiased galaxies (i.e. the normalisation for the projected bias), and we average their RMS variations over $1^{\prime} \lesssim \theta_{\text {ap }} \lesssim 2^{\circ}$.

As baseline for the error model we assume the RMS values in Table 6 for (i) the mean and width of the redshift distributions, (ii) the set of fiducial cosmological parameters, (iii) the baryon physics in the non-linear matter power spectrum, and (iv) the amplitude $A_{\text {ia }}=-0.48_{-0.87}^{+0.75}$ of the IA, based on the CFHTLenS constraints in Heymans et al. (2013). The error distribution of cosmological parameters for (ii) is the posterior distribution of the Planck TT, TE, and EE cosmology constraints combined with data from BAO experiments; the reference cosmology is a flat $w$ CDM model (Planck Collaboration XIII
Table 6. Baseline of assumed systematic errors for the reconstruction of biasing functions.

\begin{tabular}{lc}
\hline \hline Source & RMS error \\
\hline Redshift bias (sources and lenses) & $2.0 \%$ \\
Width of $p(z)$ (sources and lenses) & $5.0 \%$ \\
$\Omega_{\mathrm{m}}$ & $4.3 \%$ \\
$\Omega_{\mathrm{b}}$ & $4.8 \%$ \\
$w$ & $6.2 \%$ \\
$H_{0}$ & $2.3 \%$ \\
$n_{\mathrm{s}}$ & $0.5 \%$ \\
$\sigma_{8}$ & $2.6 \%$ \\
$A_{\mathrm{ia}}$ & $170 \%$ \\
$F(k, z)$ & $30.0 \%$ \\
\hline
\end{tabular}

Notes. The resulting systematic RMS uncertainties of $b(k)$ and $r(k)$ amplitudes are within $[6.8 \%, 13.5 \%]$ and $[5.2 \%, 8.5 \%]$, respectively (see text). Correlated errors on cosmological parameters $\Omega_{\mathrm{m}}$ to $\sigma_{8}$ are the posterior constraints from Planck Collaboration XIII (2016) for a flat $w \mathrm{CDM}$ model. The IA parameter is $A_{\mathrm{ia}}=-0.48_{-0.87}^{+0.75}$ from Heymans et al. (2013). The baryon transfer function $F(k, z)$ is from Chisari et al. (2018); we use as fiducial values their Fig. 11, but randomly perturb $F(k, z)$ by $\delta F(k, z)=\delta \times(F(k, z)-1)$ with a normally distributed amplitude $\delta$ of the quoted RMS variance.

2016) ${ }^{7}$. For the fiducial redshift distribution of the lenses in (i), we take that of SM1, SM4, SM6, RED, and BLUE, and we then average the results. The redshift distribution of the sources are either BACK low- $z$ or BACK high- $z$.

We perform thousands of random variations in (i) to (iv) and average for all variations the quadratic means in the interval $1^{\prime} \lesssim \theta_{\text {ap }} \lesssim 2^{\circ}$ for $\delta b=\hat{b}_{2 \mathrm{D}} / \hat{b}_{2 \mathrm{D}}^{\mathrm{fid}}-1$ and $\delta r=\hat{r}_{2 \mathrm{D}} / \hat{r}_{2 \mathrm{D}}^{\mathrm{fid}}-1$ relative to our fiducial models $\hat{b}_{2 \mathrm{D}}^{\text {fid }}$ and $\hat{r}_{2 \mathrm{D}}^{\text {fid }}$ in Table 4 and the redshift distributions in Fig. 1. For every new variation in the cosmological model we draw a set of cosmological parameters from the published Planck MCMC posterior, thereby accounting for the correlations of their errors. Variations in the mean $(\delta z)$ and RMS width $(\delta \sigma)$ of redshift distributions of lenses and sources, the IA amplitude $\left(\delta A_{\mathrm{ia}}\right)$ and the baryon transfer function $(\delta F)$ are independently drawn from a Gaussian distribution with the variances given in Table 6 . To change the mean of a redshift distribution $p(z)$, we map $p(z) \rightarrow p(z[1+\delta z])$, whereas $p(z) \rightarrow p(z)^{1 /(1-\delta \sigma)^{2}}$ varies the width of the distribution (Sect. 7.3 in SH18). We perform this analysis for a scenario where all errors (i)-(iv) are present simultaneously but are statistically independent, and scenarios where only one error source (i)-(iv) is switched on at a time, giving the isolated RMS error $\sigma_{i}$. The detailed error distributions of the bias amplitudes can be found in Appendix F.

In summary, the RMS error $\sigma_{\text {tot }}$ for $b(k)$ ranges between $6.1 \%$ and $11.6 \%$, and between $4.3 \%$ and $6.9 \%$ for $r(k)$. For these values, the lower limits adopt uncorrelated systematic errors (i) to (iv) (the optimistic scenario, i.e. $\sigma_{\text {tot }}^{2}=\sum_{i} \sigma_{i}^{2}$ ), while the upper limits assume that systematic errors (i) to (iv) conspire to maximise the amplitude error (the conservative scenario, i.e. $\left.\sigma_{\text {tot }}=\sum_{i} \sigma_{i}\right)$.

However, these are not all the systematic errors that need to be considered. There may be additional systematic errors from the methodology in Sect. 4, for instance model bias by the templates or the likelihood, and its specific implementation in a

\footnotetext{
7 We use the MCMC posterior samples for base_w_plik HM_TTTEEE_lowTEB_BAO at http://pla.esac.esa.int/pla/ \#cosmology.
} 
computer code. According to SH18, these errors fall between $3 \%$ and $7 \%$ for $b(k)$ and between $3 \%$ and $5 \%$ for $r(k)$. These estimates were obtained from galaxy-bias reconstructions with mock lensing data (H15 based) that have exactly known galaxy bias, redshift distributions, and cosmological and IA parameters. Since they are related to methodology, they are uncorrelated to uncertainties in the galaxy bias normalisation.

Combining all the systematic errors above, the accuracy for $b(k)$ ranges somewhere between $6.8 \%$ (optimistic) and $13.5 \%$ (conservative), and for $r(k)$ between $5.2 \%$ and $8.5 \%$. A systematic error would be similar on all scales, and therefore offset the inferred biasing functions.

\section{Results}

\subsection{Biasing functions}

Our $b(k)$ and $r(k)$ results are shown on the following pages, after the references, as Figs. A.1-A.4 in the Appendix. The orange regions depict the $68 \%$ and $95 \%$ CIs of the reconstructed biasing functions around the median (dashed lines). The reconstructions assume the fiducial parameters in Table 4 and the galaxy redshift distributions in Fig. 1. There is one panel for each galaxy sample, indicated by the in-panel labels at the top left. For most CFHTLenS samples, the bias factor $b(k)$ clearly changes with $k$, while the high galaxy-matter correlation, $r(k) \approx 1$, has a moderate scale dependence.

The green data points show the SAM predictions in comparison: filled diamonds are for $\mathrm{H} 15$, and filled triangles are those for L12. In comparison to the models, the relative changes of the CFHTLenS $b(k)$ with $k$ is broadly similar to the models, but real galaxies are clearly more biased for $k \lesssim 0.5 h \mathrm{Mpc}^{-1}$. Towards the small spatial scales at higher $k$, the biasing of galaxies in both CFHTLenS and the models increases. Due to a steeper increase of L12 for SM2 to SM5, however, the Durham model typically agrees better with CFHTLenS than H15. Still, the models have a too small bias for SM1 and BLUE on all scales. With regard to the matter-galaxy correlation, the value of $r(k)$ evidently increases for $k \gtrsim 1 h \mathrm{Mpc}^{-1}$ in the CFHTLenS samples SM3 to SM6. This reflects, despite the noise, the scaledependence in the SAMs. The model agreement is somewhat worse for high- $z$ where the high value of $r(k)$ at $k \approx 10 \mathrm{~h} \mathrm{Mpc}^{-1}$ is not supported by the CFHTLenS samples SM1, SM2, RED, and BLUE.

For a test of signal robustness in our data we split the source catalogues into two subsamples of distinct mean redshifts $\bar{z}$ : BACK low $-z(\bar{z} \approx 0.97)$ into BACKa low $-z(\bar{z} \approx 0.86)$ and BACKb low $-z(\bar{z} \approx 1.12)$; BACK high $-z(\bar{z} \approx 1.03)$ into BACKa high $-z(\bar{z} \approx 0.93)$ and BACKb high $-z(\bar{z} \approx 1.14)$. Then we repeat every galaxy-bias reconstruction twice using those subsamples. The overlaid transparent grey regions in the figures are the $68 \%$ posterior CIs about the median for these reconstructions. Since they constrain the same biasing functions as the combined source sample, they should overlap with the filled darkorange region, and they should overlap with each other. This is indeed the case for most reconstructions. A few exceptions are

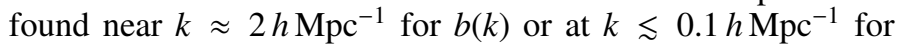
$r(k)$, such as for RED low- $z$ in both cases. Another disagreement is visible at small scales for SM4 high- $z$ where the two extra reconstructions prefer smaller $b(k)$ values. On the whole, however, and with some disagreement to be expected due to random chance, we consider the reconstruction overall robust. The next section tests the adequacy of the templates in describing the ratio statistics.

\subsection{Posterior predictive check of bias reconstruction}

A posterior predictive check validates the template models in our Bayesian deprojection (Gelman et al. 2003). We construct the posterior predictive, plotted in Figs. 5 and 6, by drawing values $\Theta \sim p\left(\boldsymbol{\Theta} \mid \boldsymbol{d}, \bar{n}_{\mathrm{g}}\right)$ from the posterior distribution of template parameters in Eq. (28) and by comparing the scatter of their $b_{2 \mathrm{D}}\left(\theta_{\mathrm{ap}} ; b\right)$ (left panels) and $r_{2 \mathrm{D}}\left(\theta_{\mathrm{ap}} ; b, r\right)$ (right panels) to the CFHTLenS data points of the ratio statistics. The ratio statistics is normalised by the prediction for unbiased galaxies using the fiducial cosmology in Table 4 . The $68 \%$ and $95 \%$ CIs are indicated by the shaded regions. We plot, for reference, $b_{2 \mathrm{D}}=r_{2 \mathrm{D}}=$ 1 as dotted lines. With the exception of $r_{2 \mathrm{D}}$ at $\theta_{\mathrm{ap}} \approx 6 \mathrm{arcmin}$ for high- $z$, the replicated and CFHTLenS data agree very well for the (normalised) ratio statistics $b_{2 \mathrm{D}}$ and $r_{2 \mathrm{D}}$. The origin of the $r_{2 \mathrm{D}}$ misfit is unclear, but it may be a bias of an overly smooth template or a systematic error in the shear data at this particular angular scale.

The projected galaxy bias in Figs. 5 and 6 nicely illustrates that the conflict with the SAM galaxy bias is already present before our deprojection of the ratio statistics; it has nothing to do with the specific templates in our analysis. For comparison with the CFHTLenS data points, we plot the normalised statistics and their $68 \%$ confidence intervals for the H15-based mock galaxy and shear catalogues without shape noise, used in SH18, as green solid lines (cosmological parameters as in the Millennium Simulation). Throughout, the $\mathrm{H} 15$ data for $b_{2 \mathrm{D}}$ are systematically lower than CFHTLenS on all angular scales. The correlation factor, $r_{2 \mathrm{D}}$, on the other hand, is broadly similar to CFHTLenS, with the notable exception of SM1 high-z. Similar data for L12 are not available to us. However, we do not expect their predictions to be significantly different to $\mathrm{H} 15$ due to their similar biasing functions for $k \lesssim 5 \mathrm{~h} \mathrm{Mpc}^{-1}$ (cf. green data points in Figs. A.1-A.4).

\subsection{Galaxy bias on linear scales}

Our technique separates galaxy bias in the non-linear (onehalo) regime from the linear stochastic bias $b_{\mathrm{ls}}$ and $r_{\mathrm{ls}}$ on large scales. The CFHTLenS results and model predictions are plotted in Fig. 7. Diamonds with error bars in the left panel are CFHTLenS posterior medians of $b_{\mathrm{ls}}$ with $68 \%$ CIs or $r_{\mathrm{ls}}$ in the right panel. Filled data points denote the low- $z$ samples and open data points the high- $z$ samples. The corresponding galaxy samples are shown at the bottom of each panel and the mean stellar masses at the top. The shaded rectangular regions indicate the $68 \%$ intervals of the average bias, obtained from merged samples SM1-SM6 in an extra analysis.

On the one hand, for $b_{\mathrm{ls}}$ there are clear differences from the model predictions, which are the green diamonds (H15) and triangles (L12): model galaxies are less clustered relative to matter. On the other hand, the relative bias $b_{i} / b_{j}$ for any two samples $i$ and $j$ is similar for CFHTLenS and the models. This can be seen when multiplying the H15 or L12 values by roughly 1.5 , bringing them into broad agreement with CFHTLenS. Furthermore, the models predict an evolution of biasing from low- $z$ (open symbols) to high- $z$ (filled symbols) that is too small for the CFHTLenS errors. Nevertheless, our merged samples SM1-SM6 weakly indicate an evolution but with the opposite trend to in the models, namely a decline in $b_{1 \mathrm{~s}}\left(68 \%\right.$ credibility) from $1.98_{-0.18}^{+0.20}$ at low- $z$ to $1.65_{-0.12}^{+0.17}$ at high- $z$.

The blue squares in the left panel are non-lensing measurements of $b_{\mathrm{ls}}$ by Marulli et al. (2013) in the VIMOS Public Extragalactic Redshift Survey (VIPERS) for galaxy samples similar to 
Posterior predictive vs. data

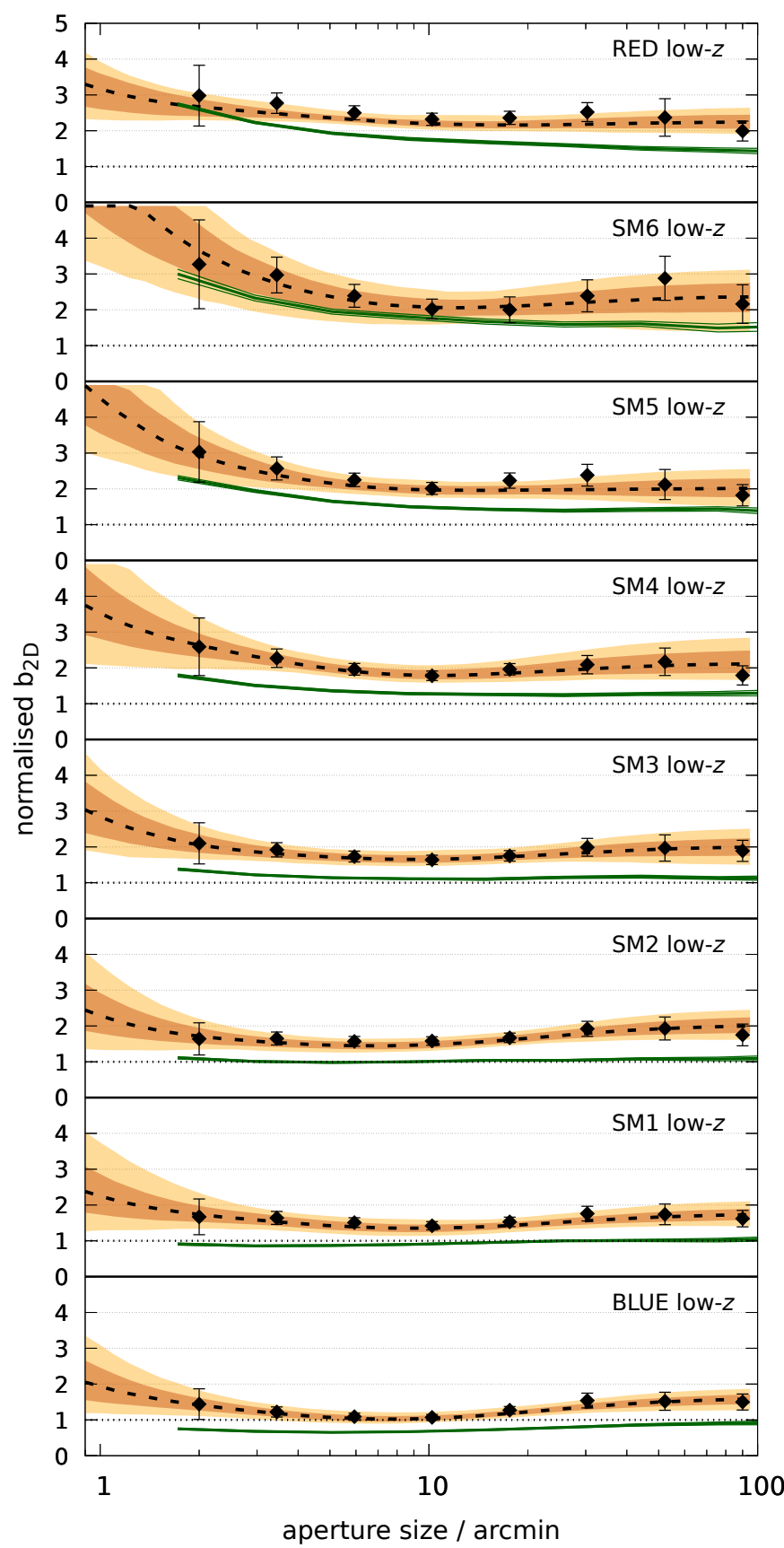

Posterior predictive vs. data

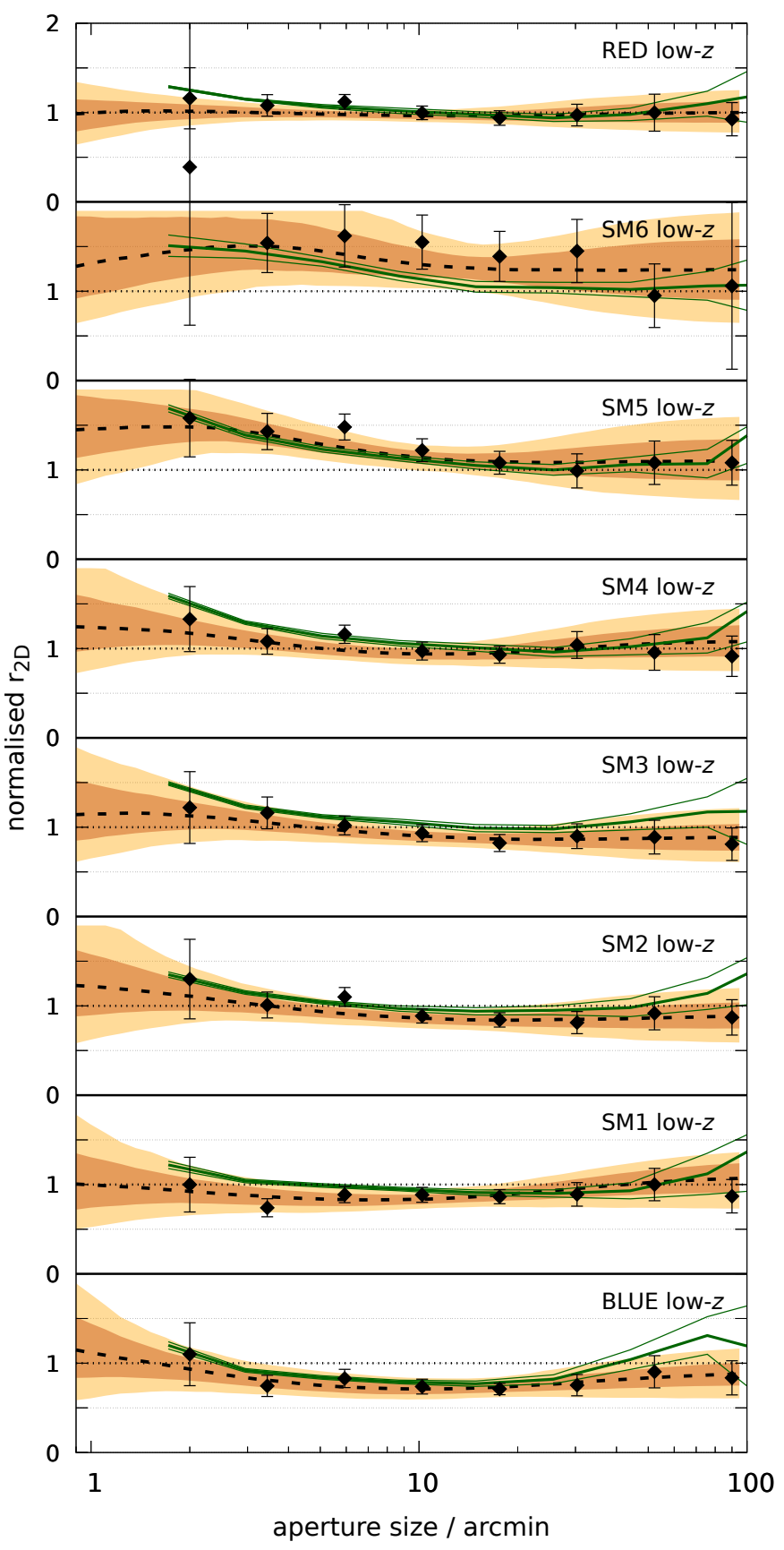

Fig. 5. Posterior predictive checks for the low-z CFHTLenS samples. The data points are normalised bias measurements $b_{2 \mathrm{D}}\left(\right.$ left panel) and $r_{2 \mathrm{D}}$ (right panel) with jackknife errors as a function of aperture size. The orange regions are the posterior predictive distributions of our templates with $68 \%$ and $95 \%$ CIs; the dashed lines are the median. The green solid lines depict the normalised bias and 68\% confidence intervals for H15 mock data in SH18 without shape noise.

ours at high- $z$ (Sect. 2.4). For their estimate of $b_{\mathrm{ls}}$, the VIPERS samples were divided into three stellar-mass bins, encompassing $10^{9.8} h_{70}^{-2} \mathrm{M}_{\odot}$ to $10^{11.8} h_{70}^{-2} \mathrm{M}_{\odot}$, and the galaxy-clustering correlation function between 1 and $10 h^{-1} \mathrm{Mpc}$ was normalised by the theoretical clustering of dark matter for a flat $\Lambda \mathrm{CDM}$ universe $\left(\Omega_{\mathrm{m}}=0.25, \sigma_{8}=0.8, n_{\mathrm{s}}=1\right)$. To make these results comparable to ours, we interpolate the VIPERS data points (their Table 4) to the mean stellar masses of SM2 to SM6 by using a best-fitting second-order polynomial. Then, except for our higher SM2 data point, the VIPERS results agree well with our lensing-based high- $z$ measurements (open diamonds) and conflict with the SAMs for the highest stellar masses SM5 and SM6.

The linear correlation factors in the right panel are $68 \%$ consistent with a deterministic bias $r_{\mathrm{ls}}=1$ (and the SAMs), except for two cases: BLUE and, in particular, SM1 high- $z$ significantly fall below this value. Since SM1 high- $z$ is an interesting case, Fig. 8 (right panel) plots its $\hat{r}_{2 \mathrm{D}}$ as function of aperture size. The lines are predictions for unbiased galaxies with IA amplitudes varying between $-3 \leq A_{\mathrm{ia}} \leq 3$. Importantly, all CFHTLenS galaxies with $r_{\mathrm{ls}}=1$, bias or unbiased, should for large apertures be consistent with our fiducial value $A_{\mathrm{ia}}=-0.48$. 
Posterior predictive vs. data

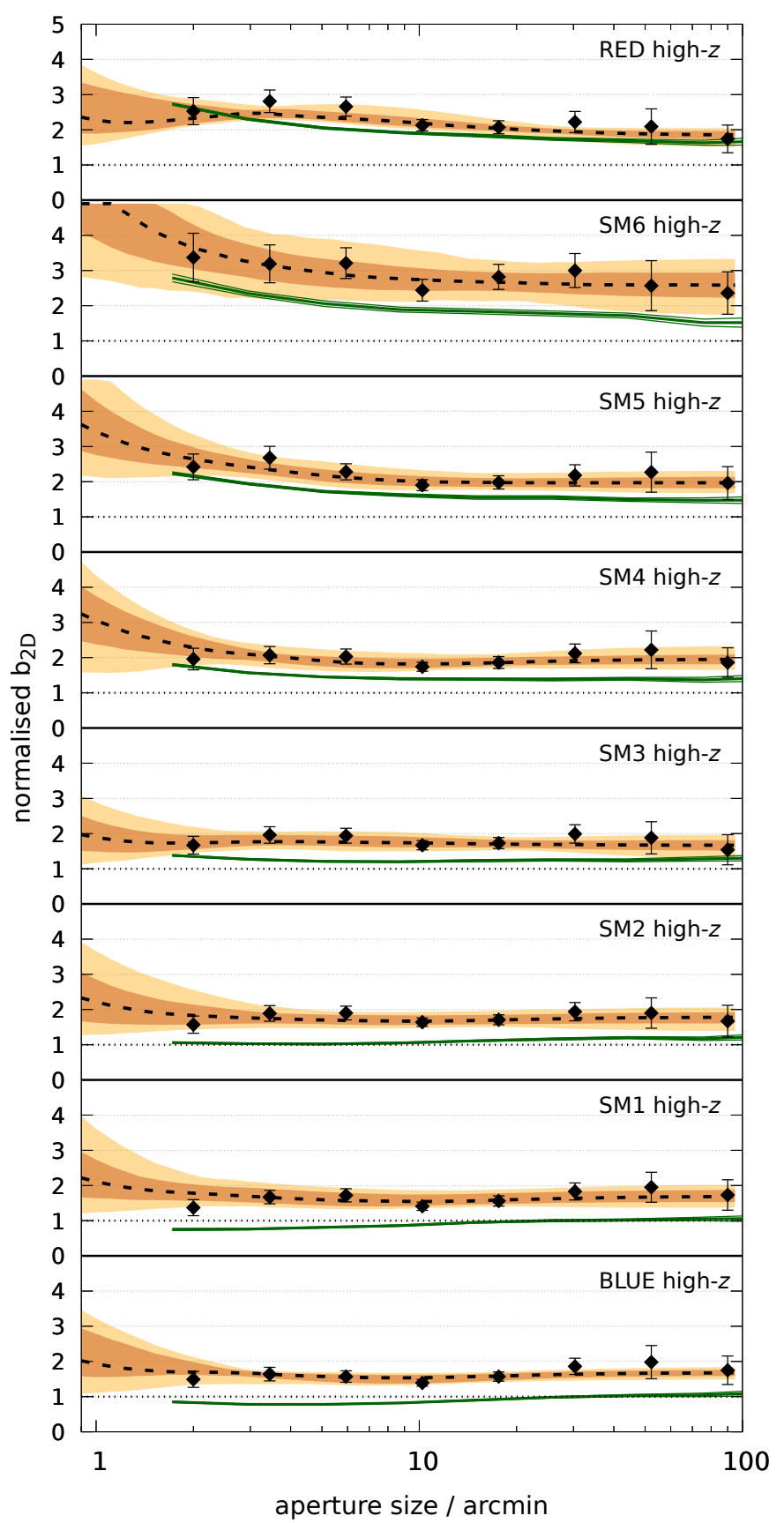

Posterior predictive vs. data

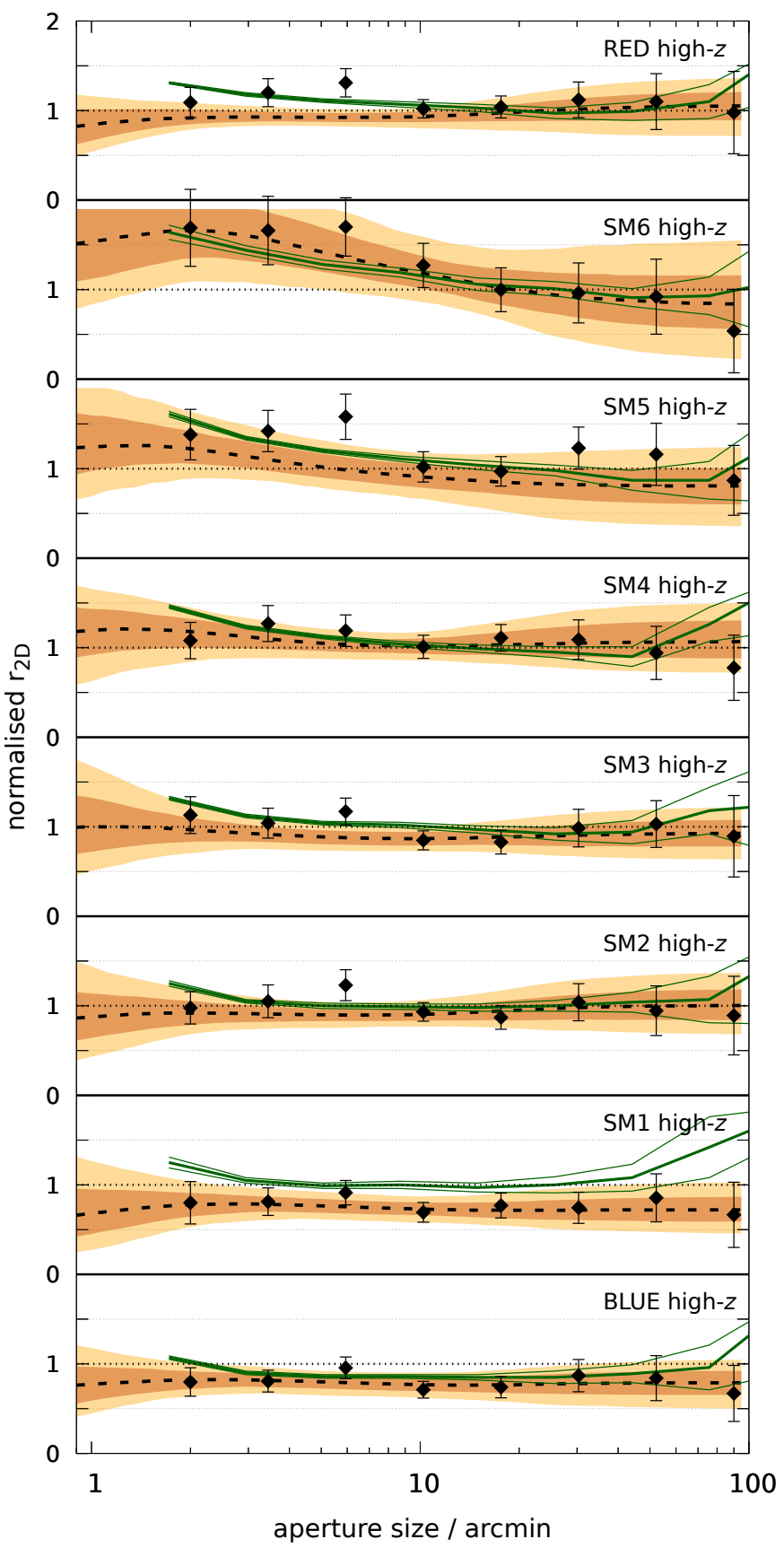

Fig. 6. As in Fig. 5, but for the high- $z$ samples. There are signs of a model misfit to $r_{2 \mathrm{D}}$ at around 6 arcmin, especially for RED and SM5. There is also weak evidence in favour of a stochastic bias, $r_{2 \mathrm{D}}<1$, for SM1 and BLUE at degree scales (bottom right panels).

While this is clearly the case for SM1 low-z (left panel), the data points of SM1 high- $z$ in the right panel fall even below $A_{\mathrm{ia}}=-3$ for $\theta_{\mathrm{ap}} \gtrsim 30$ arcmin, reflecting our low value for $r_{\mathrm{ls}}$ in the Bayesian interpretation of the aperture statistics.

\subsection{Template parameters}

Our main results are the reconstructed biasing functions for CFHTLenS in Figs. A.1-A.4. Nevertheless, marginal constraints for the template parameters in the one-halo regime (i.e. $b(m)$, $V(m), f_{\text {cen }}, \zeta$, and $\left.m_{\text {piv }}\right)$ are also reported in Appendix $G$ and are briefly summarised here. These inform us about physical galaxy parameters, albeit with significantly lower accuracy compared to the reconstructed $b(k), r(k)$, and their asymptotic values $b_{\mathrm{ls}}$ and $r_{\mathrm{ls}}$ on large scales.

Typically, we find only weak constraints for HOD-related template parameters in the CFHTLenS data, yet differences to the SAMs are visible for $b(m)$ : CFHTLenS galaxies of same stellar mass seemingly prefer to populate halos of higher mass when compared to the true $b(m)$ of the SAMs. However, this shift of the inferred CFHTLenS $b(m)$ towards higher halo masses is at least partly an artefact of the crude template modelling because it is also visible in the simulated reconstruction of biasing functions in SH18, using H15-based mock data. We also note here that the specific results for $b(m)$ (and $V(m)$ ) rely on the adopted 

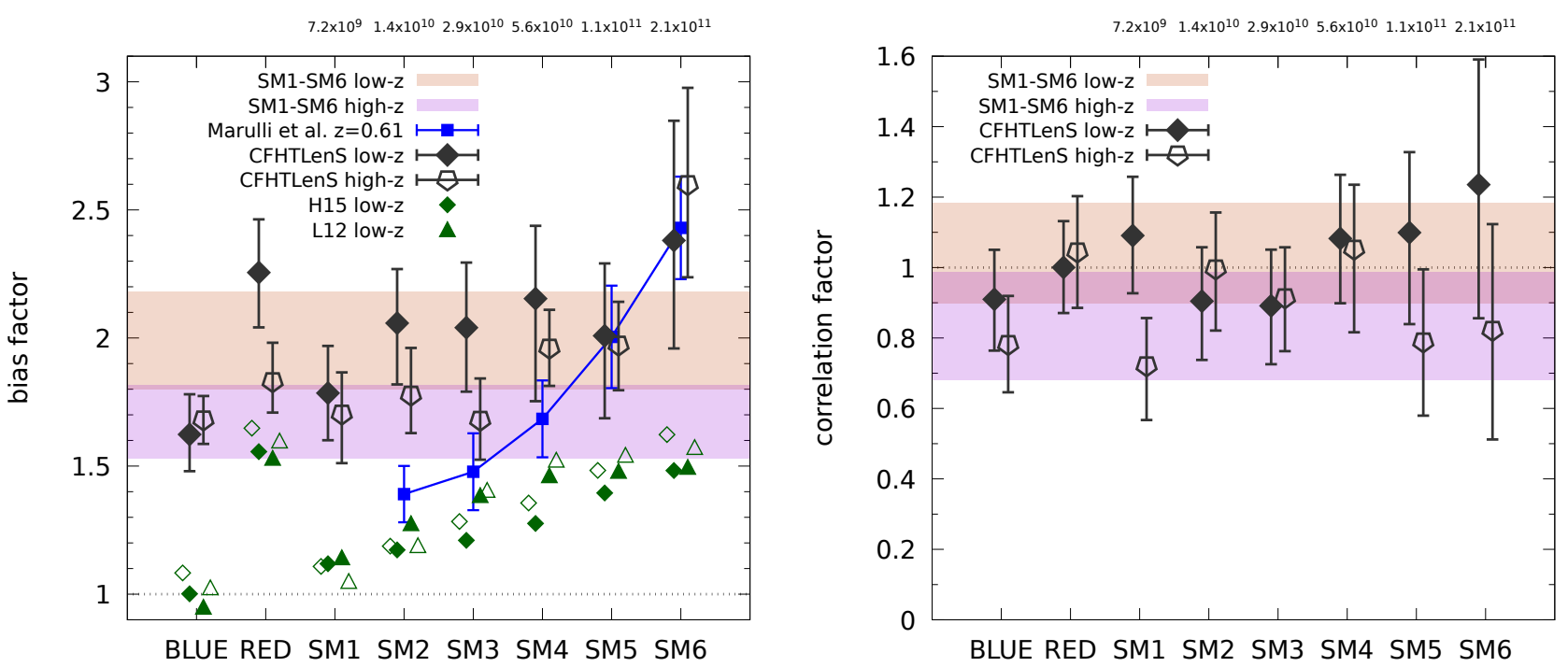

Fig. 7. Large-scale bias $b_{\mathrm{ls}}$ (left panel) and correlation $r_{\mathrm{ls}}$ (right panel) for the galaxy populations indicated on the $x$-axis. The black data points are CFHTLenS results (medians and 68\% CIs), the green data points in the left panel are SAM predictions (diamonds for H15, triangles for L12, filled symbols for low- $z$, and open symbols for high- $z$ ). The shaded areas are 68\% CIs for CFHTLenS, merging SM1 to SM6 in an extra analysis. The blue squares are VIPERS $b_{1 \mathrm{~s}}$ for stellar-mass binned galaxies with $i_{\mathrm{AB}}<22.5$ and median $\bar{z}=0.61$ by Marulli et al. (2013).
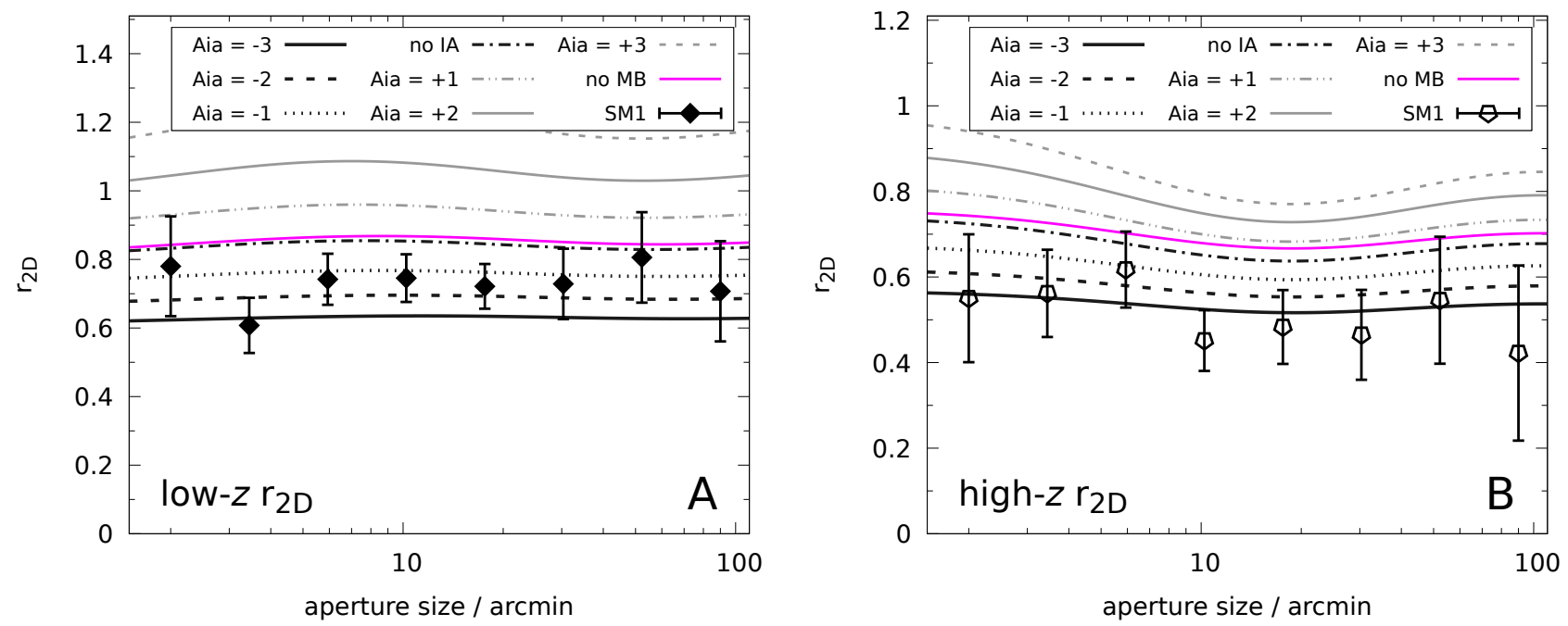

Fig. 8. Ratio statistics $\hat{r}_{2 \mathrm{D}}$ as a function of aperture size. The data points with error bars (jackknife 68\% errors) are for SM1 low- $z$ (left panel $A$ ) and SM1 high- $z$ (right panel $B$ ). The lines are predictions for unbiased galaxies with varying values of $A_{\mathrm{ia}} \in[-3,+3]$. Our fiducial value is $A_{\mathrm{ia}}=-0.48$. The solid magenta line 'no MB' is a model for $A_{\mathrm{ia}}=0$ and no magnification.

halo-mass spectrum $n(m)$ which differs for CFHTLenS and the Millennium Simulation due to different fiducial cosmologies. Furthermore, the normalised excess variance $V(m)$ is mostly consistent with a Poisson variance of galaxy numbers inside halos $(V(m)=0)$ or possibly a sub-Poisson variance $(V(m)<0)$ around $10^{13} \lesssim m_{\text {piv }} \lesssim 10^{14} h^{-1} \mathrm{M}_{\odot}$ in some cases, SM6 high- $z$ for instance. In comparison, the $V(m)$ for the SAMs also indicates a sub-Poisson variance but again offset to somewhat lower halo masses. For high halo masses $m \gtrsim 10^{14} h^{-1} \mathrm{M}_{\odot}$ and stellar masses below SM5 the variance of galaxy numbers $N(m)$ becomes super-Poisson $(V(m)>0)$ for H15. This is neither seen in L12 nor visible in our CFHTLenS results. Then the distribution of satellites is consistent with that of matter inside halos $(\zeta \sim 1)$ within typical errors of roundabout \pm 0.45 (68\% CI). And, finally, the mixing parameter of templates with and without central galaxies, $f_{\text {cen }}$, falls broadly within $20 \%$ and $80 \%$, including the high values of $70 \% \lesssim f_{\text {cen }} \lesssim 90 \%$ in the SAMs.

\section{Discussion}

Our results for the galaxy bias in Figs. 7 and A.1-A.4 (Appendix) clearly show a conflict with the SAM predictions by H15 (Munich model) and L12 (Durham model). This conflict hints at inaccuracies in the SAM modelling of galaxy physics through its connection with galaxy bias (e.g. Weinberg et al. 2004; Blanton et al. 1999). There is no clear preference for any model by CFHTLenS, despite the inter-model differences on small scales, most prominent for $b(k)$ in SM3 to SM5. We discuss our findings and possible systematic errors below.

The linear bias factor $b_{\mathrm{ls}}$ is overall $50 \%$ higher relative to the models due to a stronger clustering of galaxies in CFHTLenS. This may highlight modelling problems for $z \gtrsim 0.3$ that affect all stellar masses. We measure for $b(k)$ and $b_{1 \mathrm{~s}}$ the amplitude ratio of galaxy and matter clustering in the data. Comparing the amplitudes of $\left\langle\mathcal{N}^{2}\right\rangle$ in CFHTLenS on scales $4^{\prime} \leq \theta_{\text {ap }} \leq$ 


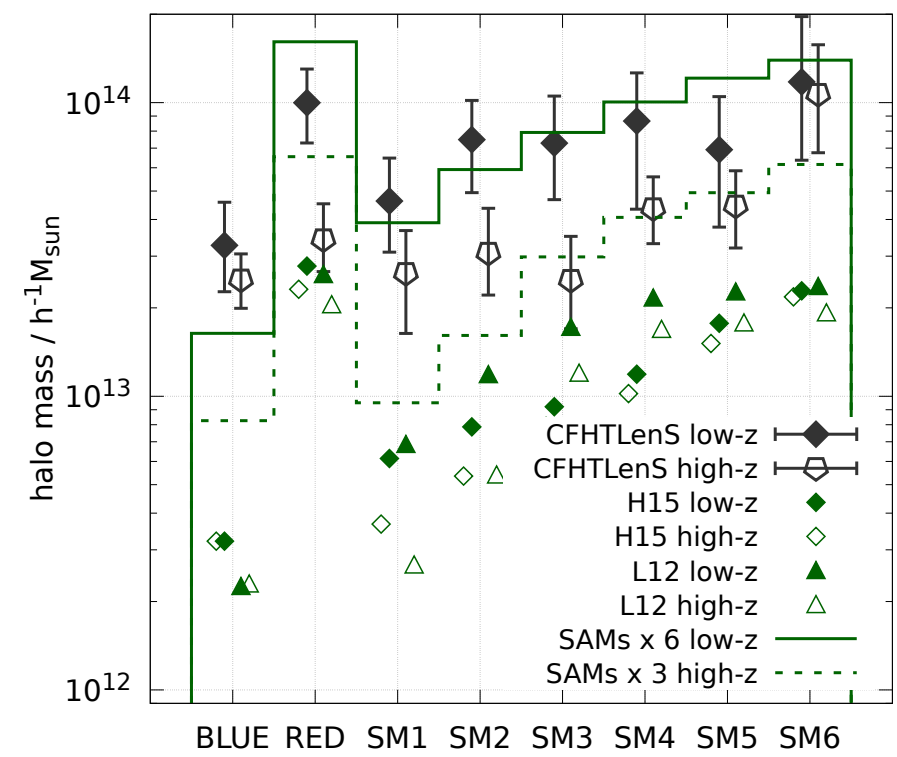

Fig. 9. Characteristic halo mass $m_{*}$ that corresponds to the large-scale bias $b_{\mathrm{ls}}$ in Fig. 7 for the halo bias model by Tinker et al. (2005) and the Millennium Simulation cosmology as reference (Sect. 3.1). Data points with error bars indicate the CFHTLenS galaxy samples ( $x$-axis) and the green points are the SAM predictions. The green lines average the SAM values and rescale them by a factor of 6 (solid line for low-z) or 3 (dashed line for high-z).

$40^{\prime}$ to the H15 mock data in SH18, we find typical values in CFHTLenS that are $20 \%$ to $40 \%$ higher. This conflict is further increased in $b_{\mathrm{ls}}$ by a weaker clustering of matter in CFHTLenS, $\Sigma_{8}=\sigma_{8} \sqrt{\Omega_{\mathrm{m}} / 0.27} \approx 0.76$, relative to that in the Millennium Simulation, $\Sigma_{8} \approx 0.87$. Problems with galaxy clustering in the Durham and Munich models are known for low-mass galaxies at $z \lesssim 0.1$ on small scales of $\sim 1 h^{-1} \mathrm{Mpc}$ (Henriques et al. 2017; van Daalen et al. 2016; Farrow et al. 2015; Kang 2014). According to Fig. 7, supported by the VIPERS data for SM3SM6, this problem deepens in the range $0.3 \lesssim z \lesssim 0.6$ and then also affects linear scales and stellar masses as high as $M_{*} \sim 10^{11} h_{70}^{-2} \mathrm{M}_{\odot}$. In a halo-model picture the lower $b_{\mathrm{ls}}$ suggests that $\mathrm{H} 15$ or L12 galaxies tend to reside inside matter halos of systematically low mass, decreasing the clustering amplitude on linear scales (Berlind \& Weinberg 2002). At first sight this seems to be supported by the constraints of our template parameters for $b(m) \propto\langle N \mid m\rangle / m$ (Fig. G.2), but we caution here that the templates, which are employed for a different purpose, have not been tested for their ability to truthfully reconstruct $b(m)$. Clearly visible in Fig. 2, however, is that the SMF of the SAMs in the two redshift bins closely matches that in CFHTLenS. First of all, this supports a selection of galaxy populations compatible with CFHTLenS; secondly, if there are differences in the SAMs' $\langle N \mid m\rangle$, then they have to be such that we nevertheless obtain similar galaxy number densities $\bar{n}_{\mathrm{g}}=$ $\int_{0}^{\infty} \mathrm{d} m n(m)\langle N \mid m\rangle$. In other words, variations $\delta\langle N \mid m\rangle$ between the SAMs and CFHTLenS have to satisfy $\int_{0}^{\infty} \mathrm{d} m n(m) \delta\langle N \mid m\rangle \approx$ 0 . The large-scale bias factor, on the other hand, can be changed by $\delta b_{\mathrm{ls}}=\bar{n}_{\mathrm{g}}^{-1} \int_{0}^{\infty} \mathrm{d} m n(m) b_{\mathrm{h}}(m) \delta\langle N \mid m\rangle$ while keeping $\bar{n}_{\mathrm{g}}$ fixed. To identify HOD variations between CFHTLenS and the SAMs, permitted by this $\delta\langle N \mid m\rangle$ constraint, let us crudely assume that all galaxies in a sample live inside halos of characteristic mass $m_{*}$, hence exhibiting the large-scale bias $b_{\mathrm{ls}}=b_{\mathrm{h}}\left(m_{*}\right)$. In Fig. 9, we explore the relative $m_{*}$ differences needed to explain the $b_{\mathrm{ls}}$ of observational data and models, using the same reference cosmology for both to make them comparable (see that of the Millennium Simulation, Sect. 3.1). For the halo bias factor $b_{\mathrm{h}}(m)$, we employ Tinker et al. (2005) at the mean redshift of a galaxy sample. On average, the $m_{*}$ in CFHTLenS is broadly a factor of 6(3) higher than the average SAM at low $-z$ (high- $z$ ), as indicated by the solid (dashed) line; in the SAMs $m_{*}$ increases from around $4 \times 10^{12} h^{-1} \mathrm{M}_{\odot}$ for SM1 to $2 \times 10^{13} h^{-1} \mathrm{M}_{\odot}$ for SM6. In conclusion, our results hint at problems in H15 and L12 for $z \gtrsim 0.3$. These problems may be related to inaccuracies in their HOD, preferring parent halos of 3 to 6 times lower mass than in reality. This view, however, assumes well-controlled systematic errors in our galaxy selection and the reconstruction of galaxy bias.

In this respect it is conceivable that the conflicting results might also indicate large systematic errors in our galaxy bias amplitude combined with an inconsistent selection of SAM galaxies, such as systematically low CFHTLenS stellar masses. The estimated systematic RMS error in $b_{\mathrm{ls}}$ is somewhere within $\left[6.8 \%, 13.5 \%\right.$ ] (Sect. 4.9). This is well below the $b_{\text {ls }}$ conflict of $\sim 50 \%$, and therefore cosmology or redshift errors alone probably do not explain the model conflict. But two other systematic errors in the galaxy sample selection can also bias our results. Firstly, our flux limit of $i^{\prime}<22.5$ might remove too many faint weakly clustered galaxies in CFHTLenS if the SAM $i^{\prime}$-band magnitudes are too faint relative to CFHTLenS (the flux limit is also applied to the SAMs). Even then the SAM conflict for the massive (luminous) galaxies $M_{*} \gtrsim 10^{11} h_{70}^{-2} \mathrm{M}_{\odot}$ is hard to explain where the flux limit is irrelevant. In addition, the SAM luminosity functions give good fits to observations for $z \lesssim 1$ (e.g. Bower et al. 2006); the Durham model even uses luminosity functions in two bands for the model calibration. We note that the low-mass samples SM1 and SM2 are especially affected by the flux limit, implying a higher value for $b_{\mathrm{ls}}$ compared to volume-limited stellar-mass samples because the bias factor increases with galaxy luminosity (e.g. Zehavi et al. 2005; Norberg et al. 2002). More relevant could be a second selection bias: stellar-mass estimates might be systematically low in CFHTLenS. Shifting the data points in the left panel of Fig. 7 by $\Delta \log _{10} M_{*}=+0.6$ dex gives a better match to the SAM predictions for $b_{1 \mathrm{~s}}$ (e.g. SM1 to SM3, SM2 to SM4). On the other hand, a stellar-mass error that large is hard to justify considering previous error estimates (Sect. 2.1 of Velander et al. 2014), and considering that the VIPERS results for both $b_{\mathrm{ls}}$ and $\bar{n}_{\mathrm{g}}$, using full spectroscopic information, are comparable to ours in the high- $z$ bin (Marulli et al. in Fig. 7; Davidzon et al. in Fig. 2). In addition, the stellar-mass functions of the SAMs and CFHTLenS in Fig. 2 are good matches, which is unlikely for systematically different CFHTLenS stellar masses. Nevertheless, a large yet realistic stellar-mass error of $\sim 0.3$ dex combined with a very large systematic error of $\sim 25 \%$ for $b_{\text {ls }}$ could in principle resolve the SAM conflict for the large-scale galaxy clustering. A systematic error of such magnitude in $b_{\mathrm{ls}}$, however, would imply that all our results for $r_{\mathrm{ls}} \approx 1$ in Fig. 7 have to be offset by about $15 \%$, which would then mildly conflict with a deterministic galaxy bias on large scales; systematic errors in $r_{\mathrm{ls}}$ are typically $50 \%$ to $70 \%$ of those in $b_{1 \mathrm{~s}}$ (Appendix F).

For systematic errors within the estimated range, our results for $r_{\mathrm{ls}}$ agree with the SAM picture of a deterministic galaxy bias. Only the high- $z$ samples SM1 and BLUE are weak evidence for a stochastic linear bias, which will be tested with upcoming survey data. Although the biasing functions $b(k)$ and $r(k)$ generally do not distinguish non-linear deterministic bias from a stochastic bias, the linear regime is an exception, where 
$\tilde{\delta}_{\mathrm{g}} \approx b_{\mathrm{ls}} \tilde{\delta}_{\mathrm{m}}+\epsilon$ and $r_{\mathrm{ls}}$ is only sensitive to the shot-noise corrected stochastic scatter $\epsilon$ (Tegmark \& Peebles 1998; Dekel \& Lahav 1999). For this regime, and after the IA (and magnification) correction, the data points in the right panel of Fig. 7 establish, in agreement with the SAMs, a deterministic bias $r_{\mathrm{ls}}=1$ for CFHTLenS galaxies on linear scales. More interestingly, and contrary to the SAMs, the samples SM1 and BLUE high- $z$ prefer an average stochastic bias $r_{\mathrm{ls}} \approx 0.75 \pm 0.14$ (stat.) \pm 0.06 (sys.) at $68 \%$ credibility. Some stochasticity of blue galaxies, $r \approx 0.9$ on scales of $15 h^{-1} \mathrm{Mpc}$, is supported by other observations, but it is unclear if this is still present on linear scales and for our redshift regime (Patej \& Eisenstein 2016; Swanson et al. 2008; Wild et al. 2005; Blanton 2000). At least for the CMB lensing results of $i$-band limited galaxies by Giannantonio et al. (2016), a linear stochastic bias is one interpretation (Sect. 7.4). Unfortunately, our new evidence for stochasticity $(2 \sigma)$ is further weakened by the systematic amplitude uncertainty of [5.2\%, 8.5\%], quoted above as 'sys.' for the pessimistic $8.5 \%$ (Sect. 4.9). In the near future we anticipate better constraints from the Kilo Degree Survey (de Jong et al. 2013), the Hyper SuprimeCam Survey (Aihara et al. 2018), or the Dark Energy Survey (Abbott et al. 2016) which will have ten times the survey area of CFHTLenS and a better control of galaxy redshift distributions, which are, in addition to IA, the major source for the systematic error in $r_{\mathrm{ls}}$ (bottom panels in Fig. F.1).

Turning to the non-linear regime, CFHTLenS galaxy biasing clearly varies with scale, stellar mass, and colour, but differently from the SAMs and with no clear preference for either H15 or L12. Nevertheless, there is a qualitative agreement with the models insofar as the bias factor $b(k)$ increases on non-linear scales $k \sim 10 \mathrm{~h} \mathrm{Mpc}^{-1}$ relative to linear scales by up to a factor of two (Figs. A.1 and A.2). The relative increase of $r(k)$ is weaker, typically a factor of 1.2 (Figs. A.3 and A.4). These relative changes are insensitive to systematic errors in our method which offset the biasing functions as a whole. Physically the $b(k)$ increase is probably related to central galaxies inside halos that have $\langle N \mid m\rangle \lesssim 1$ and dominate the signal for sufficiently large $k$ (Sect. 5.3 in SH18). Central galaxies affect $r(k)$ in a similar way, like probably the stellar mass samples of CFHTLenS at low $-z$. For other samples, however, where the $r(k)$ increase is small or absent in contrast to the SAMs, this interpretation is less clear, RED and BLUE high- $z$ for instance. For a physical interpretation of these observations, an accurate halo-model fit (e.g. Dvornik et al. 2018; Seljak et al. 2005) or a study of $b(k)$ and $r(k)$ variations with respect to SAM parameters is needed.

Finally, in a broader context, the possibility of a stochastic galaxy bias, IA distortions, or magnification effects on linear scales is also relevant for the popular cosmological probe $E_{\mathrm{G}}$ for gravity (Reyes et al. 2010). This probe relies on a deterministic bias, whereas a stochastic bias changes the value of $E_{\mathrm{G}}$ to $r_{\mathrm{ls}} E_{\mathrm{G}}$. Additionally, lensing magnification changes $E_{\mathrm{G}}$ through the galaxy-galaxy lensing (GGL) amplitude by up to several per cent, depending on type and mean redshift of lens galaxies (Fig. 4 in SH18). Likewise, GI correlations bias the GGL signal if the distributions of lenses and sources overlap in radial direction (Hirata \& Seljak 2004). SH18 estimate the GI bias of GGL, and thus of $E_{\mathrm{G}}$, to up to $5 \%$ for $-2 \lesssim A_{\text {ia }} \lesssim-1$ and lens, source samples similar to ours (Fig. 3 in SH18). While this GI contamination can in principle be reduced to zero by avoiding any overlap, the magnification bias is always present and has to be accounted for by applying the method in Unruh et al. (2020), for example. Importantly, our work suggests not to use BLUE (star-forming) or SM1-like galaxies as lenses for $E_{\mathrm{G}}$ due to the possibility of a stochastic bias.
At the CDS, we provide Monte Carlo realisations of the posterior distributions of $b(k)$ and $r(k)$ for every galaxy sample in our analysis. The scatter among the realisations reflects the distribution of their statistical errors, and the full set of realisations can be utilised to propagate this uncertainty in future studies.

Acknowledgements. We thank Christopher Bonnett for fruitful discussions at an early stage of this project, and we thank Peter Schneider and Catherine Heymans for comments on the paper manuscript. This work has been supported by Collaborative Research Center TR33 'The Dark Universe' and by the Deutsche Forschungsgemeinschaft through the project SI 1769/1-1. This work is based on observations obtained with MegaPrime/MegaCam, a joint project of CFHT and CEA/DAPNIA, at the Canada-France-Hawaii Telescope (CFHT) which is operated by the National Research Council (NRC) of Canada, the Institut National des Sciences de l'Univers of the Centre National de la Recherche Scientifique (CNRS) of France, and the University of Hawaii. This research used the facilities of the Canadian Astronomy Data Centre operated by the National Research Council of Canada with the support of the Canadian Space Agency. CFHTLenS data processing was made possible thanks to significant computing support from the NSERC Research Tools and Instruments grant program.

\section{References}

Abbott, T., Abdalla, F. B., Allam, S., et al. 2016, Phys. Rev. D, 94, 022001 Aihara, H., Arimoto, N., Armstrong, R., et al. 2018, PASJ, 70, S4 Baldry, I. K., Driver, S. P., Loveday, J., et al. 2012, MNRAS, 421, 621 Benítez, N. 2000, ApJ, 536, 571

Benjamin, J., Van Waerbeke, L., Heymans, C., et al. 2013, MNRAS, 431, 1547 Berlind, A. A., \& Weinberg, D. H. 2002, ApJ, 575, 587

Bertin, E., \& Arnouts, S. 1996, A\&AS, 117, 393

Bielby, R., Hudelot, P., McCracken, H. J., et al. 2012, A\&A, 545, A23 Blanton, M. 2000, ApJ, 544, 63

Blanton, M., Cen, R., Ostriker, J. P., \& Strauss, M. A. 1999, ApJ, 522, 590

Bolzonella, M., Kovač, K., Pozzetti, L., et al. 2010, A\&A, 524, A76

Bolzonella, M., Miralles, J. M., \& Pelló, R. 2000, A\&A, 363, 476

Bower, R. G., Benson, A. J., Malbon, R., et al. 2006, MNRAS, 370, 645

Bridle, S., \& King, L. 2007, New J. Phys., 9, 444

Bruzual, G., \& Charlot, S. 2003, MNRAS, 344, 1000

Cacciato, M., Lahav, O., van den Bosch, F. C., Hoekstra, H., \& Dekel, A. 2012, MNRAS, 426, 566

Cacciato, M., van den Bosch, F. C., More, S., Mo, H., \& Yang, X. 2013, MNRAS, 430, 767

Calzetti, D., Armus, L., Bohlin, R. C., et al. 2000, ApJ, 533, 682

Campbell, D., van den Bosch, F. C., Padmanabhan, N., et al. 2018, MNRAS, 477,359

Chabrier, G. 2003, PASP, 115, 763

Chisari, N. E., Richardson, M. L. A., Devriendt, J., et al. 2018, MNRAS, 480, 3962

Cole, S., Lacey, C. G., Baugh, C. M., \& Frenk, C. S. 2000, MNRAS, 319, 168 Comparat, J., Jullo, E., Kneib, J.-P., et al. 2013, MNRAS, 433, 1146

Contreras, S., Baugh, C. M., Norberg, P., \& Padilla, N. 2013, MNRAS, 432, 2717

Cooray, A., \& Sheth, R. 2002, Phys. Rep., 372, 1

Davidzon, I., Bolzonella, M., Coupon, J., et al. 2013, A\&A, 558, A23

de Jong, J. T. A., Verdoes Kleijn, G. A., Kuijken, K. H., \& Valentijn, E. A. 2013, Exp. Astron., 35, 25

Dekel, A., \& Lahav, O. 1999, ApJ, 520, 24

Desjacques, V., Jeong, D., \& Schmidt, F. 2018, Phys. Rep., 733, 1

Di Porto, C., Branchini, E., Bel, J., et al. 2016, A\&A, 594, A62

Dvornik, A., Hoekstra, H., Kuijken, K., et al. 2018, MNRAS, 479, 1240

Erben, T., Hildebrandt, H., Miller, L., et al. 2013, MNRAS, 433, 2545

Farrow, D. J., Cole, S., Norberg, P., et al. 2015, MNRAS, 454, 2120

Friedrich, O., Gruen, D., DeRose, J., et al. 2018, Phys. Rev. D, 98, 023508

Fu, L., Kilbinger, M., Erben, T., et al. 2014, MNRAS, 441, 2725

Gelman, A., Carlin, J., Stern, H., \& Rubin, D. 2003, Bayesian Data Analysis,

Second Edition, Chapman \& Hall/CRC Texts in Statistical Science (Taylor \& Francis)

Giannantonio, T., Fosalba, P., Cawthon, R., et al. 2016, MNRAS, 456, 3213

Gruen, D., Friedrich, O., Krause, E., et al. 2018, Phys. Rev. D, 98, 023507

Guzik, J., \& Seljak, U. 2001, MNRAS, 321, 439

Hartlap, J. 2009, http://hss.ulb.uni-bonn.de/2009/1796/1796.htm $\mathrm{PhD}$ Thesis, AIfA - Argelander-Institut für Astronomie, Rheinische Friedrich-Wilhelms Universität Bonn, Germany

Henriques, B. M. B., White, S. D. M., Thomas, P. A., et al. 2015, MNRAS, 451, 2663 
Henriques, B. M. B., White, S. D. M., Thomas, P. A., et al. 2017, MNRAS, 469, 2626

Heymans, C., Grocutt, E., Heavens, A., et al. 2013, MNRAS, 432, 2433

Heymans, C., Van Waerbeke, L., Miller, L., et al. 2012, MNRAS, 427, 146

Heymans, C., Tröster, T., Asgari, M., et al. 2021, A\&A, in press, https: //doi . org $/ 10.1051 / 0004-6361 / 202039063$

Hilbert, S., Hartlap, J., White, S. D. M., \& Schneider, P. 2009, A\&A, 499, 31

Hildebrandt, H., Erben, T., Kuijken, K., et al. 2012, MNRAS, 421, 2355

Hirata, C. M., \& Seljak, U. 2004, Phys. Rev. D, 70, 063526

Hoekstra, H., Yee, H. K. C., \& Gladders, M. D. 2001, ApJ, 558, L11

Hoekstra, H., van Waerbeke, L., Gladders, M. D., Mellier, Y., \& Yee, H. K. C. 2002, ApJ, 577, 604

Joachimi, B., Mandelbaum, R., Abdalla, F. B., \& Bridle, S. L. 2011, A\&A, 527, A26

Joudaki, S., Blake, C., Heymans, C., et al. 2017, MNRAS, 465, 2033

Jullo, E., Rhodes, J., Kiessling, A., et al. 2012, ApJ, 750, 37

Kang, X. 2014, MNRAS, 437, 3385

Kilbinger, M., Benabed, K., Guy, J., et al. 2009, A\&A, 497, 677

Kilbinger, M., Bonnett, C., \& Coupon, J. 2014, athena: Tree code for secondorder correlation functions (Astrophysics Source Code Library)

Knight, K. 1999, Mathematical Statistics, Chapman \& Hall/CRC Texts in Statistical Science (CRC Press)

Kovač, K., Porciani, C., Lilly, S. J., et al. 2011, ApJ, 731, 102

Lacey, C. G., Baugh, C. M., Frenk, C. S., et al. 2016, MNRAS, 462, 3854

Lagos, C. D. P., Bayet, E., Baugh, C. M., et al. 2012, MNRAS, 426, 2142

Marulli, F., Bolzonella, M., Branchini, E., et al. 2013, A\&A, 557, A17

Miller, L., Heymans, C., Kitching, T. D., et al. 2013, MNRAS, 429, 2858

Mo, H., van den Bosch, F. C., \& White, S. 2010, Galaxy Formation and Evolution (UK: Cambridge University Press)

Navarro, J. F., Frenk, C. S., \& White, S. D. M. 1996, ApJ, 462, 563

Norberg, P., Baugh, C. M., Hawkins, E., et al. 2002, MNRAS, 332, 827

Norberg, P., Baugh, C. M., Gaztañaga, E., \& Croton, D. J. 2009, MNRAS, 396, 19

Patej, A., \& Eisenstein, D. 2016, MNRAS, 460, 1310

Peebles, P. J. E. 1980, The Large-scale Structure of the Universe (USA: Princeton University Press)

Pen, U.-L., Lu, T., van Waerbeke, L., \& Mellier, Y. 2003, MNRAS, 346, 994

Planck Collaboration XIII, 2016, A\&A, 594, A13

Prat, J., Sánchez, C., Miquel, R., et al. 2018, MNRAS, 473, 1667

Reyes, R., Mandelbaum, R., Seljak, U., et al. 2010, Nature, 464, 256

Saghiha, H., Simon, P., Schneider, P., \& Hilbert, S. 2017, A\&A, 601, A98
Schmidt, M. 1968, ApJ, 151, 393

Schneider, P. 1998, ApJ, 498, 43

Schneider, A., \& Teyssier, R. 2015, JCAP, 12, 049

Schneider, P., Kochanek, C., \& Wambsganss, J. 2006, Gravitational Lensing: Strong, Weak and Micro, Saas-Fee Advanced Course: Swiss Society for Astrophysics and Astronomy (Springer)

Seljak, U. 2000, MNRAS, 318, 203

Seljak, U., Makarov, A., Mandelbaum, R., et al. 2005, Phys. Rev. D, 71, 043511 Sellentin, E., \& Heavens, A. F. 2016, MNRAS, 456, L132

Sheth, R. K., \& Tormen, G. 1999, MNRAS, 308, 119

Simon, P., \& Hilbert, S. 2018, A\&A, 613, A15

Simon, P., Hetterscheidt, M., Schirmer, M., et al. 2007, A\&A, 461, 861

Simon, P., Erben, T., Schneider, P., et al. 2013, MNRAS, 430, 2476

Simon, P., Saghiha, H., Hilbert, S., et al. 2019, A\&A, 622, A104

Singh, S., Mandelbaum, R., Seljak, U., Slosar, A., \& Vazquez Gonzalez, J. 2017, MNRAS, 471, 3827

Smith, R. E., Peacock, J. A., Jenkins, A., et al. 2003, MNRAS, 341, 1311

Somerville, R. S., Lemson, G., Sigad, Y., et al. 2001, MNRAS, 320, 289

Springel, V., White, S. D. M., Jenkins, A., et al. 2005, Nature, 435, 629

Springel, V., Pakmor, R., Pillepich, A., et al. 2018, MNRAS, 475, 676

Swanson, M. E. C., Tegmark, M., Blanton, M., \& Zehavi, I. 2008, MNRAS, 385, 1635

Takada, M., \& Jain, B. 2002, MNRAS, 337, 875

Takahashi, R., Sato, M., Nishimichi, T., Taruya, A., \& Oguri, M. 2012, ApJ, 761, 152

Takeuchi, T. T., Yoshikawa, K., \& Ishii, T. T. 2000, ApJS, 129, 1

Tegmark, M., \& Peebles, P. J. E. 1998, ApJ, 500, L79

Tegmark, M., \& Bromley, B. C. 1999, ApJ, 518, L69

Tinker, J. L., Weinberg, D. H., Zheng, Z., \& Zehavi, I. 2005, ApJ, 631, 41

Unruh, S., Schneider, P., Hilbert, S., et al. 2020, A\&A, 638, A96

van Daalen, M. P., Henriques, B. M. B., Angulo, R. E., \& White, S. D. M. 2016, MNRAS, 458, 934

van Waerbeke, L. 1998, A\&A, 334, 1

Velander, M., van Uitert, E., Hoekstra, H., et al. 2014, MNRAS, 437, 2111

Vogelsberger, M., Marinacci, F., Torrey, P., \& Puchwein, E. 2020, Nat. Rev. Phys., 2, 42

Weinberg, D. H., Davé, R., Katz, N., \& Hernquist, L. 2004, ApJ, 601, 1

Wild, V., Peacock, J. A., Lahav, O., et al. 2005, MNRAS, 356, 247

Wright, A. H., Driver, S. P., \& Robotham, A. S. G. 2018, MNRAS, 480, 3491

Zehavi, I., Zheng, Z., Weinberg, D. H., et al. 2005, ApJ, 630, 1 


\section{Appendix A: Reconstructed biasing functions}
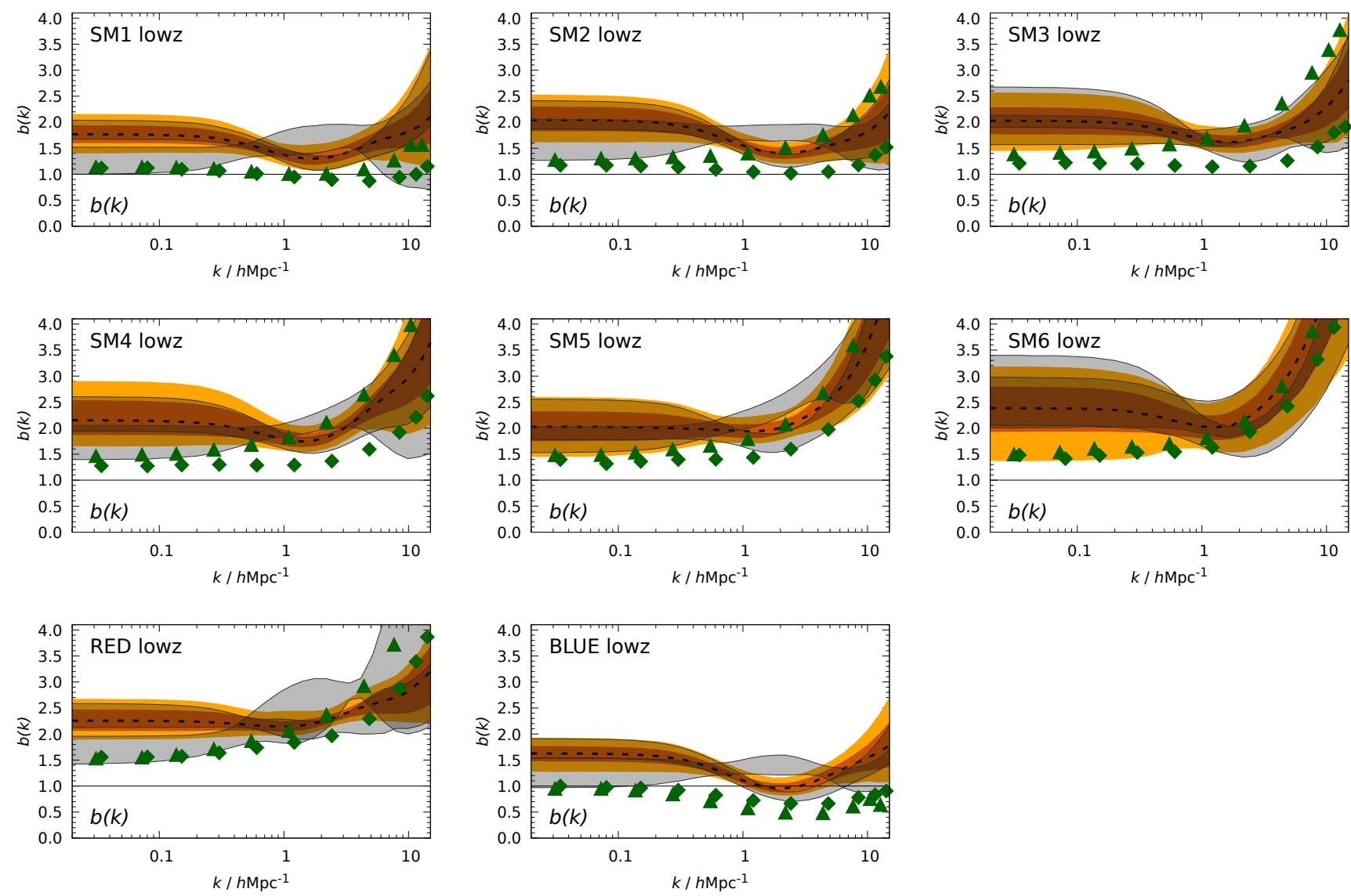

Fig. A.1. Posterior scale-dependent bias $b(k)$ for galaxy samples at redshift $\bar{z} \approx 0.35$. The galaxy sample is indicated in the top left corner. The orange filled regions and the dashed lines show the median, $68 \%$, and $95 \%$ credible region for the CFHTLenS measurement using the full source sample BACK low- $z$. The overlapping transparent grey regions show $68 \%$ regions that are based on the source subsamples $\mathrm{BACKa}$ and $\mathrm{BACKb}$ low- $z$. These noisier measurements at mean source redshifts $\bar{z} \approx 0.86$ and $\bar{z} \approx 1.12$ test the robustness of the measurement. The filled green diamonds and triangles indicate the SAM predictions by H15 and L12, respectively. 

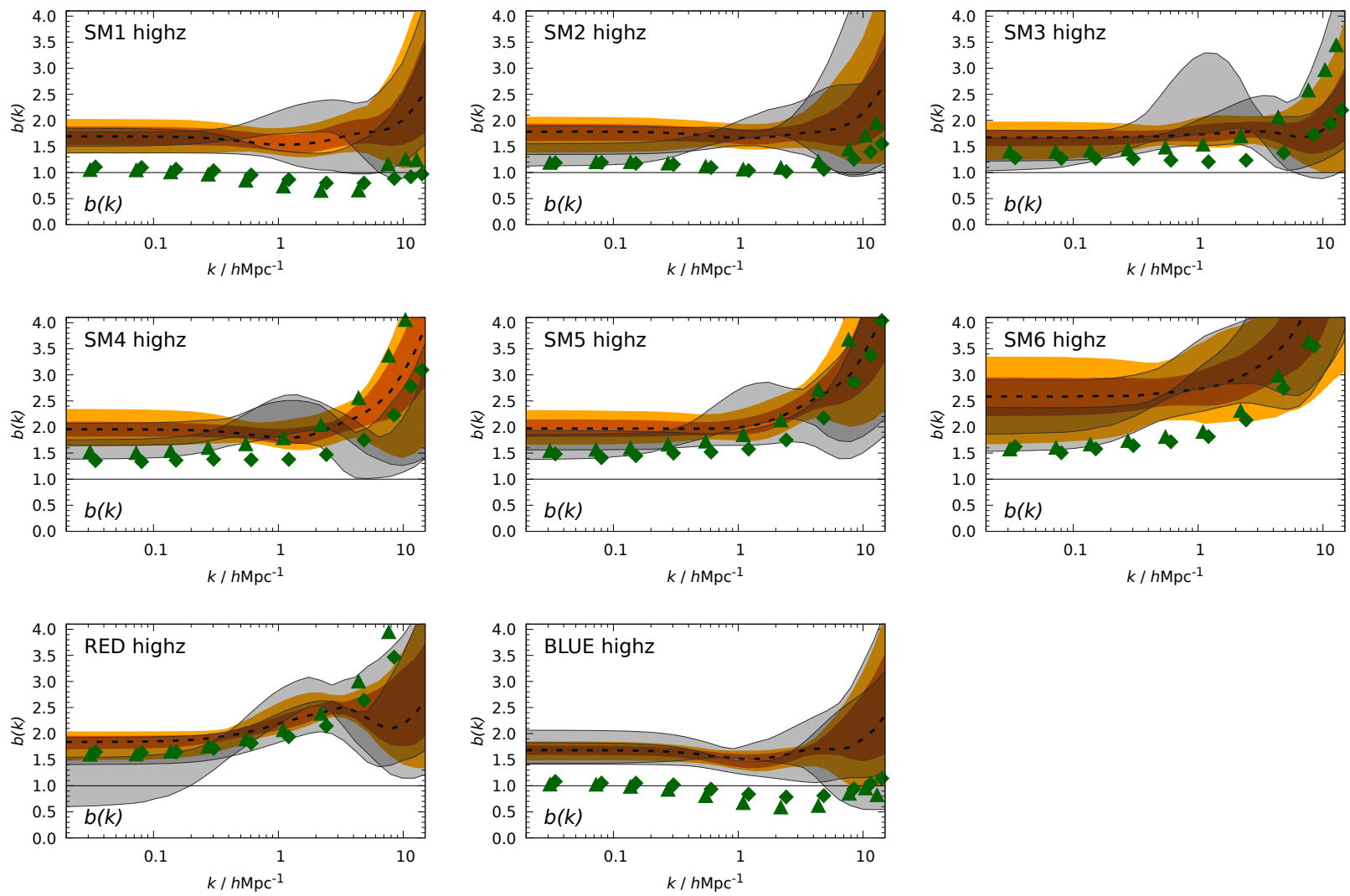

Fig. A.2. As in Fig. A.1, but for the scale-dependent bias $b(k)$ of the high- $z$ galaxy samples $(\bar{z} \approx 0.52)$. The grey regions depict $68 \%$ credible regions, based on the source subsamples BACKa and BACKb high $-z(\bar{z} \approx 0.93,1.14)$. The green data points are SAM predictions.
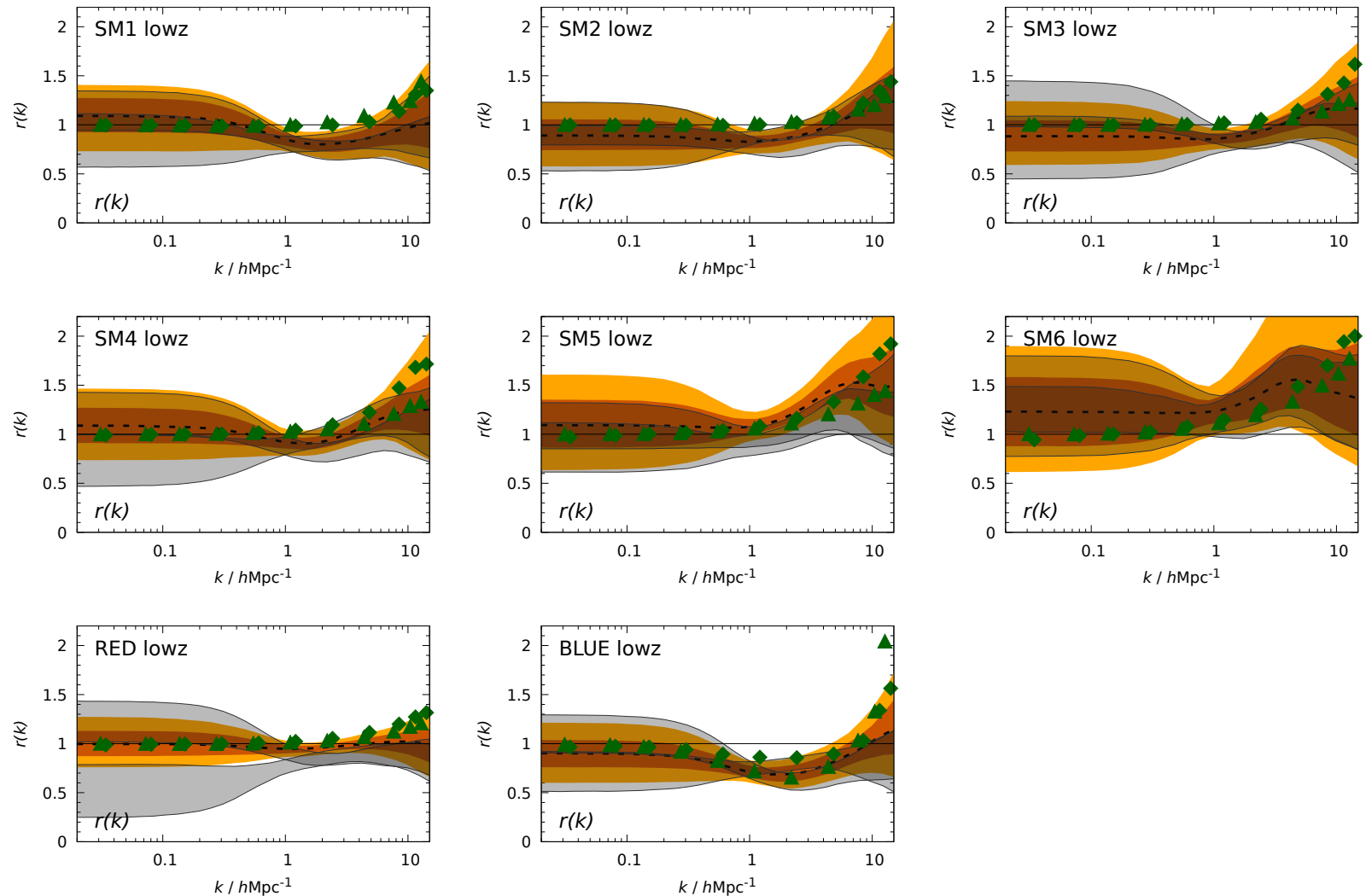

Fig. A.3. As in Fig. A.1, but for the scale-dependent correlation factor $r(k)$ of the low- $z$ galaxy samples $(\bar{z} \approx 0.35)$. The grey $68 \%$ regions are based on two source subsamples. The green data points are SAM predictions. 

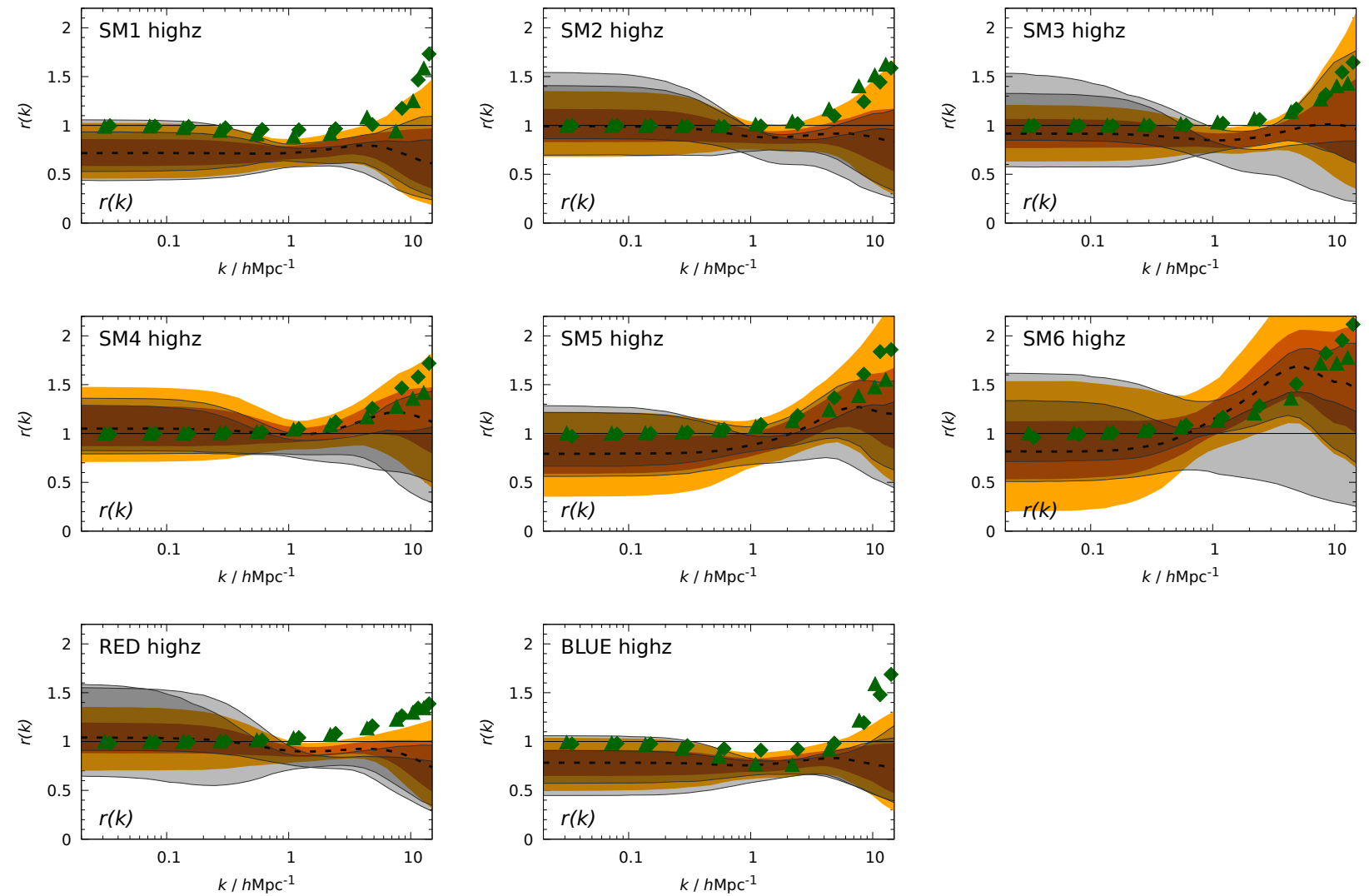

Fig. A.4. As in Fig. A.1, but for the scale-dependent correlation factor $r(k)$ of the high- $z$ samples $(\bar{z} \approx 0.52)$. The grey $68 \%$ regions are based on two source subsamples. The green data points are SAM predictions.

\section{Appendix B: Deconvolution of binned redshift distributions}

To estimate the redshift distribution of our lens galaxies from the binned BPZ posterior distribution we discuss here a maximumlikelihood technique. Generally, the binning of a probability density $p(z) \mathrm{d} z$ into $i=0 \ldots\left(N_{\text {bin }}-1\right)$ bins,

$p_{i}:=\int_{i \Delta z}^{(i+1) \Delta z} \mathrm{~d} z p(z)$,

is not invertible with respect to $p(z)$. By restricting the inversion for $z>0$ to a family of densities of four parameters $\boldsymbol{\Theta}=\left(\bar{z}, \sigma_{z}, s, k\right)$,

$p(z \mid \lambda, \boldsymbol{\Theta})=\lambda \mathrm{e}^{-\frac{\epsilon}{z}} \mathrm{e}^{-\frac{x^{2}}{2}}\left(1+\frac{s}{6} H e_{3}(x)+\frac{k}{24} H e_{4}(x)\right) ; x=\frac{z-\bar{z}}{\sigma_{z}}$,

however, the problem is tractable for our lens samples; we set $p(z \mid \lambda, \boldsymbol{\Theta})=0$ for $z \leq 0$. The normalisation $\lambda$ is defined through $\int_{0}^{\infty} \mathrm{d} z p_{\mathrm{d}}(z \mid \lambda, \boldsymbol{\Theta})=1$, and the functions $\mathrm{He}_{3}(x)=x^{3}-3 x$ and $\mathrm{He}_{4}(x)=x^{4}-6 x^{2}+3$ are Hermite polynomials. This family of models (B.2) is a Gram-Charlier series that approximates the narrow redshift distributions of samples inside photometric redshift bins by a normal distribution and corrections for a skewness or kurtosis. In addition, the exponential function with $\epsilon=10^{-2}$ asserts $p_{\mathrm{d}}(z \mid \lambda, \boldsymbol{\Theta}) \rightarrow 0$ for $z \rightarrow 0$. This correction influences the distribution function only very close to $z=0$. The aim of our technique is to determine the parameters $\Theta$ for given $p_{i}$.

To determine the best-fitting parameters in (B.2) for a given $\mathrm{BPZ}$ posterior, we find the binned distribution

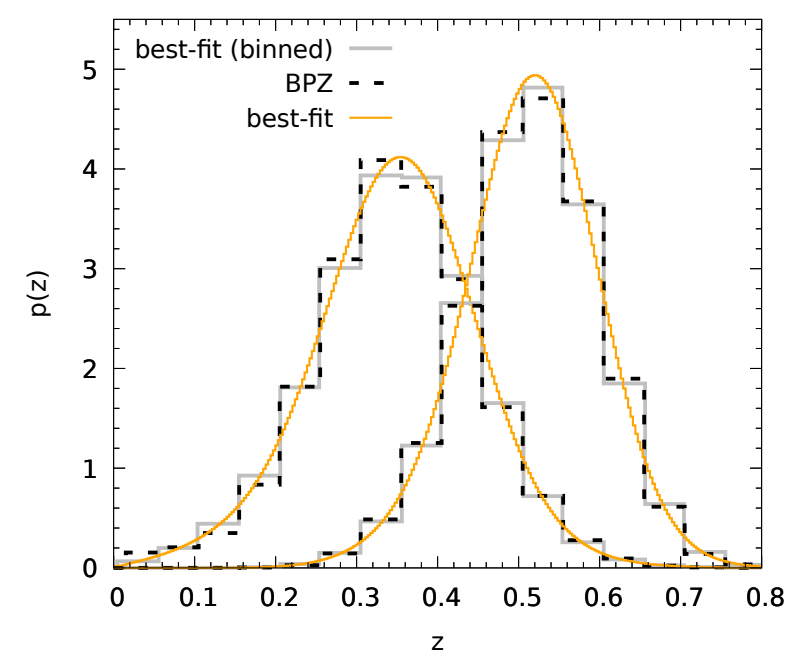

Fig. B.1. Two examples of maximum-likelihood fits of models of $p(z)$ to the combined binned posterior of the BPZ redshift. The left-hand peak is for the low- $z$ bin of lenses SM1 to SM6 combined; the right-hand peak corresponds to the high- $z$ bin. The dashed histograms are the average BPZ posteriors of lenses; the solid histograms are the binned best fits. The smooth curves are the unbinned best fits as used in the analysis.

$\hat{p}_{i}(\lambda, \boldsymbol{\Theta}):=\int_{i \Delta z}^{(i+1) \Delta z} \mathrm{~d} z p(z \mid \lambda, \boldsymbol{\Theta})$

that has the minimal Kullback-Leibler divergence with respect to $p_{i}$, which means finding the $\boldsymbol{\Theta}_{\mathrm{ml}}$ that minimise 


$$
\begin{aligned}
d_{\mathrm{KL}}(\boldsymbol{\Theta}) & =-\sum_{i=0}^{N_{\mathrm{bin}}-1} p_{i} \ln \left(\frac{\hat{p}_{i}(\lambda, \boldsymbol{\Theta})}{p_{i}}\right) \\
& =\text { const }-\sum_{i=0}^{N_{\text {bin }}-1} p_{i} \ln \hat{p}_{i}(\lambda, \boldsymbol{\Theta}) .
\end{aligned}
$$

These values are the maximum-likelihood parameters in the family of model distributions (Knight 1999).

Figure B.1 shows two examples of this fitting procedure for a galaxy sample SM1-SM6 in the two photo- $z$ bins low- $z$ and high$z$. In this figure the dashed histograms are the binned BPZ posteriors $p_{i}$, the solid histograms are the best-fitting binned models $\hat{p}_{i}$, and the solid orange lines are the best-fitting $p(z \mid \lambda, \boldsymbol{\Theta})$ used in the analysis.

\section{Appendix C: $V_{\text {max }}$-estimator for photo- $z$ selected galaxies}

For our modification of the $V_{\max }$-estimator, we consider galaxies that are selected in magnitude $m_{\min } \leq m<m_{\max }$ and from a photo- $z$ interval $z_{\min } \leq z_{\mathrm{ph}}<z_{\max }$. Due to the latter there is a non-negligible probability that galaxies below $z \leq z_{\min }$ or above $z \geq z_{\max }$ are also selected; the selection thus does not clearly define a survey volume. To implement the volume uncertainty in an estimator of the galaxy number density, we assume that we know of every galaxy $i$ the probability density $p_{i}\left(z \mid z_{\mathrm{ph}}\right)$ of its true redshift $z$ given its photometric redshift, and that we know the probability density $p_{i}\left(z_{\mathrm{ph}} \mid z\right)$ of photometric redshifts given the true galaxy redshift. We first study how galaxies are selected.

To define the selection of galaxies, let $p_{i}(z, \boldsymbol{\theta}) \mathrm{d} V=$ $C_{i}(z) S(\boldsymbol{\theta}) \mathrm{d} V$ be the probability that the $i$ th galaxy can be observed inside a comoving volume element $\mathrm{d} V$ in the line-ofsight direction $\boldsymbol{\theta}$ at redshift $z$. The volume element is in a cosmological context

$\mathrm{d} V=f_{K}^{2}(z) \frac{\mathrm{d} \chi(z)}{\mathrm{d} z} \mathrm{~d} \Omega \mathrm{d} z$,

where $\mathrm{d} \Omega$ is an infinitesimal solid angle on the celestial sphere, $f_{K}(z)$ is the comoving angular diameter distance at $z$, and $\chi(z)$ is the comoving distance of a galaxy at $z$. The radial selection $C_{i}(z)$ depends on the survey selection function and the intrinsic properties of the galaxy, and the angular selection $S(\boldsymbol{\theta})$ shall be identical for all galaxies.

Due to the assumed independence of the angular selection on galaxy properties and redshift, all incompleteness effects varying with the position on the sky (e.g. gaps) are accounted for by the effective survey area

$\Omega_{\mathrm{eff}}=\int \mathrm{d} \Omega S(\boldsymbol{\theta})=\int_{-\pi / 2}^{\pi / 2} \int_{0}^{2 \pi} \mathrm{d} \delta \mathrm{d} \alpha \cos \delta S(\boldsymbol{\theta}(\alpha, \delta)) ;$

the right-hand side uses spherical $(\alpha, \delta)$ coordinates on the sky. The effective area of our data, accounting only for gaps $(S=0)$ or no gaps $(S=1)$, is summarised in Table 1 .

For an estimator of the average number density, we assume a homogeneous distribution of selected galaxies throughout the observed volume and, in a preliminary step, imagine that we have only galaxies identical to the $i$ th galaxy; the comoving number density of these galaxies shall be $\bar{n}_{i}$. Then the total detected number $N_{i}$ of galaxies would be

$N_{i}=\int \mathrm{d} V \bar{n}_{i} C_{i}(z) S(\boldsymbol{\theta})=\bar{n}_{i} V_{i}$ with the effective volume

$V_{i}:=\Omega_{\mathrm{eff}} \int_{0}^{\infty} \mathrm{d} z C_{i}(z) f_{K}^{2}(z) \frac{\mathrm{d} \chi(z)}{\mathrm{d} z}$.

In other words, $N_{i} / V_{i}$ is, apart from sampling noise, an estimator of $\bar{n}_{i}$. Since we have only $N_{i}=1$ galaxy $i$ in reality, $1 / V_{i}$ estimates its contribution to the total number density of all $N_{\mathrm{g}}$ galaxies, which is

$\bar{n}_{\mathrm{g}}^{\mathrm{est}}=\sum_{i=1}^{N_{\mathrm{g}}} \frac{1}{V_{i}}$

The individual (radial) selection of the various galaxies is encoded in the $V_{i}$. The original $V_{\max }$-method considers for each $V_{i}$ the redshift interval over which a galaxy, similar to galaxy $i$, would be selected.

This interval is not clearly defined for galaxies with uncertain redshift, however, and we have to modify the original $V_{\max }$ approach by the following two steps. Firstly, for galaxies selected by ( $i^{\prime}$-band) magnitude $m$ and photometric redshift $z_{\mathrm{ph}}$, we calculate the uncertainty in the inferred absolute magnitude $M$ of a galaxy. Let $\left(m_{i}, z_{\mathrm{ph}, i}\right)$ be the apparent magnitude and photometric redshift of galaxy $i$. The luminosity distance $D_{\mathrm{L}}(z)=(1+z) f_{K}(z)$ and therefore the absolute magnitude $M$ of galaxy $i$ is uncertain for its given $z_{\mathrm{ph}, i}$, reflected by a probability $p\left(M \mid m_{i}, z_{\mathrm{ph}, i}\right)$. This $M$ probability is given by marginalising the relation

$M(m, z)=m-5 \log _{10}\left(\frac{D_{\mathrm{L}}(z)}{h^{-1} \mathrm{Mpc}}\right)-25-K(z)-A(z)$

over the $z$-uncertainty $p_{i}\left(z \mid z_{\mathrm{ph}, i}\right)$,

$p\left(M \mid m_{i}, z_{\mathrm{ph}, i}\right)=\int_{0}^{\infty} \mathrm{d} z p_{i}\left(z \mid z_{\mathrm{ph}, i}\right) \delta_{\mathrm{D}}\left(M-M\left(m_{i}, z\right)\right)$,

where $\delta_{\mathrm{D}}(x)$ is the Dirac delta function. The $K$-correction and dust extinction are assumed to be irrelevant here, which means we set $K(z)=A(z)=0$.

Secondly, to account for the $M$ uncertainty in $V_{i}$ we assume an ensemble of galaxies with an intrinsic $M$-distribution (C.7) instead of one particular value $M$ as in the original $V_{\text {max }}$ estimator, and we consider their selection probability when located at different $z$. Placing this ensemble at $z$, we find a joint distribution $p_{i}\left(M, z_{\mathrm{ph}} \mid z\right)=p\left(M \mid m_{i}, z_{\mathrm{ph}, i}\right) \times p_{i}\left(z_{\mathrm{ph}} \mid z\right)$ for $M$ and $z_{\mathrm{ph}}{ }^{8}$. From this ensemble, we select galaxies in intervals of $m$ and $z_{\text {ph }}$ or, more generally, with selection function $0 \leq s\left(m, z_{\mathrm{ph}}\right) \leq 1$. Therefore, the selection probability of an ith-like galaxy at $z$ is

$C_{i}(z)=\int_{0}^{\infty} \mathrm{d} z_{\mathrm{ph}} \mathrm{d} M p_{i}\left(z_{\mathrm{ph}} \mid z\right) p\left(M \mid m_{i}, z_{\mathrm{ph}, i}\right) s\left(m(M, z), z_{\mathrm{ph}}\right)$,

where $m(M, z)$ is $M(m, z)$ inverted with respect to $m$. This allows us to compute $V_{i}$ (Eq. (C.4)) and, if necessary, the density $\bar{n}_{\mathrm{g}}$ (Eq. (C.5)) of all galaxies.

To calculate the integrals in an efficient way we suggest the following numerical method. Using (Eq. (C.8)), the expression

\footnotetext{
8 This is not to be confused with the posterior distribution of $(M, z)$ of the observed galaxy $i$. Rather, we consider similar galaxies with intrinsic $M$ variations at a $z$ different to $z_{i}$.
} 
(C.4) reads

$$
\begin{aligned}
V_{i}=\Omega_{\mathrm{eff}} & \int_{-\infty}^{+\infty} \mathrm{d} M p\left(M \mid m_{i}, z_{\mathrm{ph}, i}\right) \int_{0}^{\infty} \mathrm{d} z \int_{0}^{\infty} \mathrm{d} z_{\mathrm{ph}} \\
& \times f_{K}^{2}(z) \frac{\mathrm{d} \chi(z)}{\mathrm{d} z} p_{i}\left(z_{\mathrm{ph}} \mid z\right) s\left(m(M, z), z_{\mathrm{ph}}\right) .
\end{aligned}
$$

Applying our specific hard selection $s\left(m, z_{\mathrm{ph}}\right) \in\{0,1\}$ with $\left[z_{\min }, z_{\max }\right] \times\left[m_{\min }, m_{\max }\right]$ to the integral limits, we obtain

$$
\begin{aligned}
V_{i}=\Omega_{\mathrm{eff}} & \int_{-\infty}^{+\infty} \mathrm{d} M p\left(M \mid m_{i}, z_{\mathrm{ph}, i}\right) \\
& \times \int_{z\left(M, m_{\min }\right)}^{z\left(M, m_{\max }\right)} \mathrm{d} z f_{K}^{2}(z) \frac{\mathrm{d} \chi(z)}{\mathrm{d} z} \int_{z_{\min }}^{z_{\max }} \mathrm{d} z_{\mathrm{ph}} p_{i}\left(z_{\mathrm{ph}} \mid z\right),
\end{aligned}
$$

where the redshift $z(M, m)$ is implicitly defined by Eq. (C.6). We approximate the integration over $M$ by a Monte Carlo integral that draws a set of $j=1 \ldots n_{\mathrm{mc}}$ values $M_{i j} \sim p\left(M \mid m_{i}, z_{\mathrm{ph}, i}\right)$ (Eq. (C.7)) through $z_{i j} \sim p\left(z \mid z_{\mathrm{ph}, i}\right)$ and $M_{i j}=M\left(m_{i}, z_{i j}\right)$ to obtain

$V_{i} \approx \frac{\Omega_{\mathrm{eff}}}{n_{\mathrm{mc}}} \sum_{j=1}^{n_{\mathrm{mc}}} \int_{z\left(M_{i j}, m_{\min }\right)}^{z\left(M_{i j}, m_{\max }\right)} \mathrm{d} z f_{K}^{2}(z) \frac{\mathrm{d} \chi(z)}{\mathrm{d} z} F_{i}\left[z_{\min }, z_{\max } \mid z\right]$,

where $F_{i}\left[z_{\min }, z_{\max } \mid z\right]$ denotes the probability that galaxy $i$ at redshift $z$ has $z_{\text {ph }} \in\left[z_{\min }, z_{\max }\right]$ :

$F_{i}\left[z_{\min }, z_{\max } \mid z\right]:=\int_{z_{\min }}^{z_{\max }} \mathrm{d} z_{\mathrm{ph}} p_{i}\left(z_{\mathrm{ph}} \mid z\right)$

The approximation in Eq. (C.11) reduces for $n_{\mathrm{mc}} \rightarrow \infty$ and $z \equiv$ $z_{\text {ph }}$ (no photo- $z$ errors) to the observable volume of a galaxy in the original $V_{\max }$-estimator.

To speed up the computation of (C.11), we numerically compute, for a broad range of $z$ values, the volume

$V_{i}[z]:=\Omega_{\mathrm{eff}} \int_{0}^{z} \mathrm{~d} z^{\prime} f_{K}^{2}\left(z^{\prime}\right) \frac{\mathrm{d} \chi\left(z^{\prime}\right)}{\mathrm{d} z^{\prime}} F_{i}\left[z_{\min }, z_{\max } \mid z^{\prime}\right]$,

which we then interpolate to obtain

$V_{i} \approx \frac{1}{n_{\mathrm{mc}}} \sum_{j=1}^{n_{\mathrm{mc}}}\left(V_{i}\left[z\left(M_{i j}, m_{\max }\right)\right]-V_{i}\left[z\left(M_{i j}, m_{\min }\right)\right]\right)$.

In the interpolation, for all galaxies for the photo- $z$ errors, we adopt the (truncated) Gaussian model

$p_{i}\left(z_{\mathrm{ph}} \mid z\right)=\frac{\sqrt{2}}{\sqrt{\pi} \sigma_{z}}\left[1+\operatorname{erf}\left(\frac{z}{\sigma_{z} \sqrt{2}}\right)\right]^{-1} \mathrm{e}^{-\frac{\left(z_{\mathrm{ph}}-z\right)^{2}}{2 \sigma_{z}^{2}}}$

with $\sigma_{z}=(1+z) \delta_{z}$ and $\delta_{z}=0.04$ (Hildebrandt et al. 2012) for $z_{\mathrm{ph}} \geq 0$, and $p_{i}\left(z_{\mathrm{ph}} \mid z\right)=0$ otherwise. The factor in the square brackets with the error function $\operatorname{erf}(x)$ is the normalisation due to the truncation of the normal distribution below $z_{\mathrm{ph}}=0$. With this model of photo- $z$ errors, we can employ the same

$F_{i}\left[z_{\min }, z_{\text {,max }} \mid z\right]=$

$$
\left[1+\operatorname{erf}\left(\frac{z}{\sigma_{z} \sqrt{2}}\right)\right]^{-1} \times\left[\operatorname{erf}\left(\frac{z_{\max }-z}{\sigma_{z} \sqrt{2}}\right)-\operatorname{erf}\left(\frac{z_{\min }-z}{\sigma_{z} \sqrt{2}}\right)\right]
$$

and the same $V_{i}[z]$ for all galaxies in the sample. Moreover, as estimated for $p_{i}\left(z \mid z_{\mathrm{ph}, i}\right)$ and needed for $M_{i j}$, we use the BPZ posterior density of $z$ for the $i$ th galaxy in CFHTLenS.

By means of this analysis we find for our lens low- $z$ (high- $z$ ) samples values of $\bar{n}_{\mathrm{g}}$ that are on average $\sim 3 \%(\sim 1 \%)$ larger than the traditional $V_{\max }$-estimator (assuming $z \equiv z_{\mathrm{ph}}$ ). All results are shown in Fig. 2 as black data points.

\section{Appendix D: Construction of galaxy bias templates}

We use smooth templates of $b(k)$ and $r(k)$ to stabilise the deprojection of the projected galaxy bias. They represent the average scale-dependent galaxy bias of a specifically selected galaxy population with mean redshift $\bar{z}_{\mathrm{d}}$. Details of the derivation can be found in SH18. We give a brief summary here only.

Our templates separate galaxy bias in the two-halo regime from galaxy bias in the one-halo regime. In the two-halo regime, the bias is a stochastic linear bias with two free parameters $b_{\mathrm{ls}}, r_{\mathrm{ls}}: b_{\mathrm{ls}}$ is the large-scale bias factor, and $r_{\mathrm{ls}}$ is the correlation factor.

In the one-halo regime, galaxies populate halos of virial mass $m$. The mean comoving number density of halos at redshift $\bar{z}_{\mathrm{d}}$ is $n(m) \mathrm{d} m$ for the mass interval $m \in[m, m+\mathrm{d} m]$ according to Sheth \& Tormen (1999). The density profile of the dark matter for a halo with mass $m$,

$\rho_{\mathrm{m}}(r, m) \propto r_{\mathrm{vir}}^{-1}\left[1+\frac{r c\left(m, \bar{z}_{\mathrm{d}}\right)}{r_{\mathrm{vir}}}\right]^{-2}$,

follows a Navarro-Frenk-White (NFW) density profile, but is truncated at the virial radius $r_{\text {vir }}$ (Cooray \& Sheth 2002; Navarro et al. 1996); for the concentration parameter $c(m, z)$ at redshift $z=\bar{z}_{\mathrm{d}}$ we adopt the relation in Takada \& Jain (2002). The formalism uses the density profile as a Fourier transform and normalises it by its viral mass:

$\tilde{u}_{\mathrm{m}}(k, m)=\frac{\int_{0}^{r_{\mathrm{vir}}} \mathrm{d} r r k^{-1} \rho_{\mathrm{m}}(r, m) \sin (k r)}{\int_{0}^{r_{\mathrm{vir}}} \mathrm{d} r r^{2} \rho_{\mathrm{m}}(r, m)}$.

For the radial distribution of satellite galaxies inside a halo, we adopt the normalised profile

$\tilde{u}_{\mathrm{g}}(k, m)=\left[\tilde{u}_{\mathrm{m}}(k, m)\right]^{\zeta}$,

where only for $\zeta=1$ satellites have the same radial profile as dark matter. Then we write the biasing functions in the one-halo regime as

$\left[b_{p q}^{1 \mathrm{~h}}(k)\right]^{2}=\frac{\int_{0}^{\infty} \mathrm{d} m n(m) m^{2} \tilde{u}_{\mathrm{g}}^{2 p}(k, m) b(m)^{2}\left(1+\frac{V(m)}{\langle N \mid m\rangle}\right)}{\int_{0}^{\infty} \mathrm{d} m n(m) m^{2} \tilde{u}_{\mathrm{m}}^{2}(k, m)} ;$

$r_{p q}^{1 \mathrm{~h}}(k)=\frac{\int_{0}^{\infty} \mathrm{d} m n(m) m^{2} \tilde{u}_{\mathrm{m}}(k, m) \tilde{u}_{\mathrm{g}}^{q}(k, m) b(m)}{\int_{0}^{\infty} \mathrm{d} m n(m) m^{2} \tilde{u}_{\mathrm{m}}^{2}(k, m)} \frac{1}{b_{p q}^{1 \mathrm{~h}}(k)}$,

for the mean number of galaxies inside halos of mass $m$,

$\langle N \mid m\rangle=\frac{m b(m) \bar{n}_{\mathrm{g}}}{\bar{\rho}_{m}}=: \frac{m b(m)}{m_{\mathrm{piv}} b\left(m_{\mathrm{piv}}\right)}$,

expressed by the mean biasing function $b(m)$ (Cacciato et al. 2012) and a normalisation with the pivotal mass $m_{\text {piv }}$. At $m_{\text {piv }}$ the mean number of galaxies inside a halo is $\left\langle N \mid m_{\text {piv }}\right\rangle=1$. The mean number of galaxy pairs is given by the normalised excess variance $V(m)$ in $\langle N(N-1) \mid m\rangle=\langle N \mid m\rangle[\langle N \mid m\rangle+V(m)]$. If there are no central galaxies, we set $p=q=1$ in the biasing functions, whereas if there are central galaxies in the selected galaxy population for every halo with $N \geq 1$, we use

$p= \begin{cases}1, & \text { for }\langle N(N-1) \mid m\rangle>1 \\ 1 / 2, & \text { otherwise; }\end{cases}$

$q= \begin{cases}1, & \text { for }\langle N \mid m\rangle>1 \\ 0, & \text { otherwise }\end{cases}$ 
The more realistic case is that a galaxy population has central galaxies, but not for all halos that they populate. We achieve this by another parameter $f_{\text {cen }} \in[0,1]$ that mixes halos with central galaxies $p, q$ as in Eq. (D.6) and halos with only satellites ( $p=$ $q=1)$ by the mixing ratio $f_{\text {cen }}$. This mixes the biasing functions in the one-halo regime according to

$\left[b^{1 \mathrm{~h}}(k)\right]^{2}=f_{\text {cen }}\left[b_{p q}^{1 \mathrm{~h}}(k)\right]^{2}+\left(1-f_{\text {cen }}\right)\left[b_{11}^{1 \mathrm{~h}}(k)\right]^{2}$,

$r^{1 \mathrm{~h}}(k)=\frac{b_{p q}^{1 \mathrm{~h}}(k)}{b_{11}^{1 \mathrm{~h}}(k)} \frac{f_{\mathrm{cen}}}{\sqrt{\mathcal{S}_{p q}(k)}} r_{p q}^{1 \mathrm{~h}}(k)+\frac{1-f_{\mathrm{cen}}}{\sqrt{\mathcal{S}_{p q}(k)}} r_{11}^{1 \mathrm{~h}}(k)$,

where we used the shorthand

$\mathcal{S}_{p q}(k):=1-f_{\text {cen }}+f_{\text {cen }}\left(\frac{b_{p q}^{1 \mathrm{~h}}(k)}{b_{11}^{1 \mathrm{~h}}(k)}\right)^{2}$.

The galaxy bias in the two-halo regime is unaffected by the mixing parameter $f_{\text {cen }}$. Unbiased galaxies have $p=q=\zeta=1$, $b(m)=1$, and $V(m)=0$ for all $m$.

For greater flexibility and no strong constraints on the mass dependence of the HOD, both $b(m)$ and $V(m)$ are interpolated between $10^{4}$ and $10^{16} h^{-1} \mathrm{M}_{\odot}$ based on $i=1 \ldots 22$ free interpolation points $b_{i}$ or $V_{i}$ at $m_{i}$,

$b(m)=b_{i}+\frac{b_{i+1}-b_{i}}{\ln m_{i+1}-\ln m_{i}}\left(\ln m-\ln m_{i}\right)$

and

$V(m)=V_{i}+\frac{V_{i+1}-V_{i}}{\ln m_{i+1}-\ln m_{i}}\left(\ln m-\ln m_{i}\right)$

for $m_{i} \leq m \leq m_{i+1}$. The function $b(m)$ has to obey

$\int_{0}^{\infty} \mathrm{d} m n(m) m b(m)=\int_{0}^{\infty} \mathrm{d} m n(m) m$,

which we use to normalise every set of parameters $\left\{b_{i}\right\}$. In total, we have 49 free template parameters $\boldsymbol{\Theta}=$ $\left(\ldots, b_{i}, \ldots, V_{i}, \ldots, \zeta, m_{\text {piv }}, b_{\mathrm{ls}}, r_{\mathrm{ls}}, f_{\text {cen }}\right)$.

Finally, the biasing functions in the one- and two-halo regime are consistently combined into full templates by the relations

$[b(k ; \boldsymbol{\Theta})]^{2}=\left(1-W_{\mathrm{m}}(k)\right)\left[b^{1 \mathrm{~h}}(k)\right]^{2}+W_{\mathrm{m}}(k) b_{\mathrm{ls}}^{2}$

and

$$
\begin{aligned}
r(k ; \boldsymbol{\Theta})= & \sqrt{\left(1-W_{\mathrm{m}}(k)\right)\left(1-W_{\mathrm{g}}(k)\right)} r^{1 \mathrm{~h}}(k) \\
& +\sqrt{W_{\mathrm{m}}(k) W_{\mathrm{g}}(k)} r_{\mathrm{ls}},
\end{aligned}
$$

using the relative weight of the two-halo term in the matter power spectrum $P_{\mathrm{m}}(k ; \chi)$ at $\bar{z}_{\mathrm{d}}$,

$W_{\mathrm{m}}(k)=\frac{P_{\mathrm{m}}^{2 \mathrm{~h}}(k, \chi)}{P_{\mathrm{m}}(k, \chi)}=\left(1+\frac{P_{\mathrm{m}}^{1 \mathrm{~h}}(k, \chi)}{P_{\mathrm{m}}^{2 \mathrm{~h}}(k, \chi)}\right)^{-1}$,

and the expression

$W_{\mathrm{g}}(k):=\left(\frac{b_{\mathrm{ls}}}{b(k ; \boldsymbol{\Theta})}\right)^{2} W_{\mathrm{m}}(k)$.

We estimate $W_{\mathrm{m}}(k)$ by applying the model of the matter power spectrum in Seljak (2000),

$\frac{P_{\mathrm{m}}^{1 \mathrm{~h}}(k, \chi)}{P_{\mathrm{m}}^{2 \mathrm{~h}}(k, \chi)}=\frac{\int_{0}^{\infty} \mathrm{d} m n(m) m^{2} \tilde{u}_{\mathrm{m}}^{2}(k, m)}{P_{\operatorname{lin}}\left(k, \bar{z}_{\mathrm{d}}\right)\left(\int_{0}^{\infty} \mathrm{d} m n(m) m \tilde{u}_{\mathrm{m}}(k, m) b_{\mathrm{h}}\left(m, \bar{z}_{\mathrm{d}}\right)\right)^{2}}$,

where $P_{\text {lin }}(k, z)$ denotes the linear matter power spectrum at redshift $z$ in our fiducial cosmology, and $b_{\mathrm{h}}(\mathrm{m} . \mathrm{z})$ is the bias factor of halos with mass $m$ at redshift $z$, for which we utilise here the model in Tinker et al. (2005).

\section{Appendix E: Template prior}

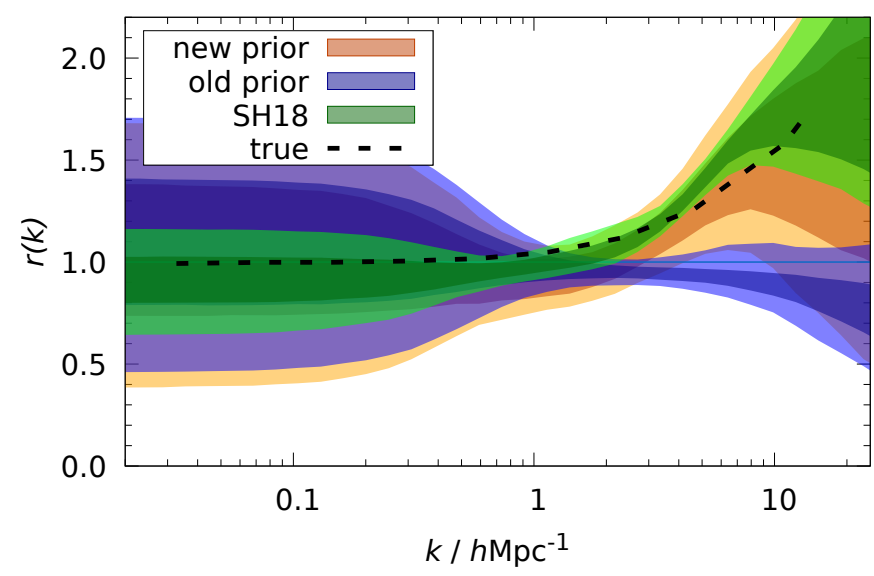

Fig. E.1. Effect of different $b(m)$ prior distributions on the reconstructed $r(k)$ for CFHTLenS-like noisy data (the orange 'new prior' and the blue 'old prior') in comparison to the green SH18-like data with high signalto-noise ratio. The posterior constraints indicate $68 \%$ and $95 \%$ credibility regions. The dashed line is the true $r(k)$ of the H15 semi-analytic galaxies used in this test (SM4 high- $z$ ).

We modify the SH18 prior for the mean biasing function $b(m)$ due to the following reason. The fiducial $\sim 1000 \mathrm{deg}^{2}$ survey in SH18 effectively assumes data with measurement noise roughly a factor of $\sim \sqrt{10}$ smaller than in our CFHTLenS analysis. To make sure that the original setup in SH18 also works for noisier data, we repeated the tests in SH18 with the original synthetic data but using a noise covariance $C$ inflated by a factor of 10 . While the reconstructed $b(k)$ is still consistent with the true galaxy bias in the synthetic data, we found problems for $r(k)$ on scales smaller than $k \gtrsim 3 h \mathrm{Mpc}^{-1}$. This is demonstrated for one example in Fig. E.1. The green contours SH18 show the constraints for $r(k)$ and data with a high signal-to-noise ratio, as in SH18, which is consistent with the true $r(k)$. The blue contours, labelled 'old prior', show the reconstruction with the noisier data and the original $b(m)$ prior in SH18. Clearly, for the old prior and noisier data, the $r(k)$ constraints are too low compared to the true correlation factor (dashed line) on small spatial scales. After a series of tests of our code to possibly identify bugs in the Markov chain Monte Carlo (MCMC) sampler, we found that the original uniform prior within $b(m) \in[0,300]$ is too informative so that it dominates and biases the reconstruction on small scales where our noisier data now makes the likelihood subdominant.

We fixed this problem by using equal probability for different orders of magnitude in the $b(\mathrm{~m})$ prior. As discussed in Sect. 2.9 of Gelman et al. (2003), a uniform prior on a logarithmic scale, which means a prior distribution $p_{\mathrm{b}}\left(b\left(m_{i}\right)\right) \propto b\left(m_{i}\right)^{-1}$, often provides a less informative alternative, which we adopt for each interpolation point $m_{i}$ of $b(m)$ and the orange contours for the 'new prior'. In addition, to restrict the dynamic range of $b(m)$, we reject MCMC proposals for $b(m)$ for which the ratio $\max \{b(m)\} / \min \{b(m)\}$ exceeds $10^{4}$. As a result, the new prior increases the uncertainty on small spatial scales and gives a reconstruction that is now consistent with the correct correlation factor (dashed line). Furthermore, the prior is subdominant for the higher signal-to-noise data in SH18 and both the old and our new prior give similar constraints for $r(k)$ (not shown).

Our new prior for $b(m)$ is easily implemented into the MCMC code by changing from $b(m)$ to $\ln b(m)$ in the 
parametrisation of the galaxy-bias templates. Its practical effect is to prefer small values of $b(m)$ for halo-mass scales $m$ where the likelihood is subdominant (i.e. it suppresses galaxy numbers in these halos). For comparison, the original prior in SH18 prefers a uniform linear-scale scatter around $b(m)=1$ in these cases.

\section{Appendix F: Distribution of systematic errors}

The reconstructed biasing functions rely on parameters for the fiducial cosmology, redshift distributions of lenses and sources, and a model for the intrinsic source alignment (IA). For Fig. F.1, we vary these fiducial parameters for unbiased galaxies in the low $-z$ and high- $z$ redshift bin to simulate the distribution of amplitude errors in $b(k)$ and $r(k)$. As baseline for the error model, we assume the RMS values in Table 6 for (i) the mean and width of the redshift distributions, (ii) the set of fiducial cosmological parameters, (iii) the baryon physics in the non-linear matter power spectrum, and (iv) the amplitude of the IA.
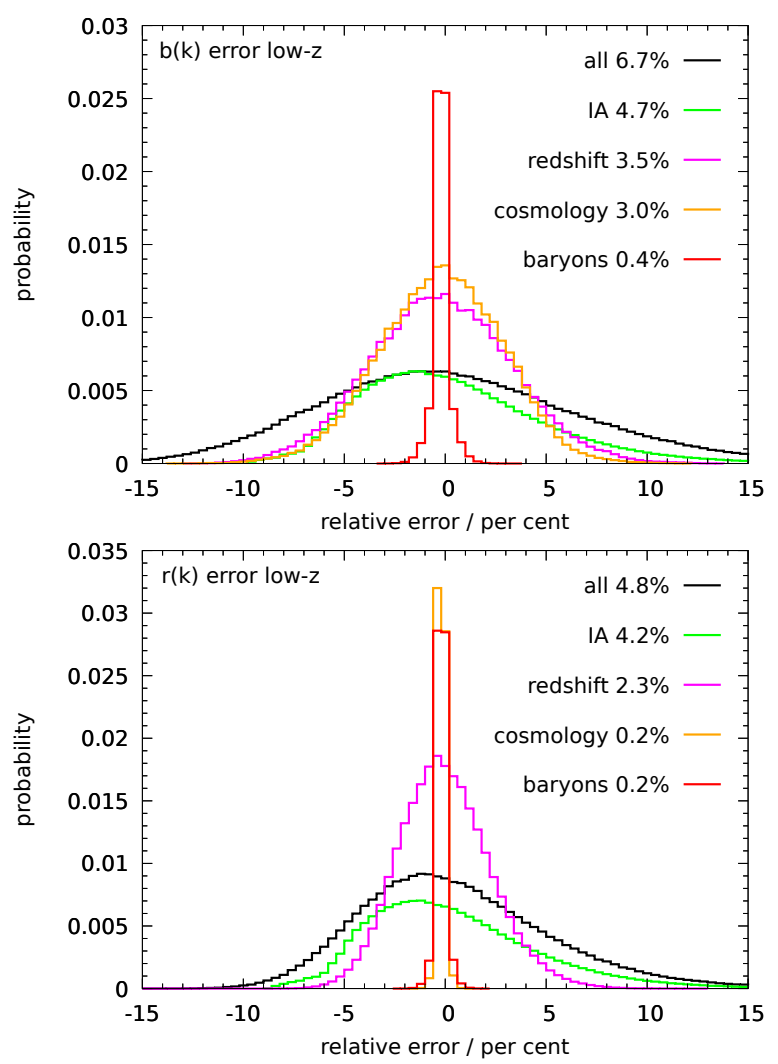

The error distribution for all parameter uncertainties combined is given by the histograms labelled 'all', while the other histograms show the propagated errors with just one parameter variation switched on at a time. The 'all' histograms adopt uncorrelated errors between (i) to (iv) so that the RMS error $\sigma$ of 'all', shown in the figure panels, is the quadratic sum $\sigma^{2}=\sum_{i} \sigma_{i}^{2}$ of the individual RMS values $\sigma_{i}$, which are also indicated in the panels. Realistically, however, we expect the errors to be correlated to some degree. In the worst case, the maximum combined RMS error we can achieve is given by the linear sum $\sigma=\sum_{i} \sigma_{i}$, which provides a conservative upper limit.

In total we find a systematic RMS error for the $b(k)$ amplitude between $6.1 \%$ (uncorrelated) and $11.6 \%$ (conservative), and for $r(k)$ an error between $4.3 \%$ and $6.9 \%$. The errors in the low$z$ bin are about $0.5 \%$ larger than those for high- $z$ due to IA. For $b(k)$, the main contributors to the systematic error are IA, redshift errors, and uncertainties in the fiducial cosmology. For $r(k)$, the cosmology uncertainties are less relevant, while IA and redshift errors are still important.
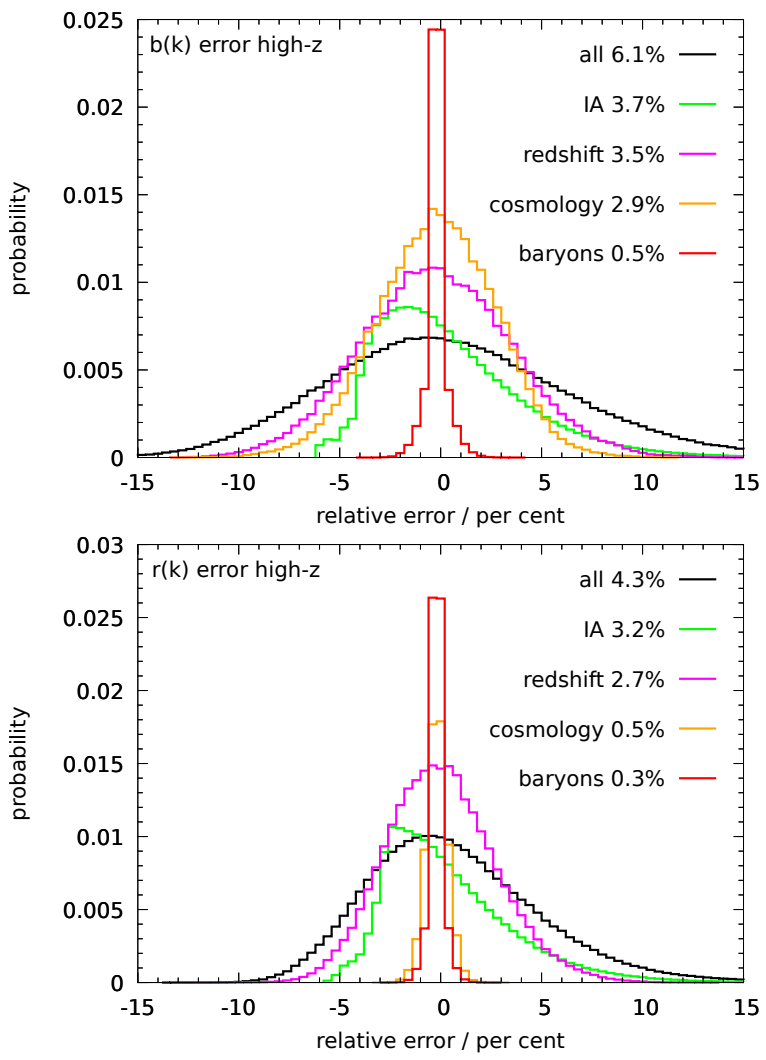

Fig. F.1. Distribution of fractional errors in the reconstructed $b(k)(t o p)$ and $r(k)$ (bottom) for unbiased galaxies. The baseline for systematic errors of parameters is summarised in Table 6 . The left-hand panels show results for the low-z samples; the right-hand panels are for the high- $z$ samples. The lines labelled 'all' (see legend for colours) include errors from all parameters, whereas the other lines show the errors from varying one parameter only. The numbers in each panel's legend give the RMS error. A positive error means that the inferred galaxy bias is systematically too high on all scales. 


\section{Appendix G: Supplemental data on template parameters}

Figures G.1-G.3 summarise our posterior constraints on the template parameters and compare them, where data are available, to the corresponding values in the SAMs. The CFHTLenS errorbars or shaded regions denote $68 \%$ CIs around the posterior median. We note here again that the purpose of the templates is not an accurate modelling of the HOD of galaxies, but the stabilisation of the biasing functions in the deprojection of the ratio statistics. With this in mind, we find the following:

- The CIs of pivotal mass, $m_{\text {piv }}$, shown with filled circles in the left panels of Fig. G.1, span more than one order of magnitude. The median values are almost all above the SAM values (green lines), directly determined from their $\langle N \mid m\rangle$ in the simulation snapshots. This could indicate that the mean halo mass of CFHTLenS galaxies is higher than in the SAMs. However, these values are probably biased high to some degree. This can be seen by the triangle data points, taken from a mock galaxy bias reconstruction in SH18 using H15 galaxies in a $10^{3} \mathrm{deg}^{2}$ CFHTLenSlike mock survey. These data are also somewhat higher than the true values for H15 (solid green lines) in the mock data.

- The fraction $f_{\text {cen }}$ of halos hosting central galaxies in the right panels of Fig. G.1 falls between $20 \%$ to $80 \%$, basically reflecting the Bayesian prior, with a weak increase in the median from SM1 to SM6 in both redshift bins (filled circles). Again, the triangle data points are, for comparison, from the mock analysis in SH18 for H15 data. They are consistent with CFHTLenS but better confined, roughly zooming in to a posterior interval from $70 \%$ to $90 \%$.

- Although the exact shape of $b(m)$ in Fig. G.2 is poorly confined (orange regions), high values of $b(m)$ are typically permitted only within some range of halo mass. This range shifts for low $-z$ (less clear for high- $z$ ) from a few $10^{12}$ to a few $10^{13} h^{-1} \mathrm{M}_{\odot}$ between SM1 and SM6, and the range narrows for larger stellar masses. The BLUE samples suppress $b(m)$ for high halo masses $m \gtrsim 10^{14} h^{-1} \mathrm{M}_{\odot}$, and the RED samples have a strongly varying $b(m)$ over a broad range of halo masses except below a few $10^{11} h^{-1} \mathrm{M}_{\odot}$.

This is qualitatively also seen in the SAMs. The green lines (solid lines: H15; dashed lines: L12) are $b(m)$ for the SAMs, constructed from the $\langle N \mid m\rangle$ at the simulation snapshots by using the definition $b(m)=\Omega_{\mathrm{m}} \bar{\rho}_{\text {crit }}\langle N \mid m\rangle\left(\bar{n}_{\mathrm{g}} m\right)^{-1}$. For SM1-SM6, the
SAM $b(m)$ basically behaves as CFHTLenS: $b(m)$; it is longtailed and peaked around a characteristic halo mass with relatively high galaxy numbers per halo mass, and the peak moves to higher masses for higher stellar mass. Crucially, the SAMs are offset with respect to the CFHTLenS peaks; in other words CFHTLenS galaxies of the same stellar mass seemingly prefer to populate halos of higher mass. However, this tendency towards halos of higher mass is at least partly an artefact of the crude modelling in the templates because the peak position in $b(m)$ for SM5-SM6 low- $z$ and high- $z$ in the analysis by SH18 with H15based mock data (their Fig. D.1) is similarly offset with respect to the true $b(m)$ of $\mathrm{H} 15$.

The offset might also be present for the BLUE and RED samples, but this is far less clear here. As is true for for CFHTLenS, the SAM BLUE galaxies prefer to populate low-mass halos (they are preferentially field galaxies), while RED galaxies prefer halos of higher mass (many red galaxies are satellites).

- The normalised excess variance $V(m)$, grey shaded regions in Fig. G.3, is consistent with a Poisson variance of galaxy numbers inside halos for all galaxy samples $(V(m)=0)$ or possibly a sub-Poisson variance around $10^{13}$ to $10^{14} h^{-1} \mathrm{M}_{\odot}$; SM6 high- $z$ is a prominent example. We note that for $m \lesssim 10^{12} h^{-1} \mathrm{M}_{\odot}$ we have $\langle N \mid m\rangle \ll 1$, and thus a lower limit of $V(m) \geq-\langle N \mid m\rangle \approx 0$ for all samples, which is visible in the plots. The SH18 constraints (68\% CIs) with the H15-based mock data are similar to the CFHTLenS results (green dashed lines).

The green lines (H15: solid; L12: dashed) are the corresponding trends for $V(m)$ in the SAM galaxy samples. They also indicate a sub-Poisson variance around $10^{12} h^{-1} \mathrm{M}_{\odot}$, but with an offset relative to CFHTLenS that is similar to the offset seen for $b(m)$. At higher halo mass of $m \gtrsim 10^{14} h^{-1} \mathrm{M}_{\odot}$ and for the small stellar masses SM1-SM4, H15 shows a super-Poisson variance $(V(m)>0)$ that is not visible in L12. This might be related to the different SAM physics of satellites inside galaxy clusters. The SAM statistics notably becomes noisy for halo masses above a few $10^{14} h^{-1} \mathrm{M}_{\odot}$ where the simulation box quickly runs out of halos.

- There is no evidence that supports a distribution of satellites different to that of matter inside halos for all stellar mass samples $(\zeta \approx 1)$, although the median $\zeta$ tends to decrease for increasing stellar mass. Typical values are $\zeta=1.28_{-0.45}^{+0.44}$ $\left(1.28_{-0.39}^{+0.39}\right)$ for SM1 and $\zeta=0.98_{-0.48}^{+0.56}\left(1.16_{-0.49}^{+0.50}\right)$ for SM6 in the low- $z$ (high- $z$ ) samples. 

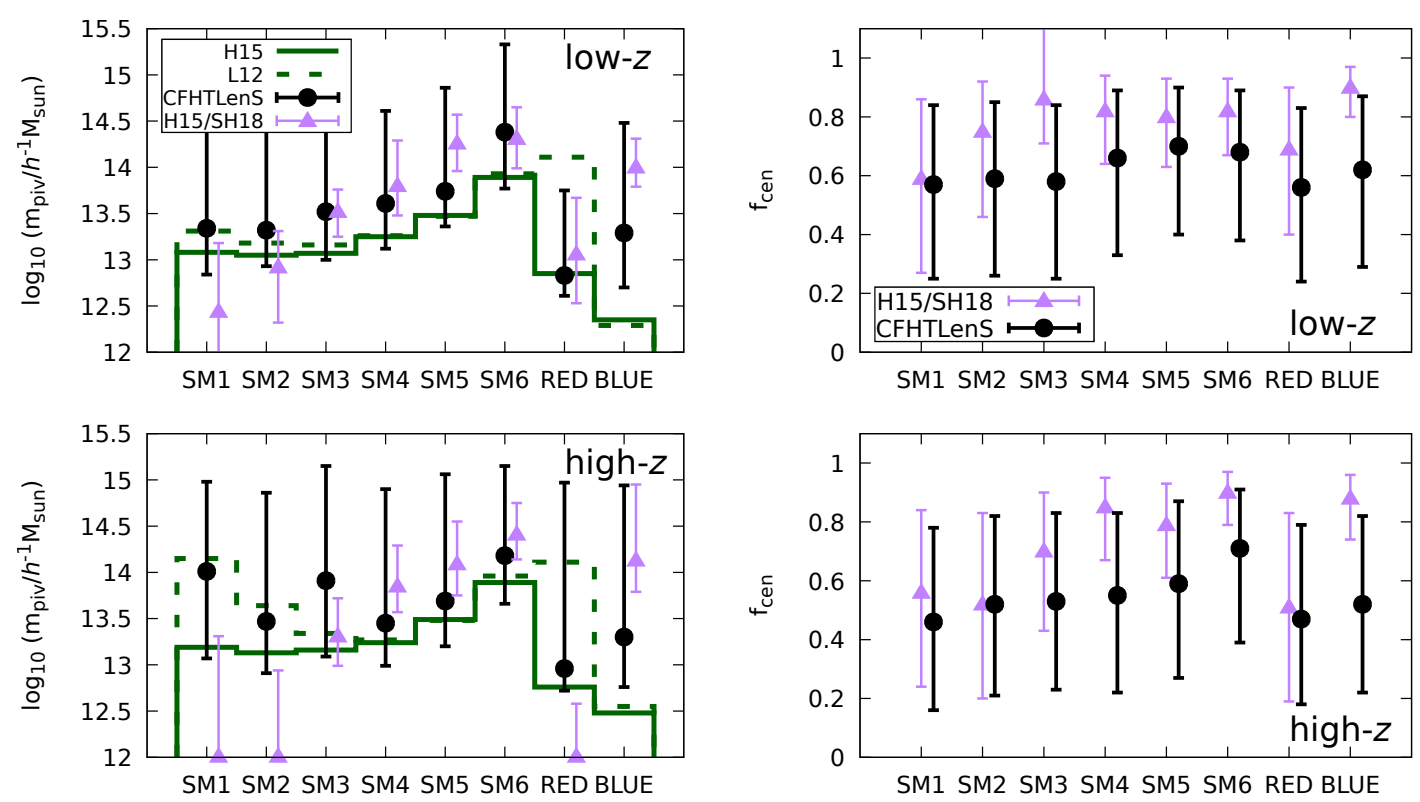

Fig. G.1. Posterior constraints of $m_{\text {piv }}$ (left panels) and $f_{\text {cen }}$ (right panels) with 68\% CIs as median and error bars for all galaxy samples indicated on the $x$-axes; top panels are for low- $z$ samples and bottom panels for high- $z$ samples. The circles are CFHTLenS measurements, and the triangles are measurements from SH18 in a $10^{3} \mathrm{deg}^{2}$ mock survey with $\mathrm{H} 15$ galaxies and galaxy selections as in CFHTLenS. The green lines show $m_{\text {piv }}$ in H15 (solid) and L12 (dotted) directly from the simulation snapshots.
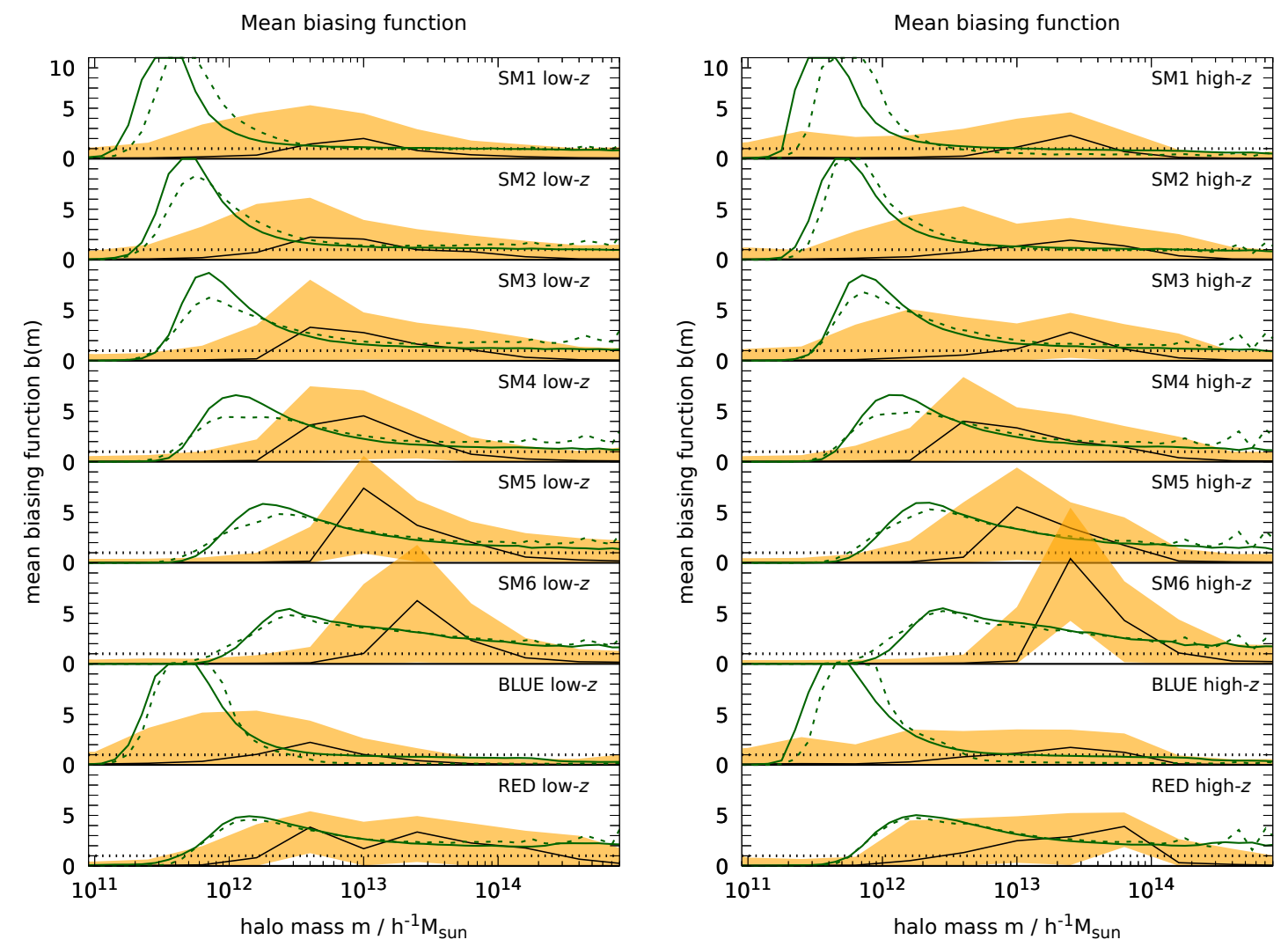

Fig. G.2. Posterior density of the mean biasing function $b(m)=\langle N \mid m\rangle \bar{\rho}_{\mathrm{m}}\left(\bar{n}_{\mathrm{g}} m\right)^{-1}$ as function of halo mass $m$. The low- $z$ samples are in the $l e f t$ panel, the high-z samples in the right panel. The dotted lines indicate $b(m)=1$ for each sample. The shaded regions are the $68 \%$ CIs around the median (solid lines). The green lines show for comparison the $b(m)$ in the SAMs (solid line: H15; dotted lines: L12) directly from the $\langle N \mid m\rangle$ in the simulation snapshots. 


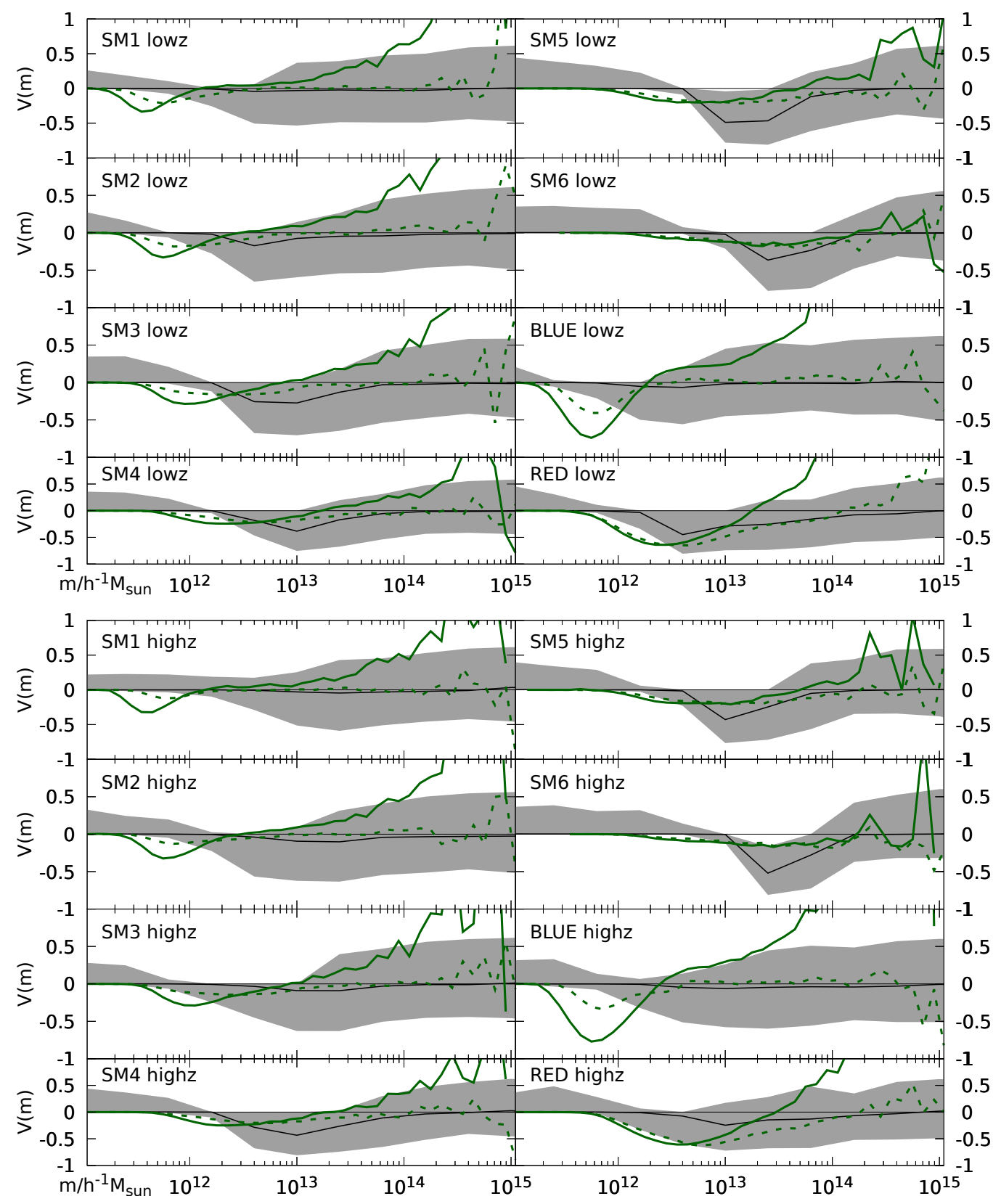

Fig. G.3. Posterior density of the normalised excess variance $V(m)=\left[\langle N(N-1) \mid m\rangle-\langle N \mid m\rangle^{2}\right]\langle N \mid m\rangle^{-1}$ (y-axis) as a function of halo mass $m / h^{-1} \mathbf{M}_{\odot}$ ( $x$-axis) for the low- $z$ galaxy samples (top) and high- $z$ samples (bottom). The shaded regions are the 68\% CIs around the median (black solid lines). The green lines show $V(m)$ for $\mathrm{H} 15$ (solid) and L12 (dashed) directly from the $\langle N(N-1) \mid m\rangle$ in the simulation snapshots. 